\author{
Supporting Information \\ for the \\ Article Entitled
}

\title{
Enantioselective Living Coordinative Chain Transfer Polymerization: Production of Optically Active End-Group-Functionalized (+)- or (-)- Poly(methylene-1,3- cyclopentane) via a Homochiral $C_{1}$-Symmetric Caproamidinate Hafnium Initiator
}

\author{
Authored by \\ Mark A. Wallace, Peter Y. Zavalij, and Lawrence R. Sita* \\ Department of Chemistry and Biochemistry \\ University of Maryland, College Park, Maryland 20742, USA \\ *corresponding author email: Isita@umd.edu
}

Details for the synthesis and isolation of $\left(\boldsymbol{S}_{\mathrm{C}}, \boldsymbol{S}_{H f}\right)-\mathbf{1}$ and $\left(\boldsymbol{R}_{\mathrm{C}}, \boldsymbol{R}_{H f}\right)-\mathbf{1}$. Details for the polymerization and isolation of (+)- and (-)-poly(methylene-1,3-cyclopentane) and poly-1hexene. Supporting NMR Spectra and GPC traces. Crystallographic information regarding $\mathrm{X}$-ray analyses, including tables of bond lengths and angles for $\left(\boldsymbol{S}_{\mathrm{C}}, \boldsymbol{S}_{\boldsymbol{H} f}\right)-\mathbf{1}$ and $\left(R_{\mathrm{C}}, R_{H f}\right)-1$. 


\section{Experimental Details}

\section{General Considerations}

All manipulations with air and moisture sensitive materials were carried out under $\mathrm{N}_{2}$ using standard Schlenk or glovebox techniques. $\mathrm{Et}_{2} \mathrm{O}$ was dried over $\mathrm{Na} /$ benzophenone and distilled under $\mathrm{N}_{2}$ prior to use. Toluene (ReagentPlus, $99 \%$ ) was dried and deoxygenated by passage over activated alumina and activated copper catalyst (GetterMax 135) and collected under $\mathrm{N}_{2}$ prior to use. Chlorobenzene (ReagentPlus, 99\%) was dried over calcium hydride, refluxed at $130{ }^{\circ} \mathrm{C}$ for three days, and distilled under $\mathrm{N}_{2}$ prior to use. Hexane was dried over lithium aluminum hydride, refluxed at $70^{\circ} \mathrm{C}$ for three days, and distilled under $\mathrm{N}_{2}$ prior to use. Celite was oven dried ( $150^{\circ} \mathrm{C}$ for seven days) before use in glovebox. Cooling was performed in the internal freezer of the glovebox maintained at $-20{ }^{\circ} \mathrm{C}$. Toluene- $d_{8}$ was purchased from Cambridge Isotopes, dried over $\mathrm{Na} / \mathrm{K}$ alloy and isolated by vacuum-transfer prior to use. Chloroform- $d_{1}$ was purchased from Cambridge Isotopes and used as received. 1,5-hexadiene, and 1-hexene were purchased from TCl Chemicals, dried over $\mathrm{Na} / \mathrm{K}$ alloy and isolated by vacuum-transfer prior to use. $\left[\mathrm{PhNMe}_{2} \mathrm{H}\right]\left[\mathrm{B}\left(\mathrm{C}_{6} \mathrm{~F}_{5}\right)_{4}\right]$ was purchased from Boulder Scientific and used as received. (-) (S)-1-phenylethylamine $(98 \%)$ and $(+)-(R)-1$-phenylethylamine $(\geq 99 \%)$ were purchased from Sigma-Aldrich and used as received. $\mathrm{Cp}^{*} \mathrm{HfMe}_{3},{ }^{1} \quad(+)-\mathrm{N}-(\mathrm{R})-1$-phenylethyliminocaprolactam, ${ }^{2}$ and (-)-N-(S)-1-phenylethylcaprolactam ${ }^{2}$ were synthesized in excellent yields according to published procedures.

\section{Characterization}

Gel Permeation Chromatography (GPC) was used to obtain molecular weight (Mn and $\mathrm{Mw}$ ) and polydispersity index (PDI) of polymers using Malvern GPCMax equipped with three columns (two Shodex HT-803 and one Shodex HT-804) in a column oven and differential refractometer (maintained at $40^{\circ} \mathrm{C}$ ). Tetrahydrofuran (HPLC Grade) was used as the eluent with a flow rate of $1 \mathrm{~mL} / \mathrm{min}$. Polystyrene standards (from Polymer Laboratories Inc., $580 \mathrm{Da}-3,150 \mathrm{kDa}$ ) were used for calibration. For GPC sample preparation, $10 \mathrm{mg}$ of dry polymer sample was dissolved in $1 \mathrm{~g}$ of THF (HPLC Grade) then filtered through a $0.2 \mu \mathrm{m}$ Nylon Target2 filter.

Nuclear Magnetic Resonance (NMR) Spectroscopy for ${ }^{1} \mathrm{H}$ and ${ }^{13} \mathrm{C}$ nuclei was carried out with a Bruker AV 400 and Bruker DRX $500 \mathrm{MHz}$ spectrometers. Chloroform- $d_{1}$ was used as the solvent for polymer samples. Toluene- $d_{8}$ was used for $\left(\boldsymbol{S}_{\mathrm{c}}, \boldsymbol{S}_{H f}\right)-\mathbf{1}$ and $\left(\boldsymbol{R}_{\mathrm{c}}, \boldsymbol{R}_{\boldsymbol{H}}\right) \mathbf{- 1}$. Spectra were referenced to tetramethylsilane using residual ${ }^{1} \mathrm{H}$ and ${ }^{13} \mathrm{C}$ chemical shifts of the deuterated solvents. 
Optical rotation measurements were collected on a JASCO P1020 Polarimeter using a $589 \mathrm{~nm}$ Sodium Lamp. Chloroform was used for all polymers solutions and toluene was used for $\left(\boldsymbol{S}_{\mathrm{C}}, \boldsymbol{S}_{H f}\right)-1$ and $\left(\boldsymbol{R}_{\mathrm{C}}, \boldsymbol{R}_{H f}\right)-1$.

\section{Synthesis}

Cp*Hf[(N-(-)-(S)-1-phenylethyl)(Nimcap $N)](M e)_{2}\left(\left(S_{C}, S_{H f}\right)-1\right)$. In a $100 \mathrm{~mL}$ Schlenk flask, $0.274 \mathrm{~g}$ of $\mathrm{Cp}^{*} \mathrm{HfMe}_{3}(0.764 \mathrm{mmol})$ in $14 \mathrm{~mL}$ of $\mathrm{Et}_{2} \mathrm{O}$ was chilled to $-20{ }^{\circ} \mathrm{C}$. In a $25 \mathrm{~mL}$ glass vial, $0.157 \mathrm{~g}$ of (S)-IIla $(0.726 \mathrm{mmol})$ in $15 \mathrm{~mL}$ of $\mathrm{Et}_{2} \mathrm{O}$ was chilled to $-20{ }^{\circ} \mathrm{C}$. The solution of (S)-IIla was added dropwise to the stirring solution of $\mathrm{Cp}^{*} \mathrm{HfMe}_{3}$ over 10 minutes. After the addition of (S)-IIla, the reaction mixture was stirred at room temperature for 17.5 hours. Solvent was removed in vacuo, and the residue was dissolved in hexane and filtered through a glass frit with a small pad of celite. Solvent was removed in vacuo, and the white solid was dissolved in minimal hexane and recrystallized at $-20^{\circ} \mathrm{C}$ for three days yielding a white crystalline solid $(0.350 \mathrm{~g}, 86 \%) .{ }^{1} \mathrm{H}$ NMR $\left(400 \mathrm{MHz}\right.$, Tol- $\left.d_{8}, 25^{\circ} \mathrm{C}\right)$ : $\delta(\mathrm{ppm}) 7.35(2 \mathrm{H}, d, \mathrm{~J}=7.89 \mathrm{~Hz}, \mathrm{Ar}), 7.17(2 \mathrm{H}, t, \mathrm{~J}=7.55 \mathrm{~Hz}, \mathrm{Ar}), 7.02(1 \mathrm{H}, t, \mathrm{~J}=7.21$ $\mathrm{Hz}, \mathrm{Ar}), 4.51\left(1 \mathrm{H}, q, \mathrm{~J}=6.97 \mathrm{~Hz}, \mathrm{PhC}(H) \mathrm{CH}_{3}\right), 2.96\left(2 \mathrm{H}, \mathrm{m}, \mathrm{NCH}_{2}\right) 2.02(15 \mathrm{H}, s$, $\left.\mathrm{C}_{5}\left(\mathrm{CH}_{3}\right)_{5}\right), 1.98(1 \mathrm{H}, \mathrm{m}, \mathrm{CHH}), 1.81(1 \mathrm{H}, \mathrm{m}, \mathrm{CHH}), 1.42\left(3 \mathrm{H}, d, \mathrm{~J}=7.02 \mathrm{~Hz}, \mathrm{CH}\left(\mathrm{CH}_{3}\right)\right.$, 0.82-1.41 (6H, br, $\left.\left(\mathrm{CH}_{2}\right)_{3}\right),-0.01\left(3 \mathrm{H}, \mathrm{s}, \mathrm{M}\left(\mathrm{CH}_{3}\right)\right),-0.02\left(3 \mathrm{H}, \mathrm{s}, \mathrm{M}\left(\mathrm{CH}_{3}\right) .{ }^{13} \mathrm{C}\left\{{ }^{1} \mathrm{H}\right\}(100 \mathrm{MHz}\right.$, Tol-d $\left.d_{8}, 25^{\circ} \mathrm{C}\right): \delta(\mathrm{ppm}) 181.2(\mathrm{NC} \mathrm{N}), 148.4\left(\mathrm{C}_{6} \mathrm{H}_{5}\right), 128.4\left(\mathrm{C}_{6} \mathrm{H}_{5}\right), 126.7\left(\mathrm{C}_{6} \mathrm{H}_{5}\right), 126.3$ $\left(\mathrm{C}_{6} \mathrm{H}_{5}\right), 118.5\left(\mathrm{C}_{5}\left(\mathrm{CH}_{3}\right)_{5}\right), 55.6\left(\mathrm{NCH}\left(\mathrm{C}_{6} \mathrm{H}_{5}\right), 52.1,\left(\mathrm{MCH}_{3}\right), 52.0,\left(\mathrm{MCH}_{3}\right), 47.6\left(\mathrm{CH}\left(\mathrm{CH}_{3}\right)\right)\right.$, $31.1\left(\mathrm{CH}_{2}\right), 30.7\left(\mathrm{CH}_{2}\right), 30.1\left(\mathrm{CH}_{2}\right), 24.2\left(\mathrm{CH}_{2}\right), 23.7\left(\mathrm{CH}_{2}\right), 11.4\left(\mathrm{C}_{5}\left(\mathrm{CH}_{3}\right)_{5}\right.$. Anal. Calcd for $\mathrm{C}_{26} \mathrm{H}_{40} \mathrm{~N}_{2} \mathrm{Hf}$ : \%C 55.85, \%H 7.21, \%N 5.01. Found \%C, 54.78, \%H 7.49, \%N 4.87. $[\alpha]_{D}^{26}+31.80^{\circ}$ (c 1.00, Toluene).

Cp*Hf[(N-(+)-(R)-1-phenylethyl)(N ${ }^{\text {imcap }}$ )] $(\mathbf{M e})_{2}\left(\left(\boldsymbol{R}_{\mathrm{C}}, \boldsymbol{R}_{H f}\right)-1\right)$. In a $100 \mathrm{~mL}$ Schlenk flask, $0.335 \mathrm{~g}$ of $\mathrm{Cp}^{*} \mathrm{HfMe}_{3}(0.933 \mathrm{mmol})$ in $15 \mathrm{~mL}$ of $\mathrm{Et}_{2} \mathrm{O}$ was chilled to $-20^{\circ} \mathrm{C}$. In a separate $25 \mathrm{~mL}$ glass vial, $0.192 \mathrm{~g}$ of (R)-IIla $(0.886 \mathrm{mmol})$ in $15 \mathrm{~mL}$ of $\mathrm{Et}_{2} \mathrm{O}$ was chilled to $-20^{\circ} \mathrm{C}$. The solution of (R)-IIla was added dropwise to the stirring solution of $\mathrm{Cp}^{*} \mathrm{HfMe}_{3}$ over ten minutes. After the addition, the reaction mixture was stirred at room temperature for 18 hours. Solvent was removed in vacuo, and the residue was dissolved in hexane and filtered through a glass frit with a small pad of celite. Solvent was removed in vacuo, and the white solid was dissolved in minimal hexane and recrystallized at $-20^{\circ} \mathrm{C}$ for three days yielding a white crystalline solid $(0.411 \mathrm{~g}, 83 \%) .{ }^{1} \mathrm{H}$ NMR $\left(400 \mathrm{MHz}\right.$, Tol- $\left.d_{8}, 2{ }^{\circ} \mathrm{C}\right): \delta$ (ppm) $7.35(2 \mathrm{H}, d, \mathrm{~J}=7.89 \mathrm{~Hz}, \mathrm{Ar}), 7.17(2 \mathrm{H}, t, \mathrm{~J}=7.55 \mathrm{~Hz}, \mathrm{Ar}), 7.02(1 \mathrm{H}, t, \mathrm{~J}=7.21 \mathrm{~Hz}$, $\mathrm{Ar}), 4.51\left(1 \mathrm{H}, q, \mathrm{~J}=6.97 \mathrm{~Hz}, \mathrm{PhC}(H) \mathrm{CH}_{3}\right), 2.96\left(2 \mathrm{H}, \mathrm{m}, \mathrm{NCH}_{2}\right) 2.02\left(15 \mathrm{H}, \mathrm{s}, \mathrm{C}_{5}\left(\mathrm{CH}_{3}\right)_{5}\right)$, $1.98(1 \mathrm{H}, \mathrm{m}, \mathrm{CHH}), 1.81(1 \mathrm{H}, \mathrm{m}, \mathrm{CHH}), 1.42\left(3 \mathrm{H}, d, \mathrm{~J}=7.02 \mathrm{~Hz}, \mathrm{CH}\left(\mathrm{CH}_{3}\right), 0.82-1.41(6 \mathrm{H}\right.$, br, $\left.\left(\mathrm{CH}_{2}\right)_{3}\right),-0.01\left(3 \mathrm{H}, \mathrm{s}, \mathrm{M}\left(\mathrm{CH}_{3}\right)\right),-0.02\left(3 \mathrm{H}, \mathrm{s}, \mathrm{M}\left(\mathrm{CH}_{3}\right) .{ }^{13} \mathrm{C}\left\{{ }^{1} \mathrm{H}\right\}\left(100 \mathrm{MHz}, \mathrm{Tol}-d_{8}, 25\right.\right.$ $\left.{ }^{\circ} \mathrm{C}\right): \delta(\mathrm{ppm}) 181.2(\mathrm{NC} \mathrm{N}), 148.4\left(\mathrm{C}_{6} \mathrm{H}_{5}\right), 128.4\left(\mathrm{C}_{6} \mathrm{H}_{5}\right), 126.7\left(\mathrm{C}_{6} \mathrm{H}_{5}\right), 126.31\left(\mathrm{C}_{6} \mathrm{H}_{5}\right)$, $118.57\left(\mathrm{C}_{5}\left(\mathrm{CH}_{3}\right)_{5}\right), 55.67\left(\mathrm{NC} \mathrm{H}\left(\mathrm{C}_{6} \mathrm{H}_{5}\right), 52.13,\left(\mathrm{MCH}_{3}\right)\right.$, 52.05, $\left(\mathrm{MCH}_{3}\right), 47.6\left(\mathrm{CH}\left(\mathrm{CH}_{3}\right)\right)$, $31.1\left(\mathrm{CH}_{2}\right), 30.7\left(\mathrm{CH}_{2}\right), 30.1\left(\mathrm{CH}_{2}\right), 24.2\left(\mathrm{CH}_{2}\right), 23.7\left(\mathrm{CH}_{2}\right), 11.4\left(\mathrm{C}_{5}\left(\mathrm{CH}_{3}\right)_{5}\right.$. Anal. Calcd 
for $\mathrm{C}_{26} \mathrm{H}_{40} \mathrm{~N}_{2} \mathrm{Hf}$ : \%C 55.85, \%H 7.21, \%N 5.01. Found \%C 54.85, \%H 7.30, \%N 5.03. $[\alpha]_{D}^{26}-31.18^{\circ}(c 1.00$, Toluene).

\section{Polymerization Procedures}

General Procedure for LCP of 1,5-hexadiene and 1-Hexene. In a $25 \mathrm{~mL}$ round bottom, to a stirring $5 \mathrm{~mL}$ solution of $66.0 \mathrm{mg}(0.083 \mathrm{mmol})$ of $\left[\mathrm{PhNMe}_{2} \mathrm{H}\right]\left[\mathrm{B}\left(\mathrm{C}_{6} \mathrm{~F}_{5}\right)_{4}\right]$ in chlorobenzene chilled to $-5^{\circ} \mathrm{C}$ was added $42.0 \mathrm{mg}(0.075 \mathrm{mmol})$ of $\left(\boldsymbol{S}_{\mathrm{c}}, \boldsymbol{S}_{H f}\right)-1 \mathrm{in} 1 \mathrm{~mL}$ of chlorobenzene pre-chilled to $-5^{\circ} \mathrm{C}$. The mixture was stirred and allowed to equilibrate for 20 minutes turning a pale-yellow color. To the stirring solution $0.924 \mathrm{~g}$ of 1,5 -hexadiene $(11.3 \mathrm{mmol})$ was added all at once, and the reaction mixture was stirred for 21 hours at $5{ }^{\circ} \mathrm{C}$ before precipitating into $500 \mathrm{~mL}$ of acidic methanol $(10 \%$ concentrated $\mathrm{HCl})$. The isolated polymer was collected and dried overnight before GPC and NMR analysis. Details on the amount of the reagents, polymerization time, and polymer characterization are provided in Table 1 (main text).

LCCTP of 1,5-hexadiene. In a $25 \mathrm{~mL}$ round bottom, to a $5 \mathrm{~mL}$ toluene solution of 38.0 $\mathrm{mg}(0.048 \mathrm{mmol})$ of $\left[\mathrm{PhNMe}_{2} \mathrm{H}\right]\left[\mathrm{B}\left(\mathrm{C}_{6} \mathrm{~F}_{5}\right)_{4}\right]$ chilled to $-5{ }^{\circ} \mathrm{C}$ was added $24.6 \mathrm{mg}(0.044$ $\mathrm{mmol})$ of $\left(\boldsymbol{S}_{\mathrm{c}}, \boldsymbol{S}_{H \mathrm{H}}\right)-1$ in $1 \mathrm{~mL}$ of chlorobenzene prechilled to $-5^{\circ} \mathrm{C}$. The solution was stirred and allowed to equilibrate for 20 minutes, turning a pale-yellow color. $55.0 \mathrm{mg}(0.450$ $\mathrm{mmol})$ of $\mathrm{ZnEt}_{2}$ was added to the solution followed by $1.80 \mathrm{~g}(21.9 \mathrm{mmol})$ of 1,5 -hexadiene added all at once. The reaction mixture was stirred for 24 hours at $-5^{\circ} \mathrm{C}$ before quenching with $2.0 \mathrm{~mL}$ of acidic methanol $(10 \%$ concentrated $\mathrm{HCl})$. Solvent was removed in vacuo and residue was collected in hexanes and passed through a silica plug. Solvent was removed in vacuo and the isolated polymer was dried overnight before GPC and NMR analysis. Details on the amount of the reagents, polymerization time, and polymer characterization are provided in Table 1 (main text).

LCCTP of 1,5-hexadiene with iodo termination. In a $25 \mathrm{~mL}$ round bottom, to a $5 \mathrm{~mL}$ toluene solution of $38.0 \mathrm{mg}(0.048 \mathrm{mmol})$ of $\left[\mathrm{PhNMe}{ }_{2} \mathrm{H}\right]\left[\mathrm{B}\left(\mathrm{C}_{6} \mathrm{~F}_{5}\right)_{4}\right]$ chilled to $-5{ }^{\circ} \mathrm{C}$ was added $24.6 \mathrm{mg}(0.044 \mathrm{mmol})$ of $\left(\boldsymbol{S}_{\mathbf{c}}, \boldsymbol{S}_{H f}\right)-1$ in $1 \mathrm{~mL}$ of chlorobenzene prechilled to $-5^{\circ} \mathrm{C}$. The solution was stirred and allowed to equilibrate for 20 minutes, turning a pale-yellow color. $55.0 \mathrm{mg}(0.450 \mathrm{mmol})$ of $\mathrm{ZnEt}_{2}$ was added to the solution followed by $1.80 \mathrm{~g}(21.9$ $\mathrm{mmol}$ ) of 1,5-hexadiene added all at once. The reaction mixture was stirred 24 hours at $5^{\circ} \mathrm{C}$ before quenching with $0.275 \mathrm{~g}(1.08 \mathrm{mmol})$ of $\mathrm{I}_{2}$. Solvent was removed in vacuo and residue was collected in hexane and passed through a silica plug. Solvent was removed in vacuo and the isolated polymer was dried overnight before GPC and NMR analysis. Details on the amount of the reagents, polymerization time, and polymer characterization are provided in Table 1 (main text). 


\section{Supporting NMR Spectra}

A
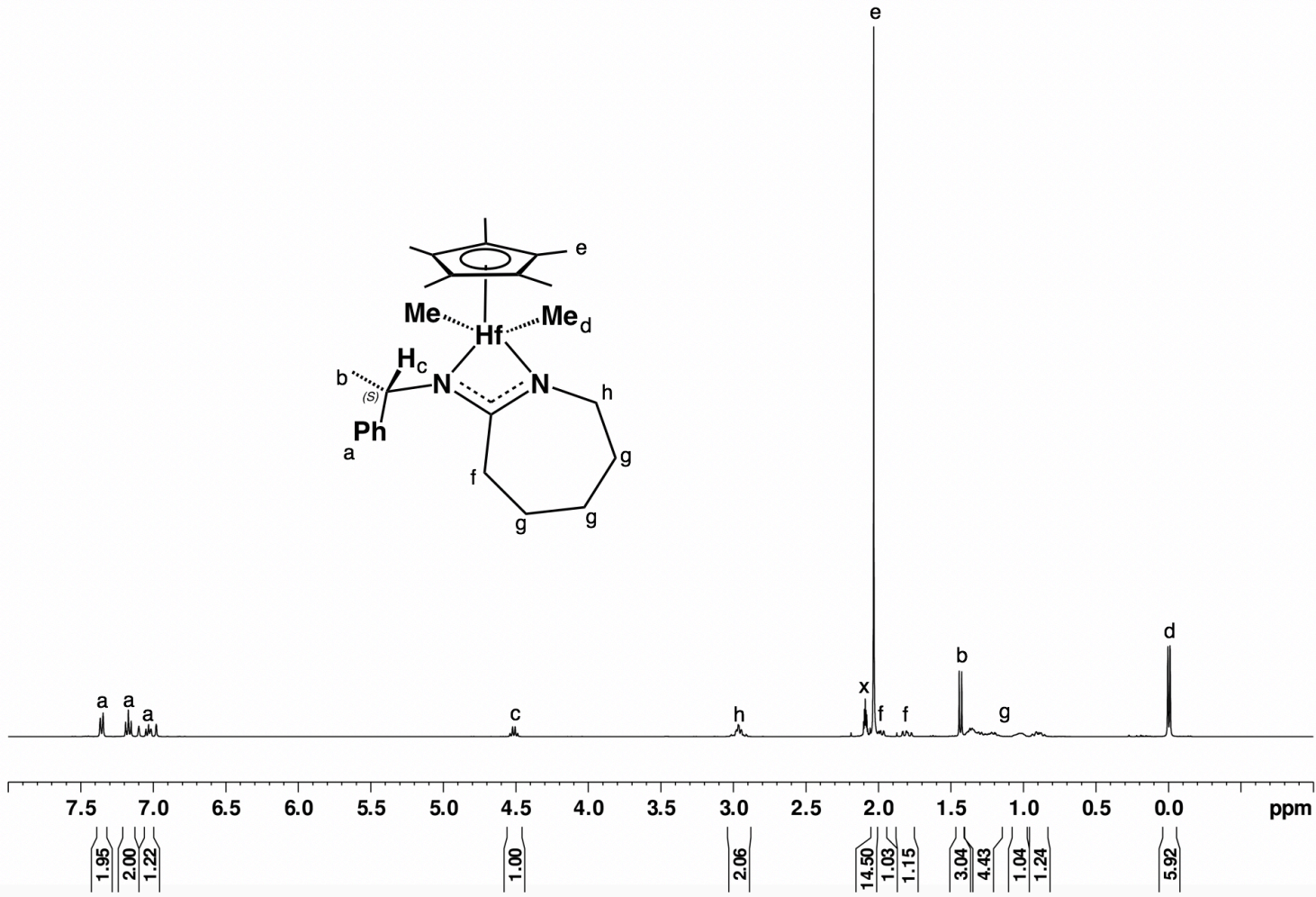

B

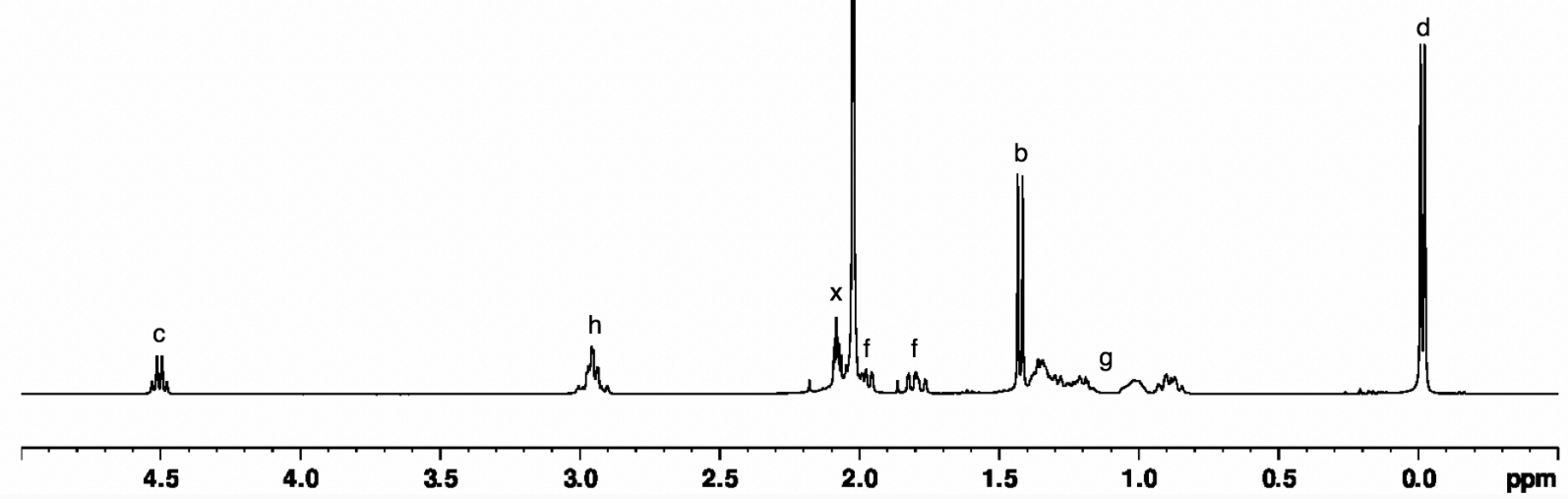

Figure S1. (A) ${ }^{1} \mathrm{H}$ NMR $\left(400 \mathrm{MHz}\right.$, Tol- $\left.d_{8}, 25^{\circ} \mathrm{C}\right)$ spectrum of $\left(\boldsymbol{S}_{\mathrm{c}}, \boldsymbol{S}_{H f}\right)-1$. (B) Expanded ${ }^{1} \mathrm{H}$ NMR of $\left(\boldsymbol{S}_{\mathrm{c}}\right.$, $\left.S_{H f}\right)-1 ; X$ denotes toluene- $d_{8}$ solvent. 
A
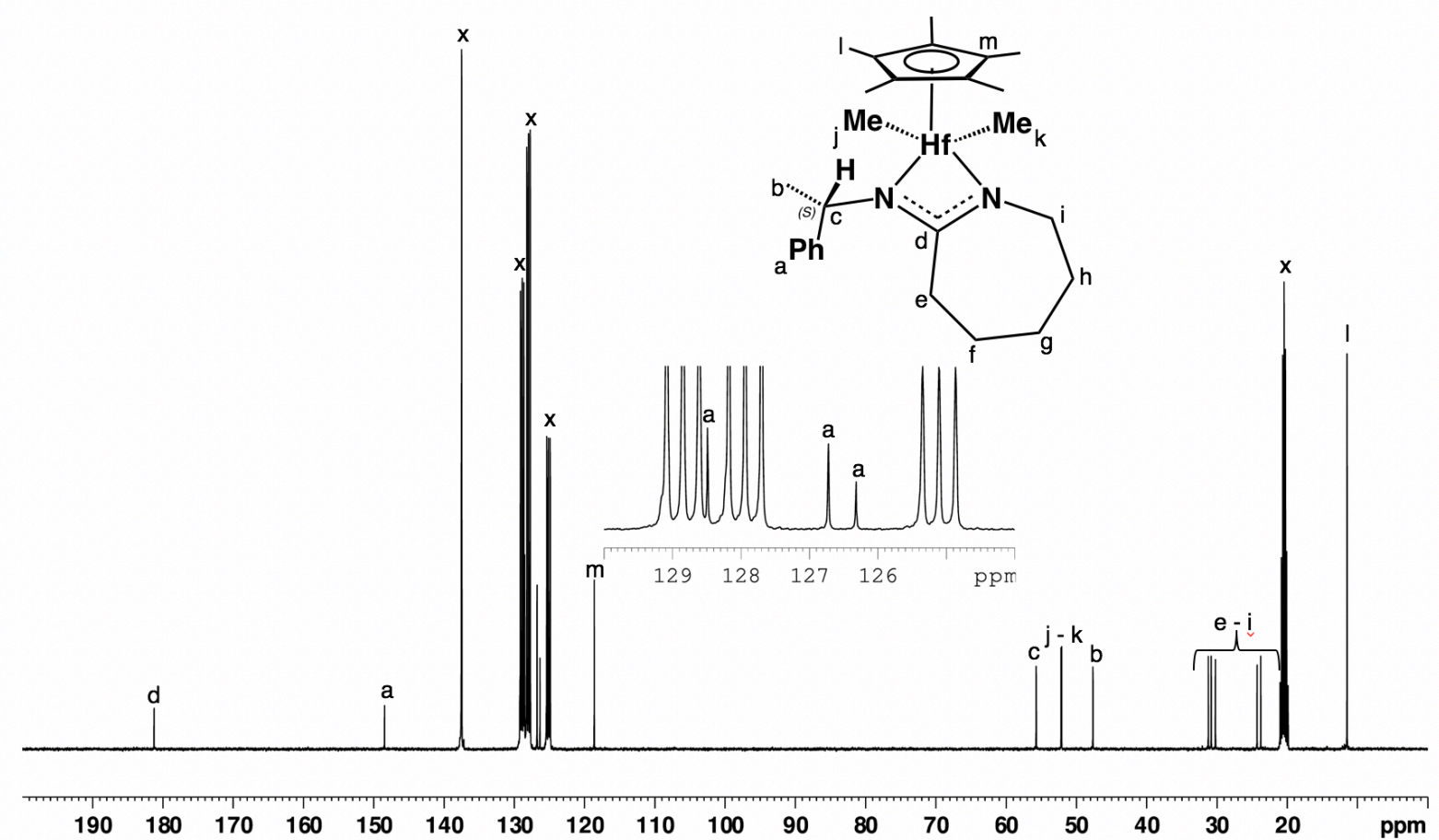

B

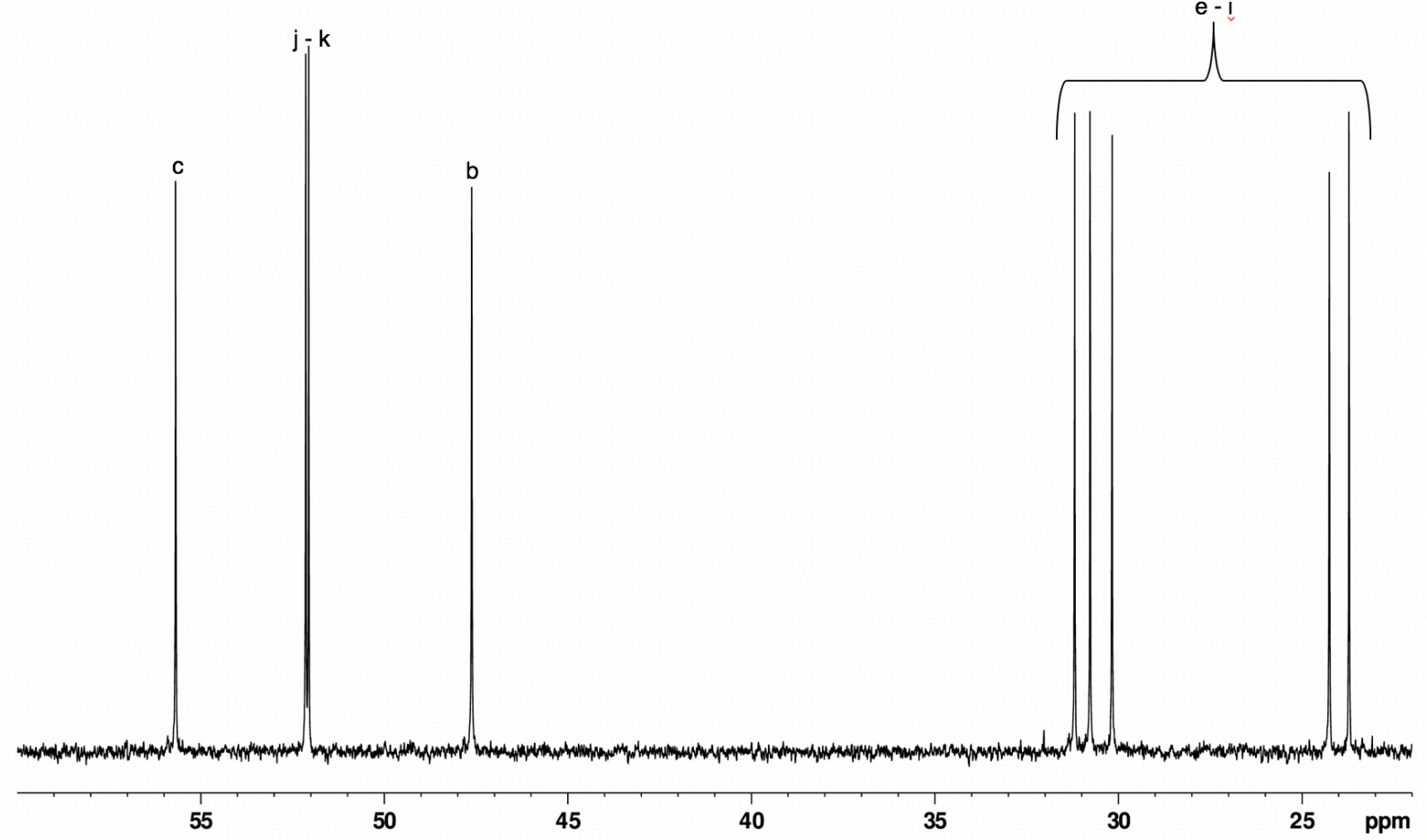

Figure S2. (A) ${ }^{13} \mathrm{C}$ NMR $\left(100 \mathrm{MHz}\right.$, Tol- $\left.d_{8}, 25{ }^{\circ} \mathrm{C}\right)$ spectrum of $\left(\boldsymbol{S}_{\mathrm{c}}, \boldsymbol{S}_{H f}\right)-1$. (B) Expanded ${ }^{13} \mathrm{C}$ NMR of $\left(\boldsymbol{S}_{\mathrm{c}}\right.$, $\left.S_{H f}\right)-1 ; X$ denotes toluene- $d_{8}$ solvent 
A
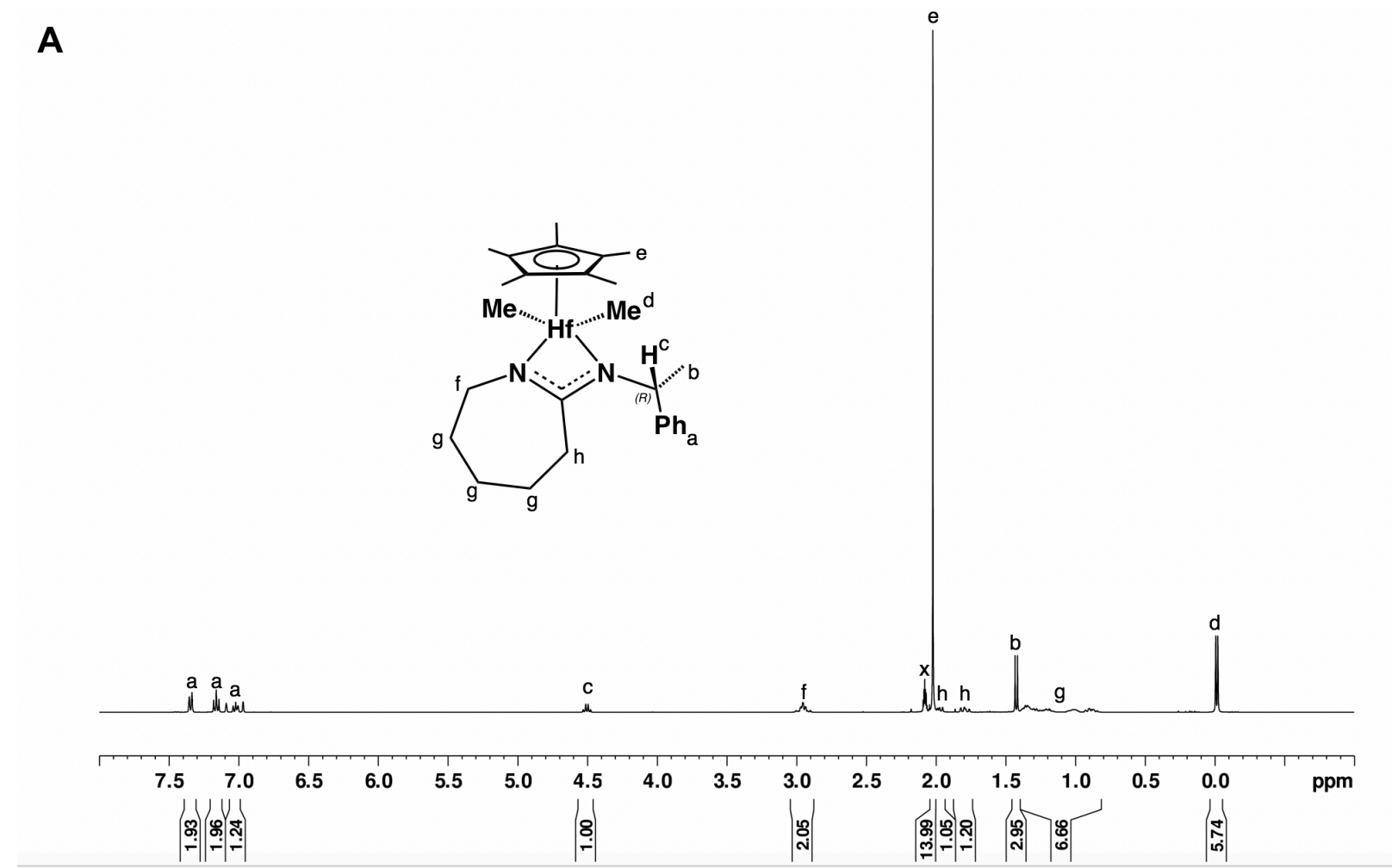

B

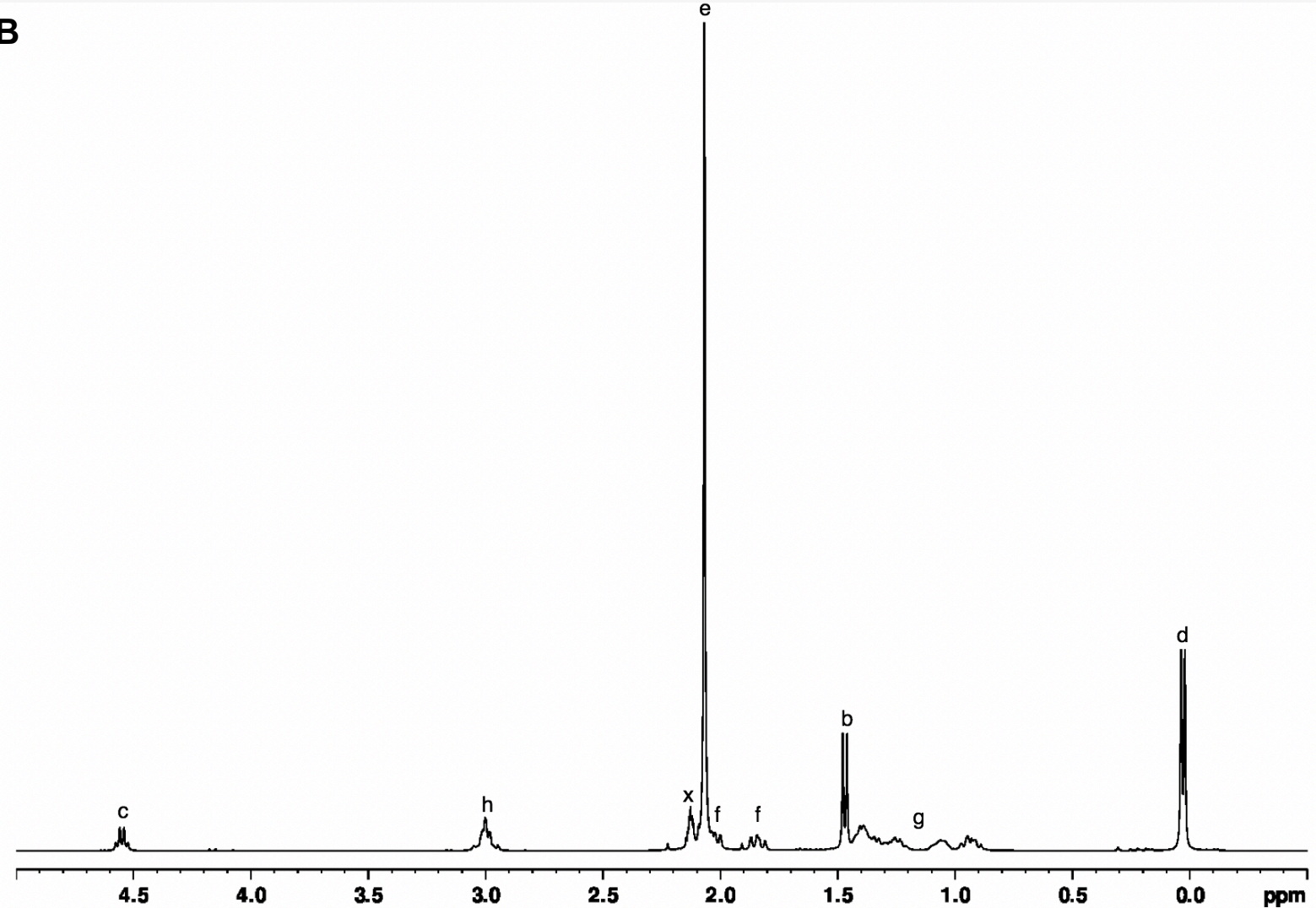

Figure S3. (A) ${ }^{1} \mathrm{H}$ NMR $\left(400 \mathrm{MHz}\right.$, Tol-d $\left.d_{8}, 25^{\circ} \mathrm{C}\right)$ spectrum of $\left(\boldsymbol{R}_{\mathbf{c}}, \boldsymbol{R}_{\boldsymbol{H}} \mathrm{f}\right)$-1. (B) Expanded ${ }^{1} \mathrm{H}$ NMR spectrum of $\left(\boldsymbol{R}_{\mathrm{c}}, \boldsymbol{R}_{H f}\right)-\mathbf{1} ; \mathbf{X}$ denotes toluene- $d_{8}$ solvent 
A

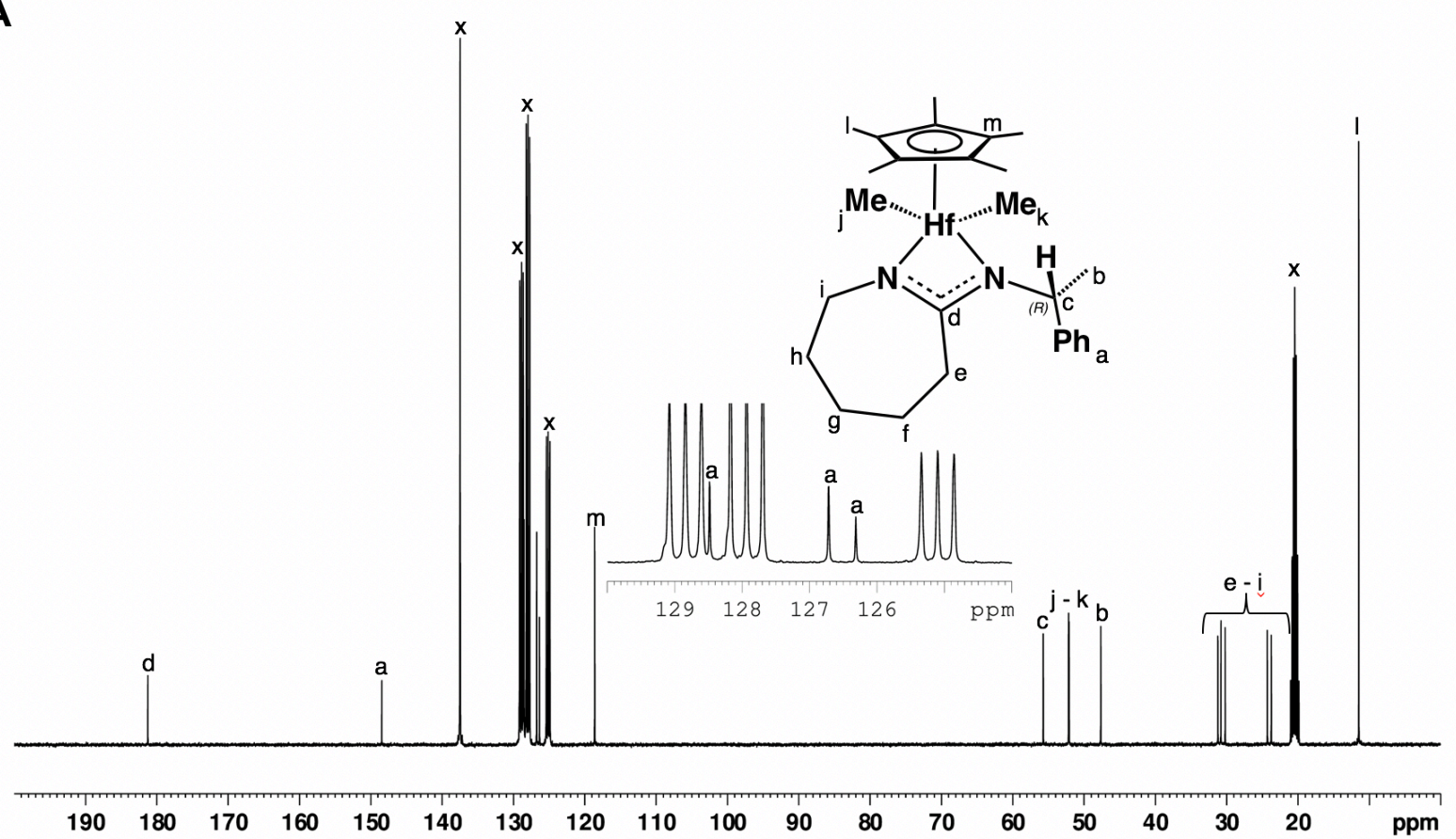

B

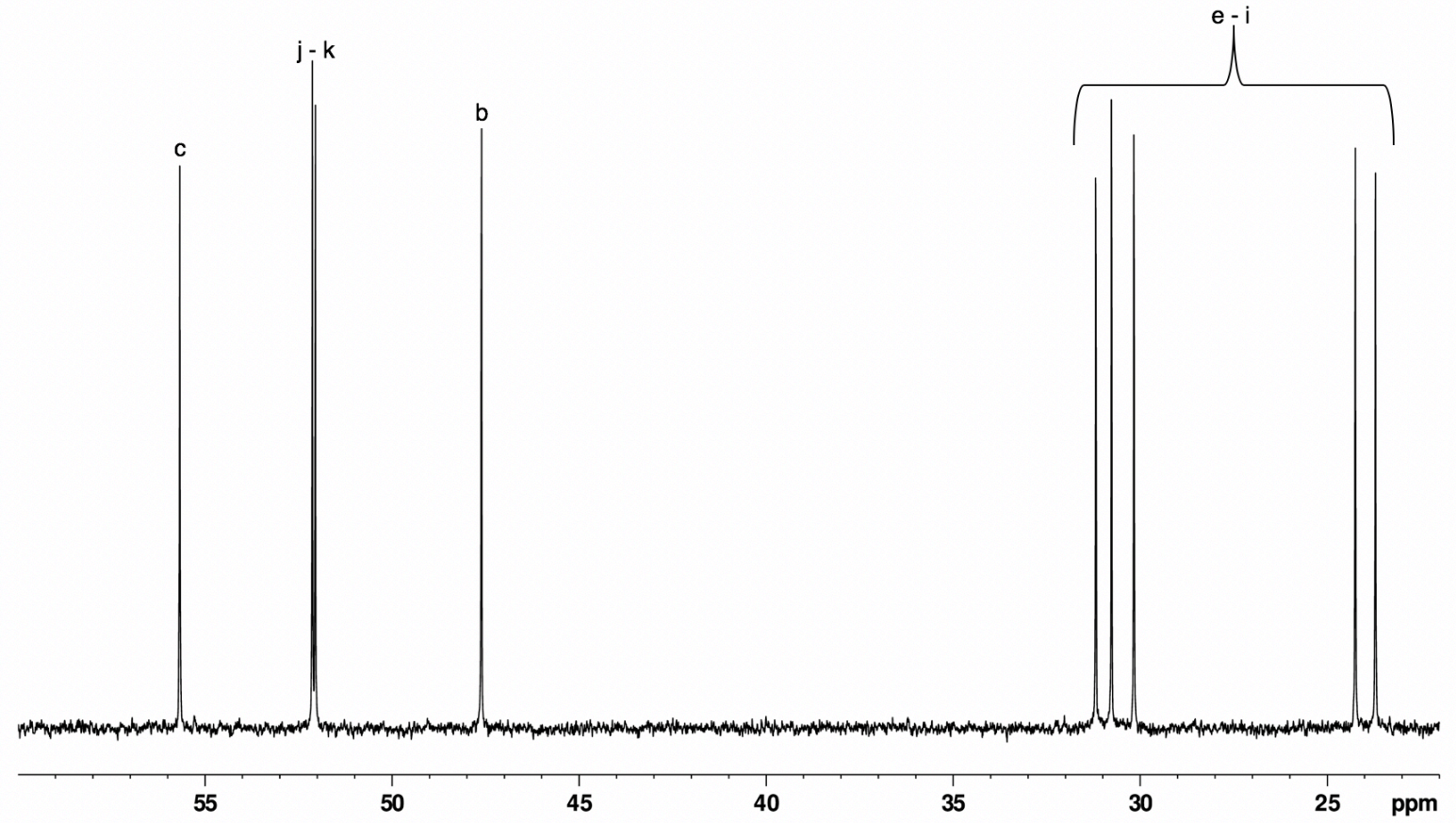

Figure S4. (A) ${ }^{13} \mathrm{C}$ NMR $\left(100 \mathrm{MHz}\right.$, Tol- $\left.d_{8}, 25{ }^{\circ} \mathrm{C}\right)$ spectrum of $\left(\boldsymbol{R}_{\mathrm{c}}, \boldsymbol{R}_{H f}\right)-1$. (B) Expanded ${ }^{13} \mathrm{C}$ NMR of $\left(\boldsymbol{R}_{\mathrm{c}}\right.$, $\left.\boldsymbol{R}_{H f}\right)-\mathbf{1} ; \mathbf{X}$ denotes toluene- $d_{8}$ solvent 
A
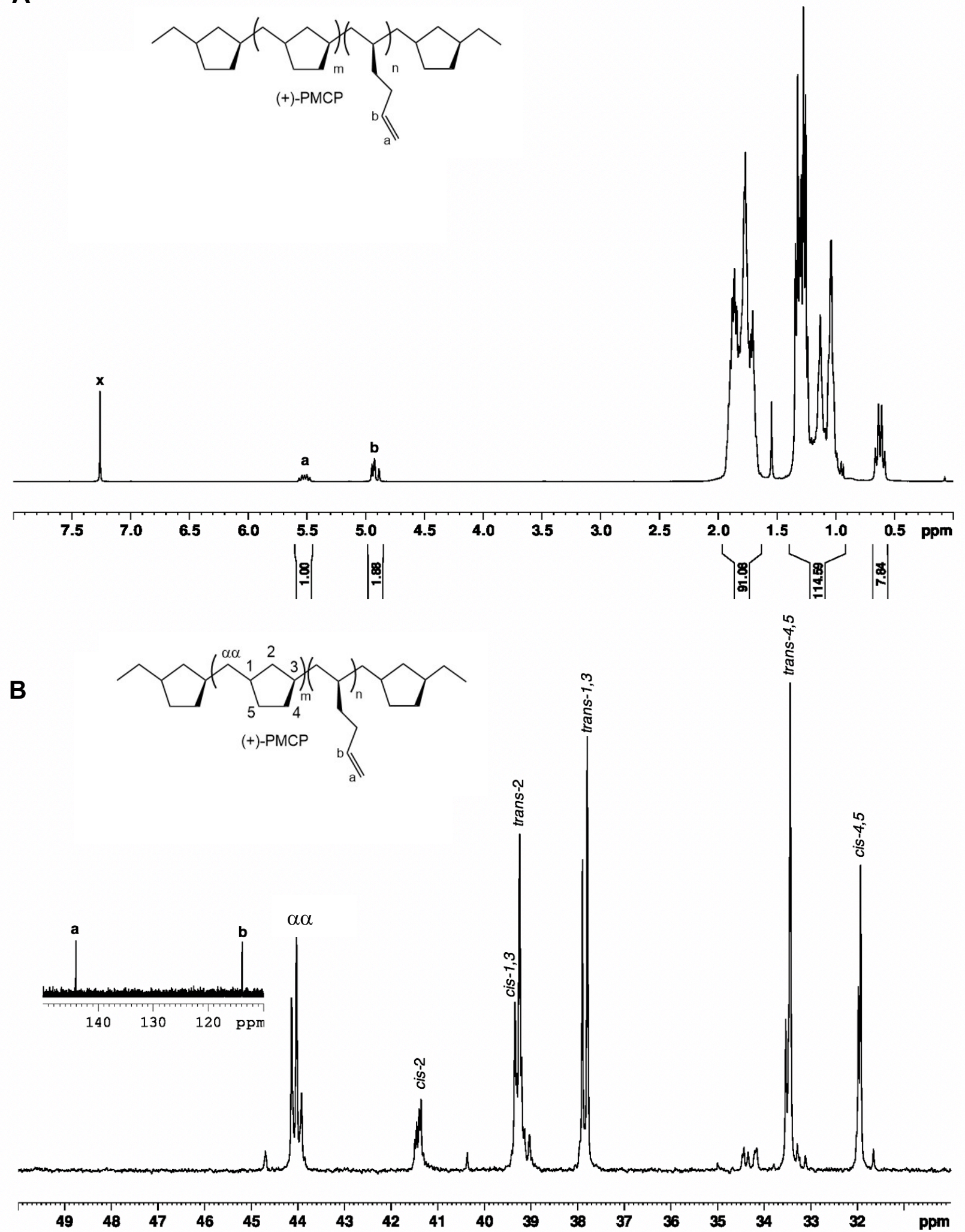

Figure S5. (A) ${ }^{1} \mathrm{H}$ NMR $\left(400 \mathrm{MHz}, \mathrm{CDCl}_{3}, 25^{\circ} \mathrm{C}\right)$ spectrum of PMCP from run 1. (B) Partial ${ }^{13} \mathrm{C}$ NMR $(100$ $\mathrm{MHz}, \mathrm{CDCl}_{3}, 25^{\circ} \mathrm{C}$ ) spectrum of PMCP from run $\mathbf{1} ; \mathbf{X}$ denotes $\mathrm{CDCl}_{3}$ solvent. 
A
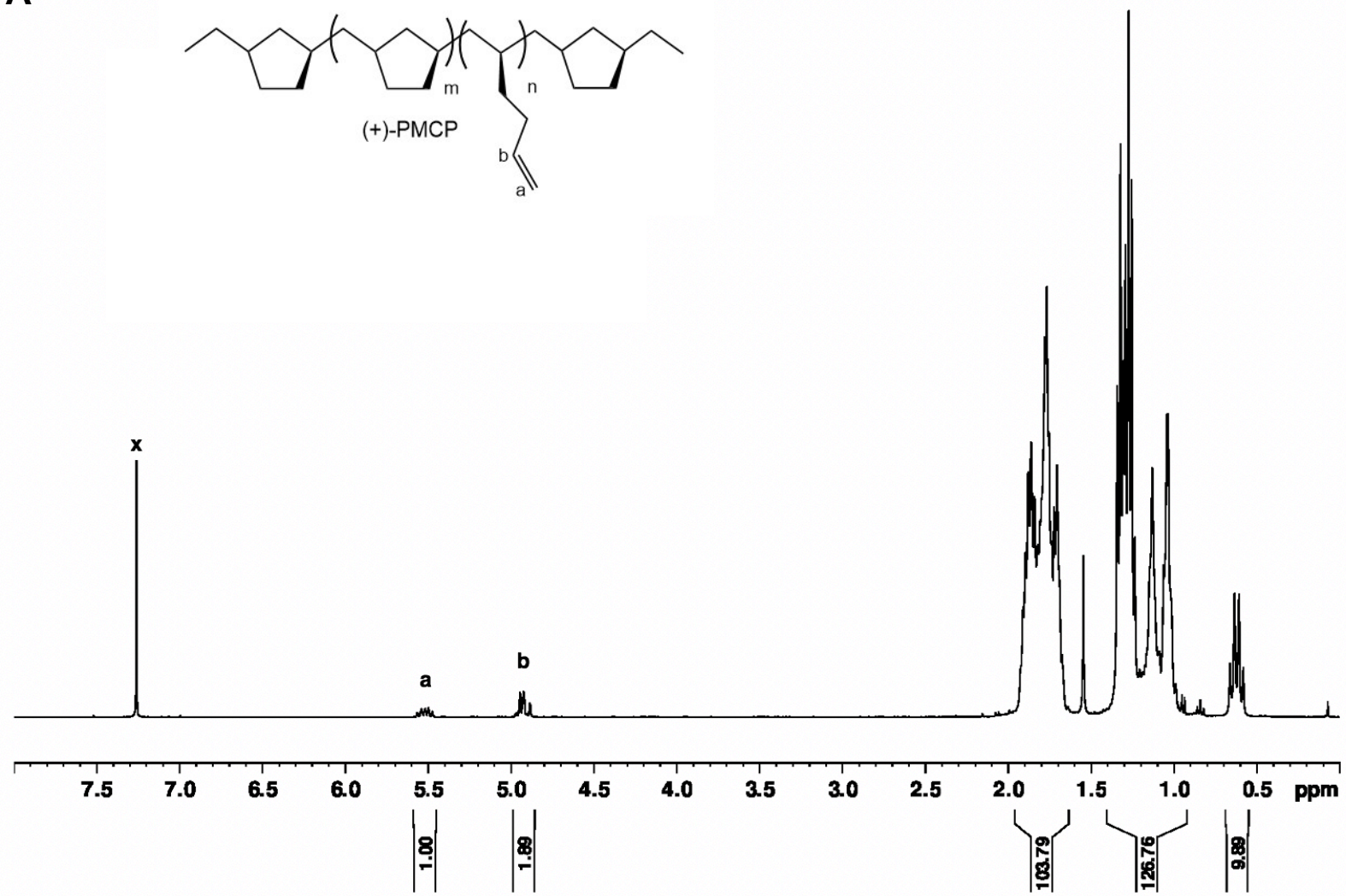

B

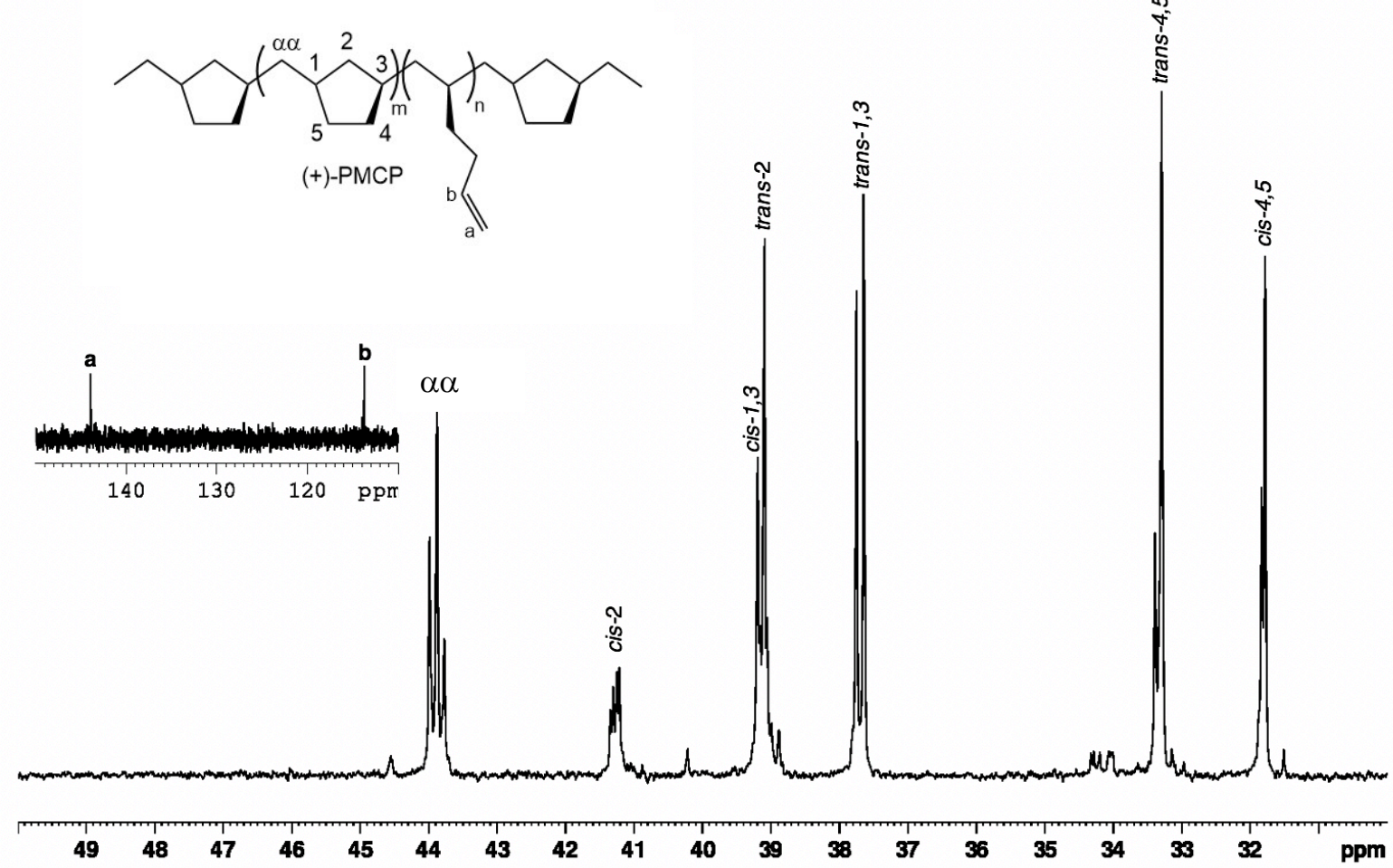

Figure S6. (A) ${ }^{1} \mathrm{H}$ NMR $\left(400 \mathrm{MHz}, \mathrm{CDCl}_{3}, 25^{\circ} \mathrm{C}\right)$ spectrum of $\mathrm{PMCP}$ from run 2. (B) Partial ${ }^{13} \mathrm{C}$ NMR $\left(100 \mathrm{MHz}, \mathrm{CDCl}_{3}, 25^{\circ} \mathrm{C}\right.$ ) spectrum of PMCP from run 2; $\mathbf{X}$ denotes $\mathrm{CDCl}_{3}$ solvent 
A

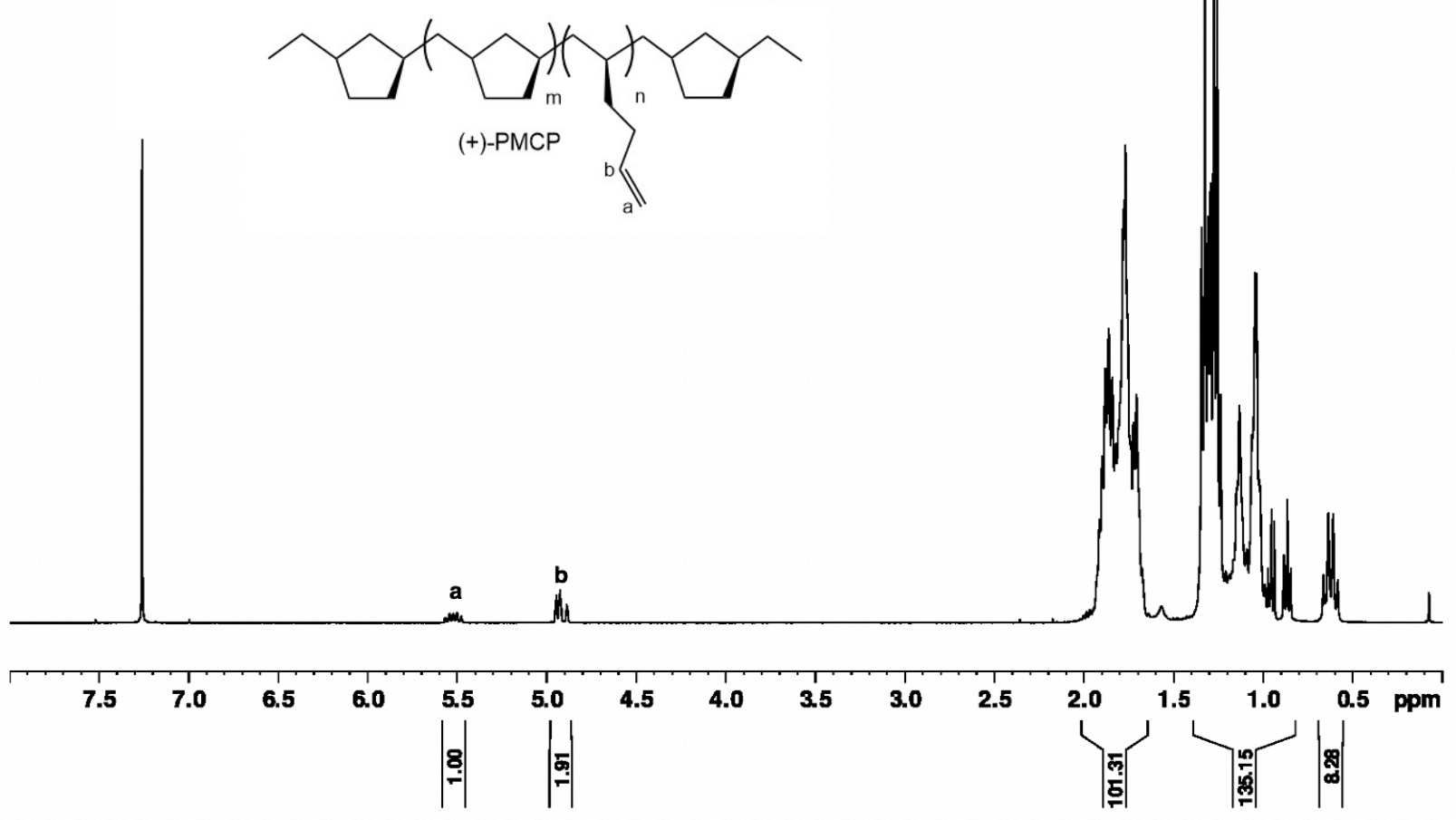

B

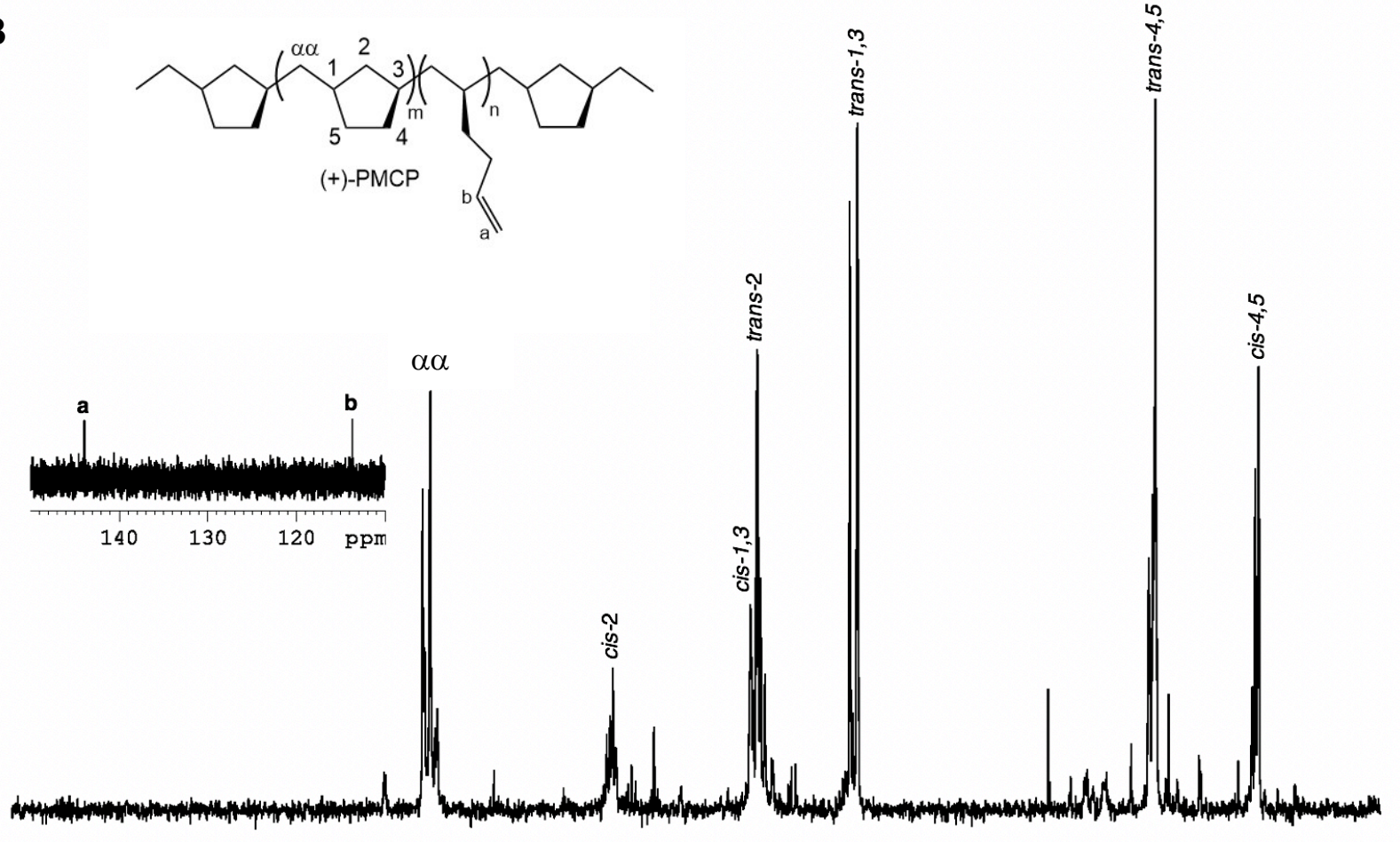

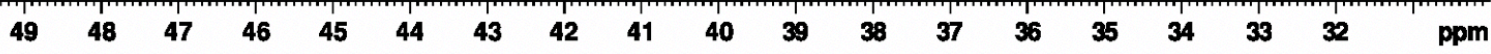

Figure S7. (A) ${ }^{1} \mathrm{H}$ NMR $\left(400 \mathrm{MHz}, \mathrm{CDCl}_{3}, 25^{\circ} \mathrm{C}\right)$ spectrum of PMCP from run 3. (B) Partial ${ }^{13} \mathrm{C} \mathrm{NMR}$ $\left(100 \mathrm{MHz}, \mathrm{CDCl}_{3}, 25^{\circ} \mathrm{C}\right.$ ) spectrum of $\mathrm{PMCP}$ from run 3; $\mathbf{X}$ denotes $\mathrm{CDCl}_{3}$ solvent. 
A<smiles>C=CCC[C@@H](CC1CCC(CC2CCC(CC)C2)C1)CC(C)C1CCC(CC)C1</smiles>

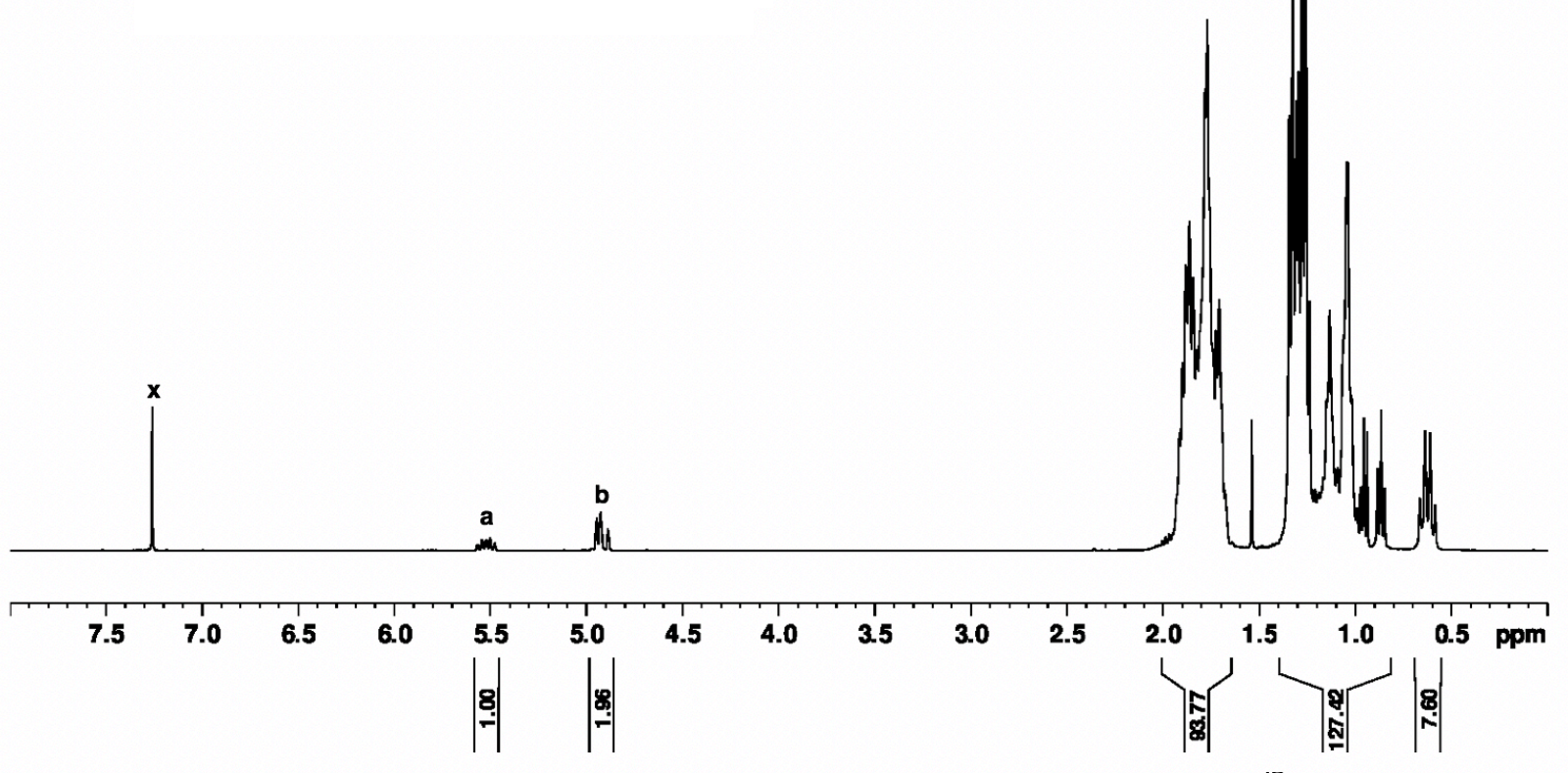

B

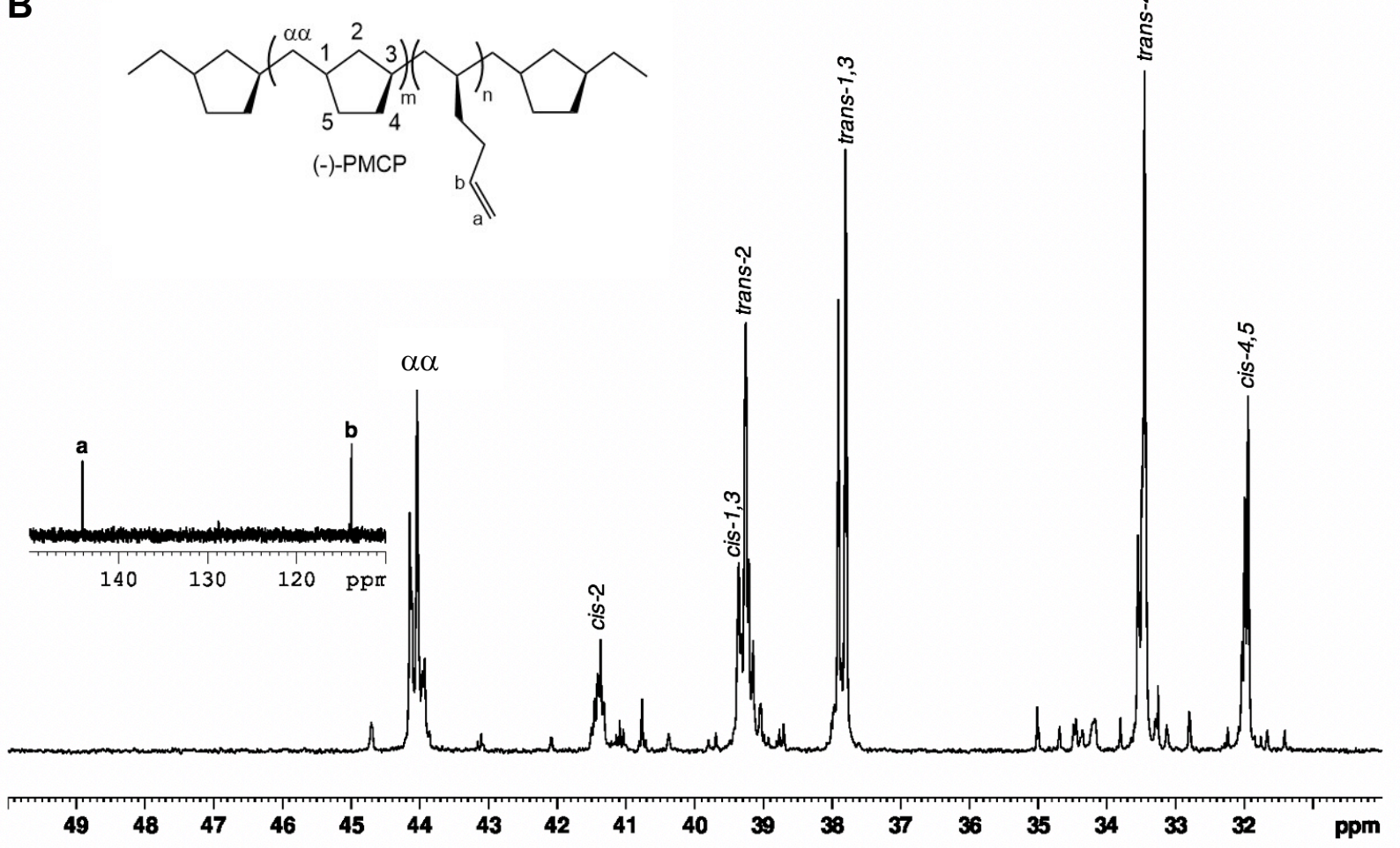

Figure S8. (A) ${ }^{1} \mathrm{H}$ NMR $\left(400 \mathrm{MHz}, \mathrm{CDCl}_{3}, 25{ }^{\circ} \mathrm{C}\right)$ spectrum of PMCP from run 4. (B) Partial ${ }^{13} \mathrm{C}$ NMR $\left(100 \mathrm{MHz}, \mathrm{CDCl}_{3}, 25^{\circ} \mathrm{C}\right.$ ) spectrum of PMCP from run $4 ; \mathbf{X}$ denotes $\mathrm{CDCl}_{3}$ solvent. 
A<smiles>C=CCCC(CC(C)CC1CCC(CC)C1)CC1CCC(CC)C1</smiles>
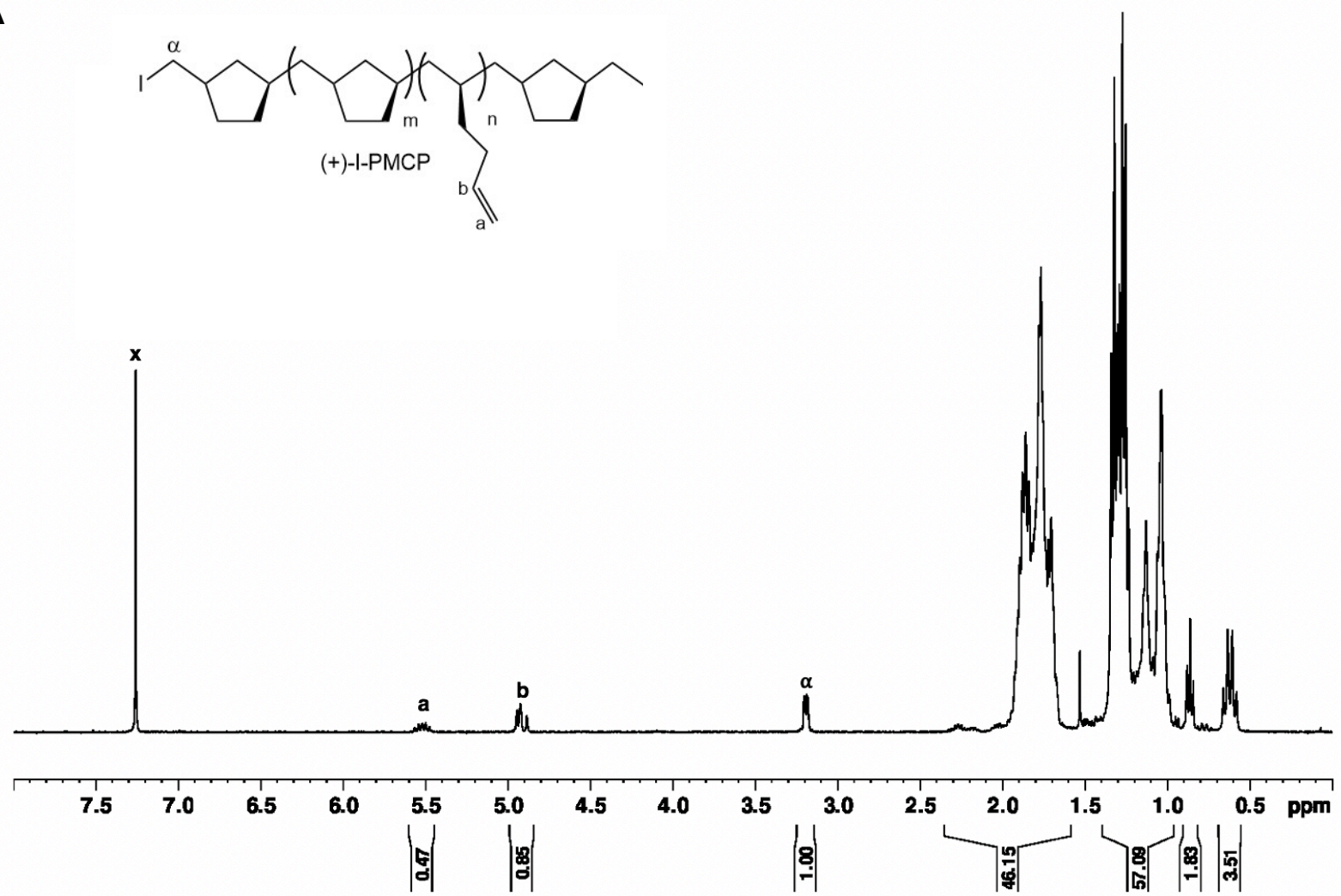

B
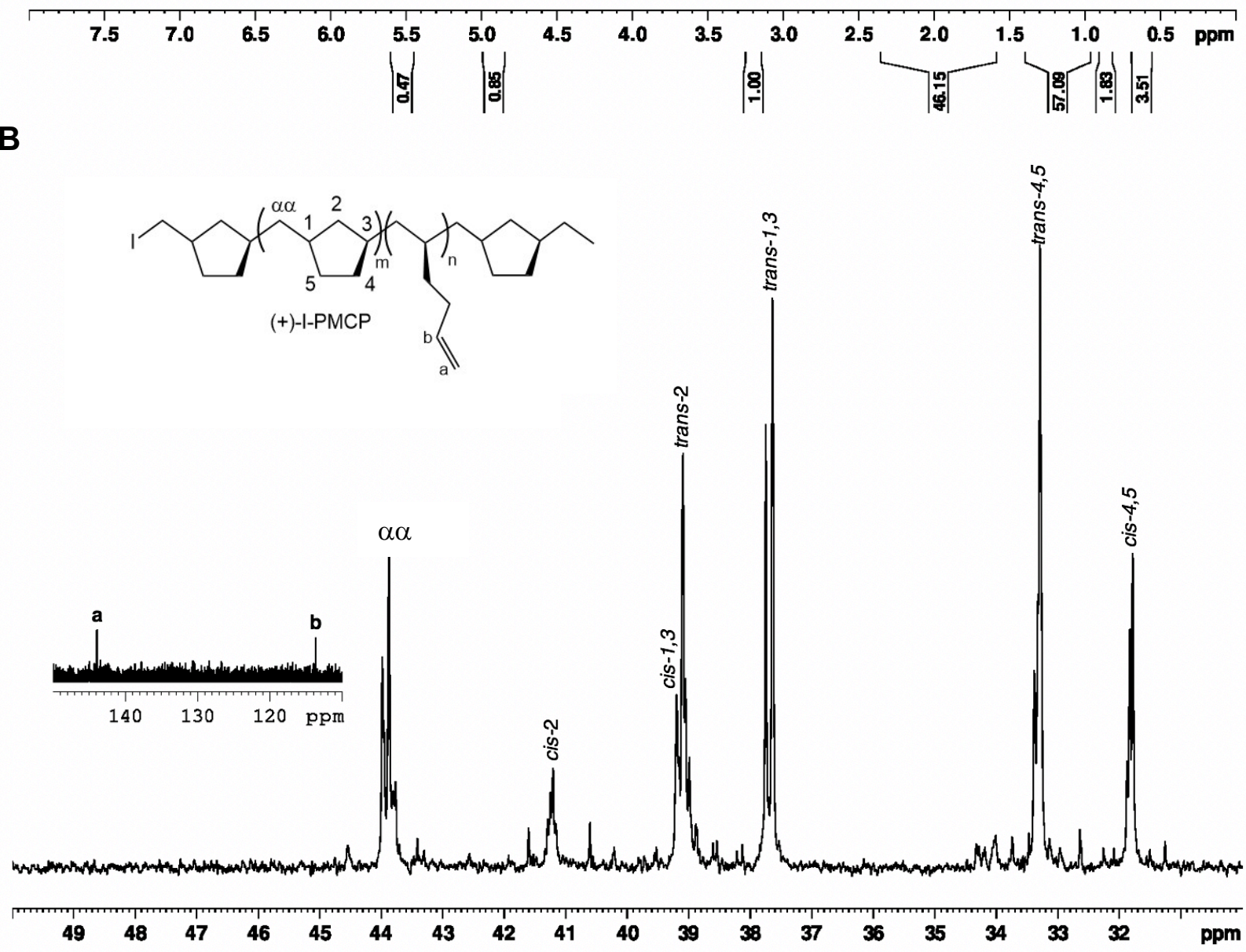

Figure S9. (A) ${ }^{1} \mathrm{H}$ NMR $\left(400 \mathrm{MHz}, \mathrm{CDCl}_{3}, 25^{\circ} \mathrm{C}\right)$ spectrum of I-PMCP from run 5. (B) Partial ${ }^{13} \mathrm{C} \mathrm{NMR}$ $\left(100 \mathrm{MHz}, \mathrm{CDCl}_{3}, 25^{\circ} \mathrm{C}\right.$ ) spectrum of I-PMCP from run $\mathbf{5}$; $\mathbf{X}$ denotes $\mathrm{CDCl}_{3}$ solvent. 
A<smiles>C=CCC[C@@H](CC1CCC(CC2CCC(C[CH]C)C2)C1)CC(C)C1CCC(CC)C1</smiles>
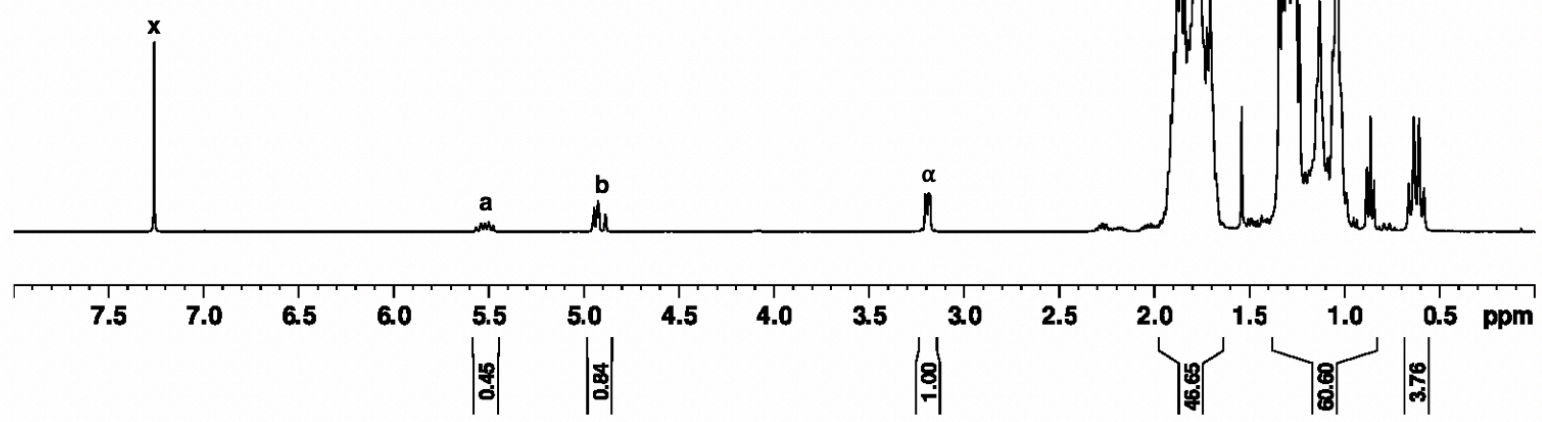

B
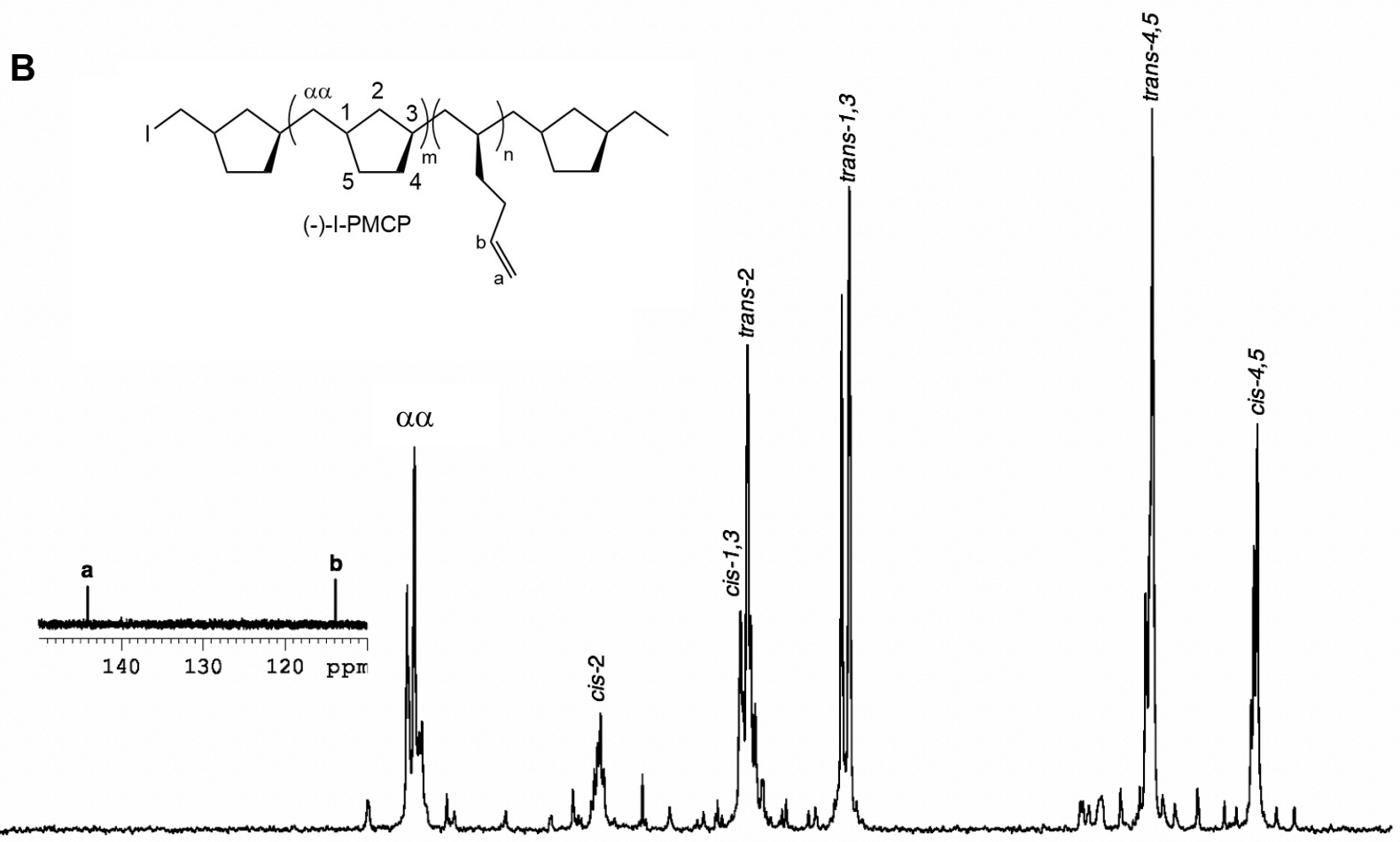

MW Whatura

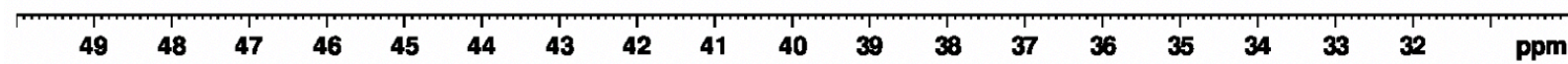

Figure S10. (A) ${ }^{1} \mathrm{H}$ NMR $\left(400 \mathrm{MHz}, \mathrm{CDCl}_{3}, 25^{\circ} \mathrm{C}\right)$ spectrum of I-PMCP from run 6. (B) Partial ${ }^{13} \mathrm{C}$ NMR $\left(100 \mathrm{MHz}, \mathrm{CDCl}_{3}, 25^{\circ} \mathrm{C}\right.$ ) spectrum of I-PMCP from run $\mathbf{6} ; \mathbf{X}$ denotes $\mathrm{CDCl}_{3}$ solvent. 
A
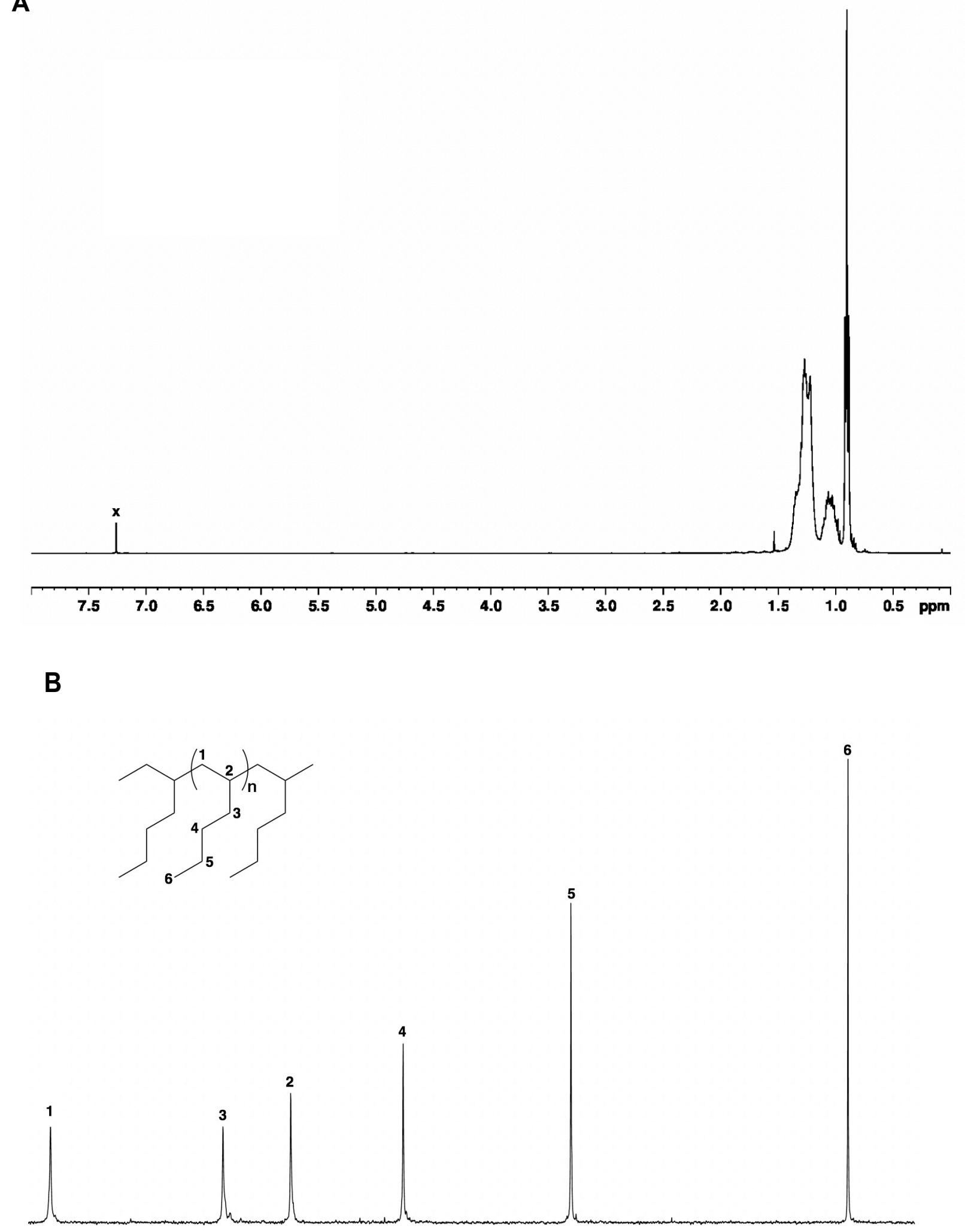

Figure S11. (A) ${ }^{1} \mathrm{H}$ NMR $\left(400 \mathrm{MHz}, \mathrm{CDCl}_{3}, 25^{\circ} \mathrm{C}\right)$ spectrum of isotactic $\mathrm{PH}$. (B) ${ }^{13} \mathrm{C}$ NMR $(100 \mathrm{MHz}$, $\mathrm{CDCl}_{3}, 25^{\circ} \mathrm{C}$ ) spectrum of isotactic $\mathrm{PH}$; 
A
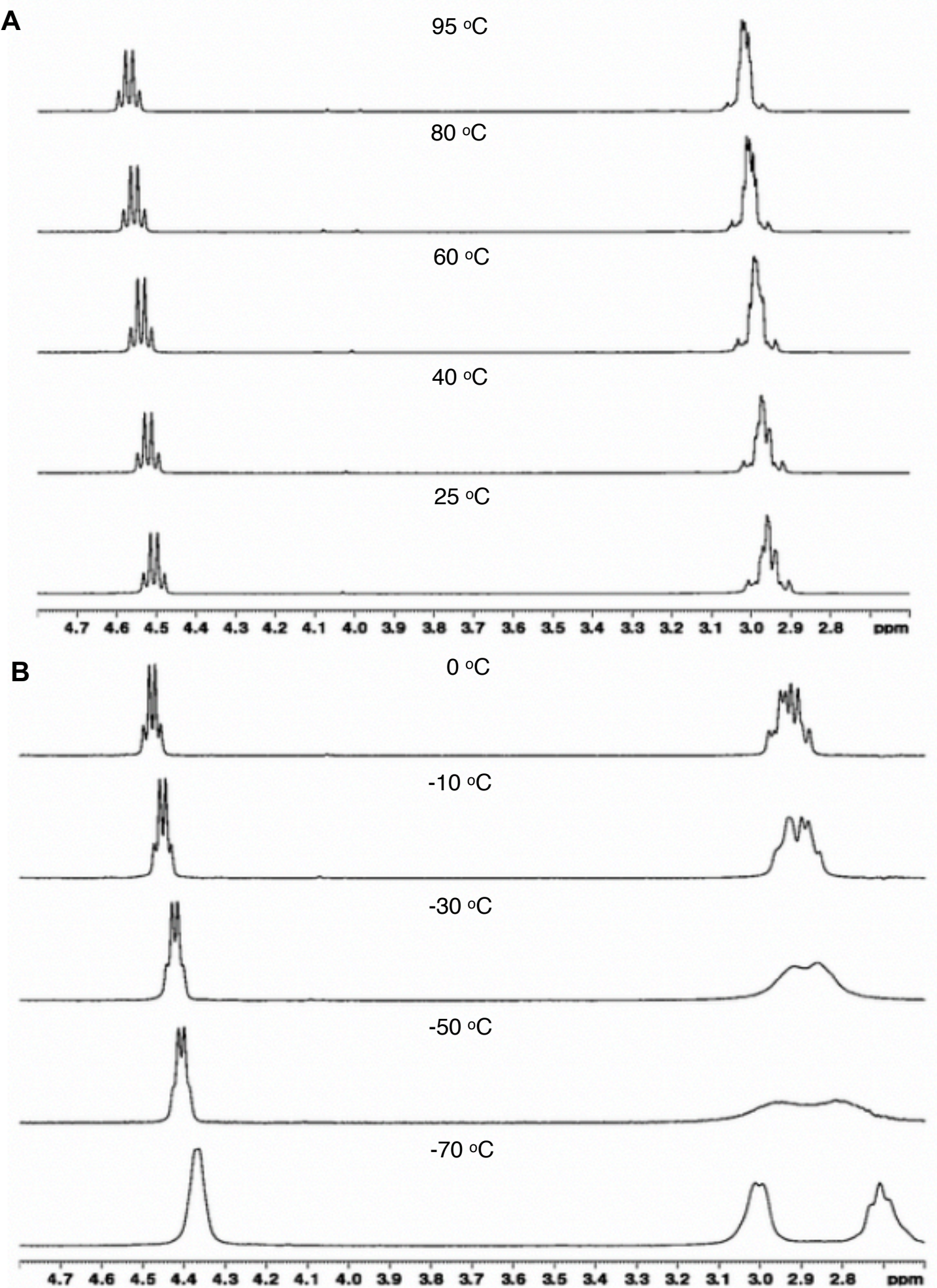
Figure S12. (A) Partial ${ }^{1} \mathrm{H}$ NMR $\left(400 \mathrm{MHz}\right.$, Tol-ds) spectrum of $\left(\boldsymbol{S}_{\mathbf{c}}, \boldsymbol{S}_{H f}\right)-1$ collected from $25^{\circ} \mathrm{C}$ to $95^{\circ} \mathrm{C}$. (B) Partial ${ }^{1} \mathrm{H}$ NMR $\left(500 \mathrm{MHz}\right.$, Tol-ds) spectrum of $\left(\boldsymbol{S}_{\mathbf{c}}, \boldsymbol{S}_{H f}\right)-1$ collected from $-70^{\circ} \mathrm{C}$ to $0{ }^{\circ} \mathrm{C}$.

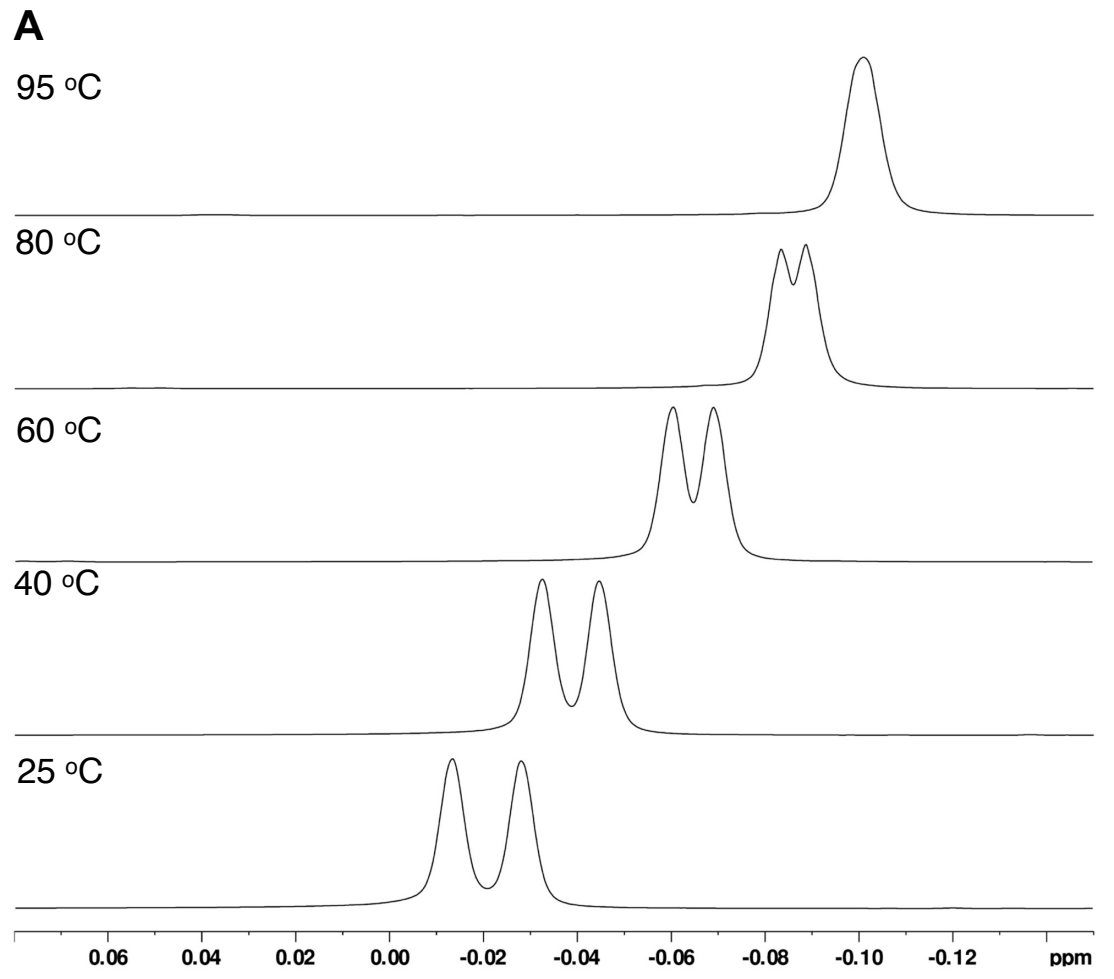

B

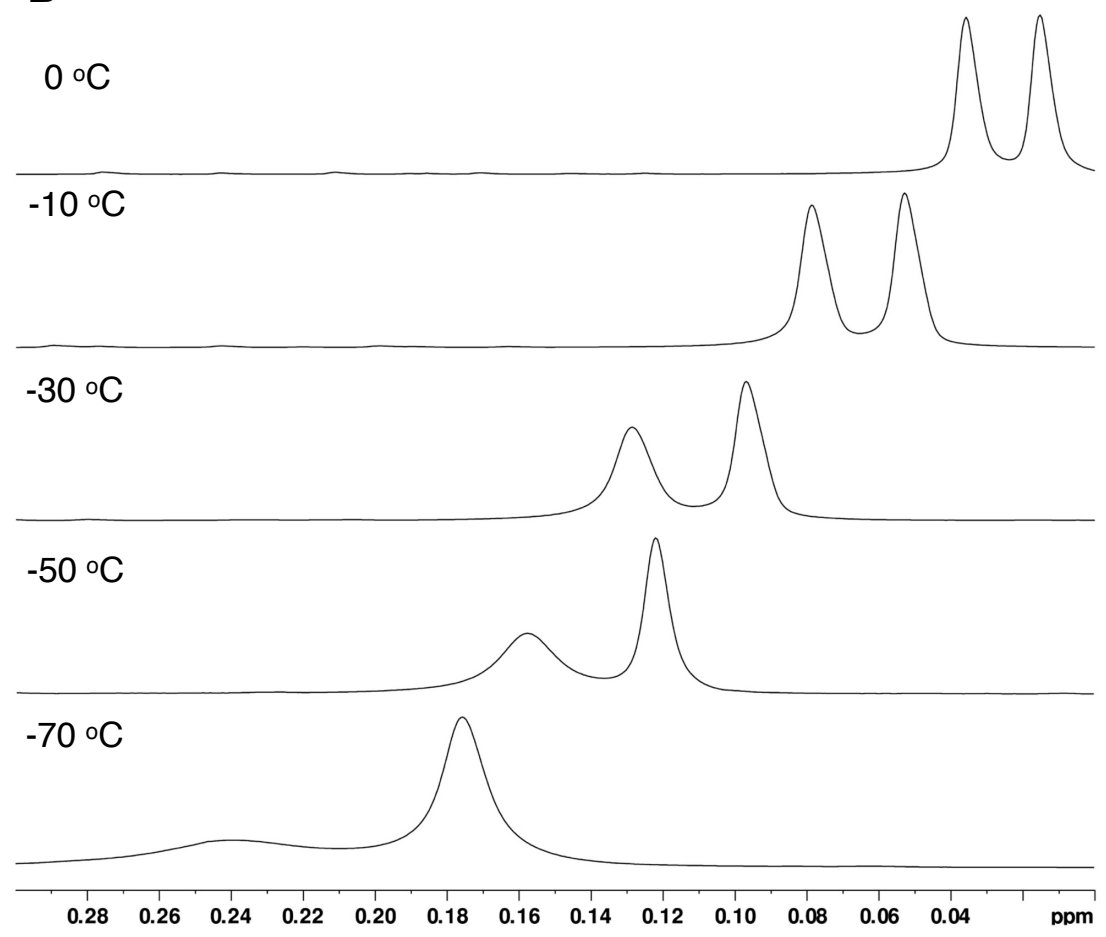

Figure S13. (A) Partial ${ }^{1} \mathrm{H}$ NMR $\left(400 \mathrm{MHz}\right.$, Tol- $\left.d_{8}\right)$ spectrum showing $\mathrm{Hf}-\mathrm{Me}_{2}$ resonances of $\left(\boldsymbol{S}_{\mathrm{c}}, \boldsymbol{S}_{\mathrm{Hf}}\right)-1$ collected from $25^{\circ} \mathrm{C}$ to $95^{\circ} \mathrm{C}$. (B) Partial ${ }^{1} \mathrm{H}$ NMR $\left(500 \mathrm{MHz}\right.$, Tol- $\left.d_{8}\right)$ spectrum showing the $\mathrm{Hf}-\mathrm{Me}_{2}$ resonances of $\left(\boldsymbol{S}_{\mathrm{c}}, \boldsymbol{S}_{H \mathrm{f}}\right)-\mathbf{1}$ collected from $-70^{\circ} \mathrm{C}$ to $0^{\circ} \mathrm{C}$. 


\section{Supporting GPC Traces}

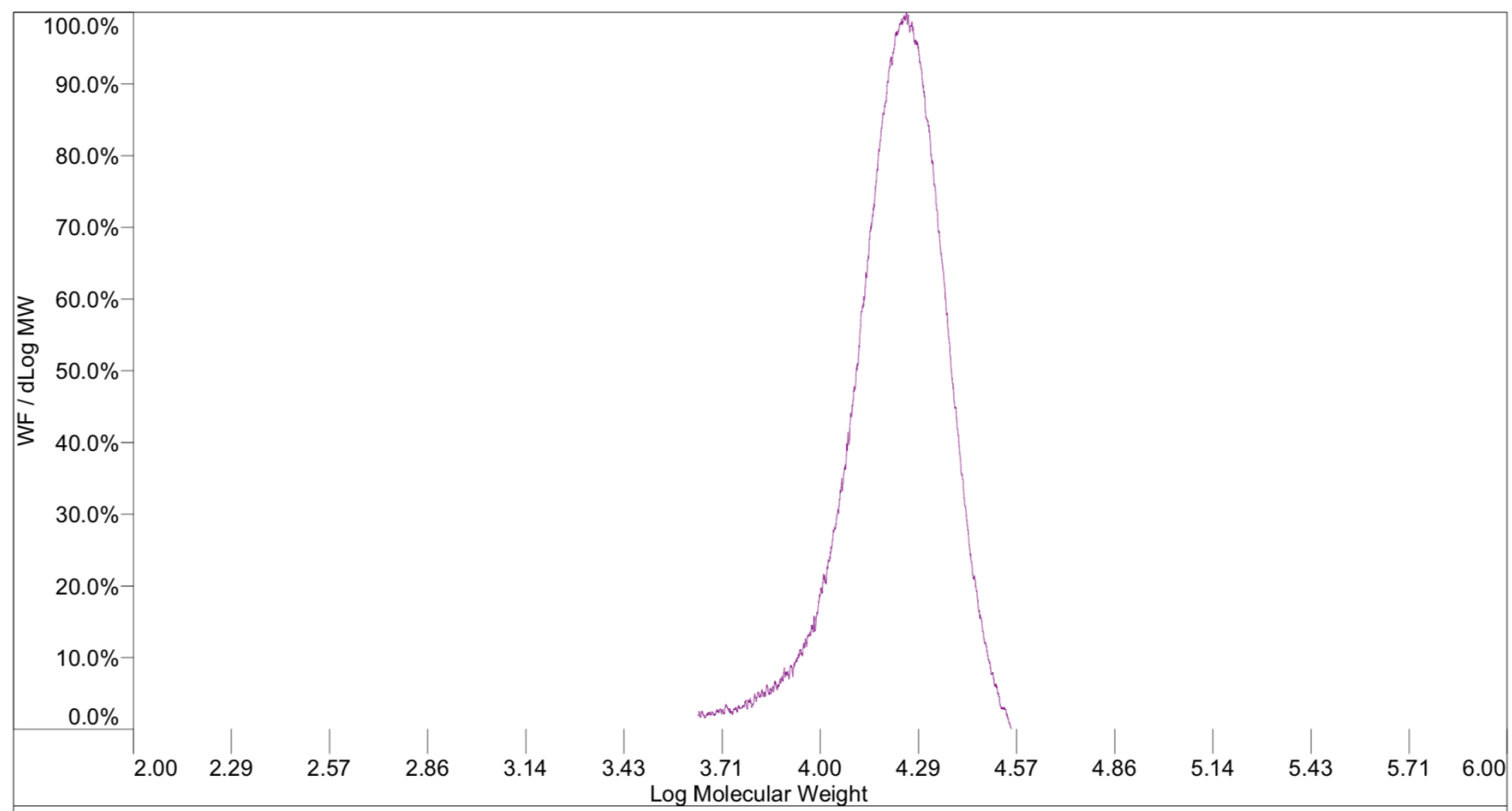

Figure S14. GPC trace of PMCP from run 1.

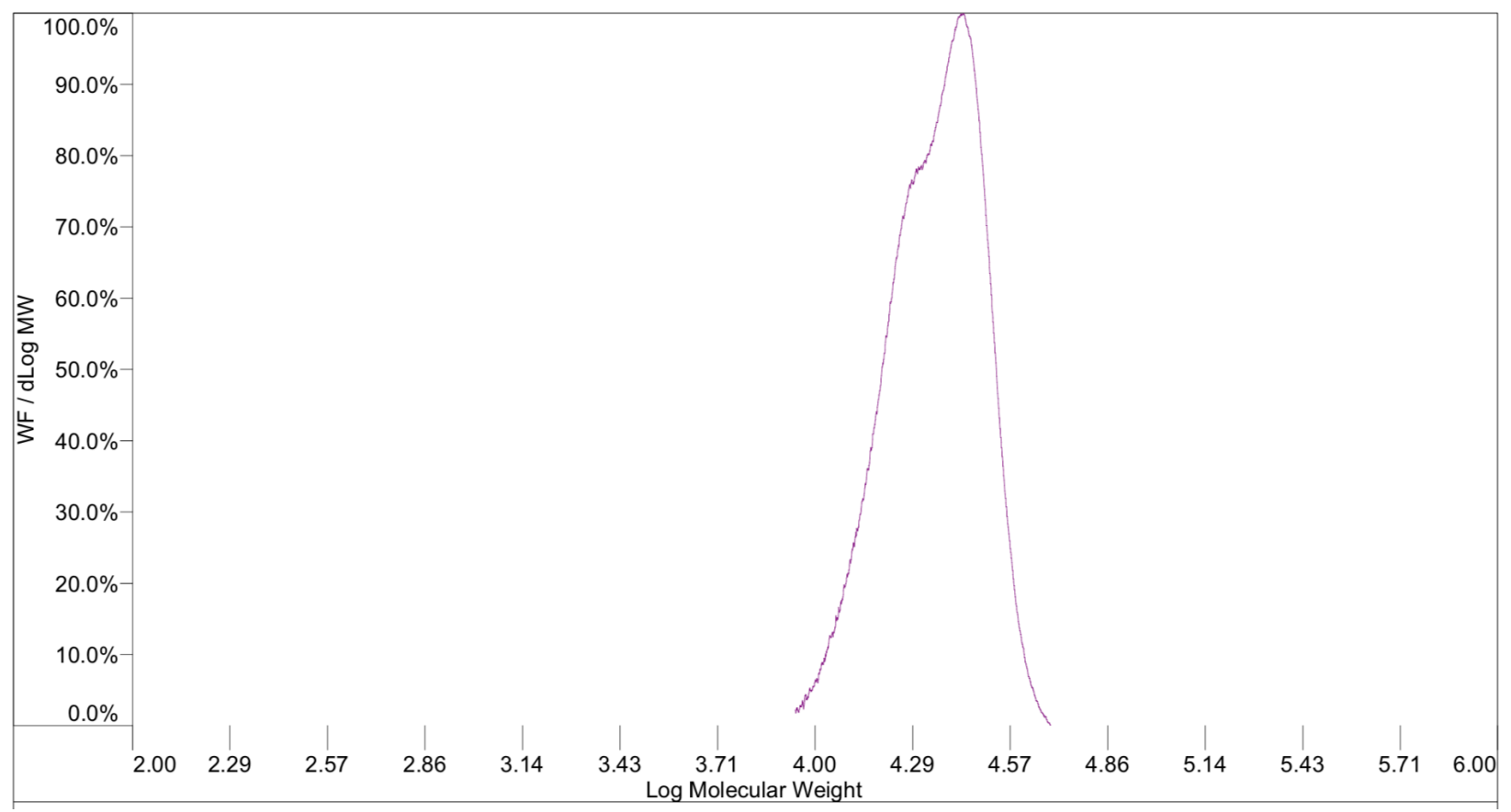

Figure S15. GPC trace of PMCP from run 2. 


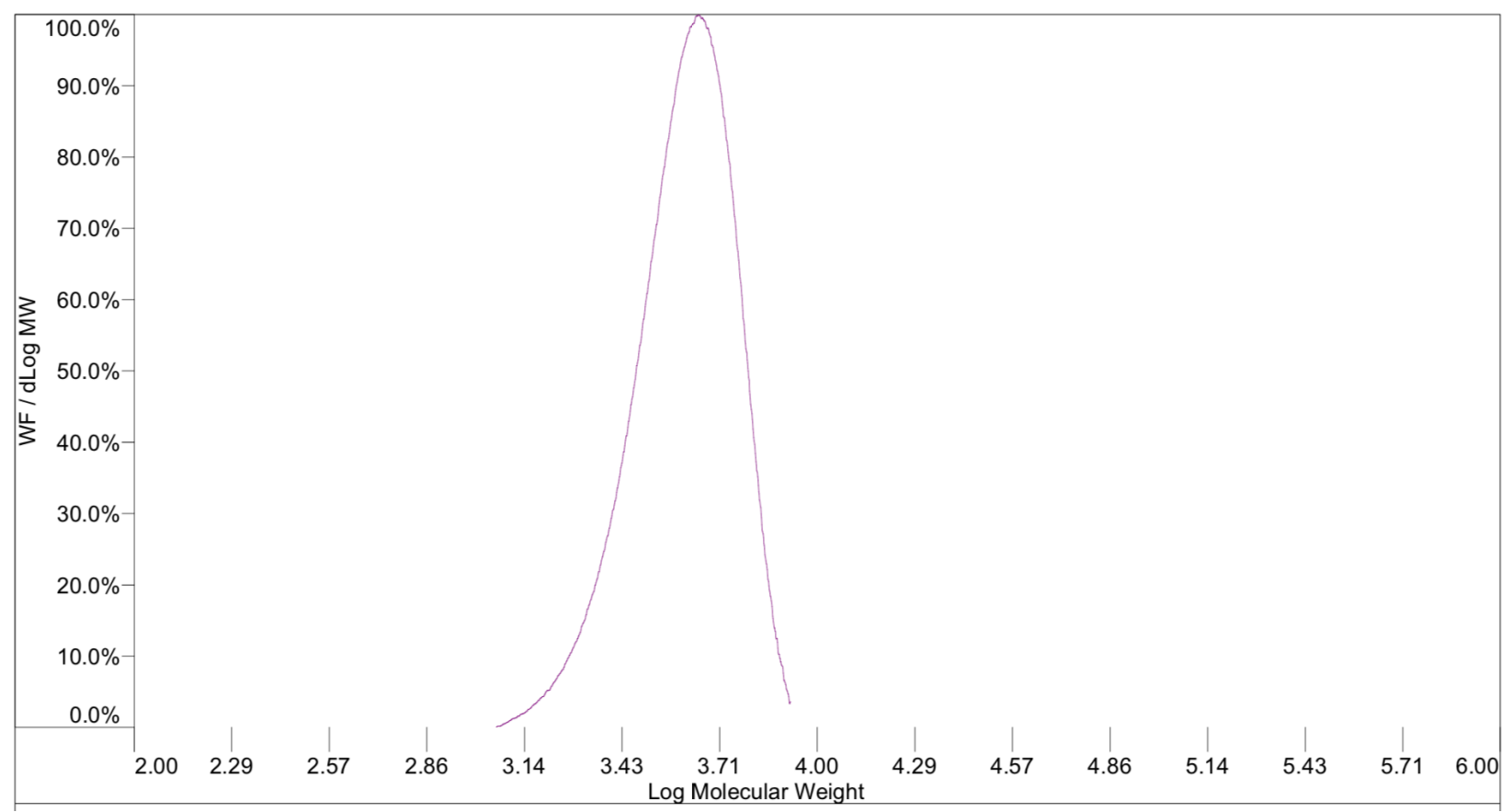

Figure S16. GPC trace of PMCP from run 3.

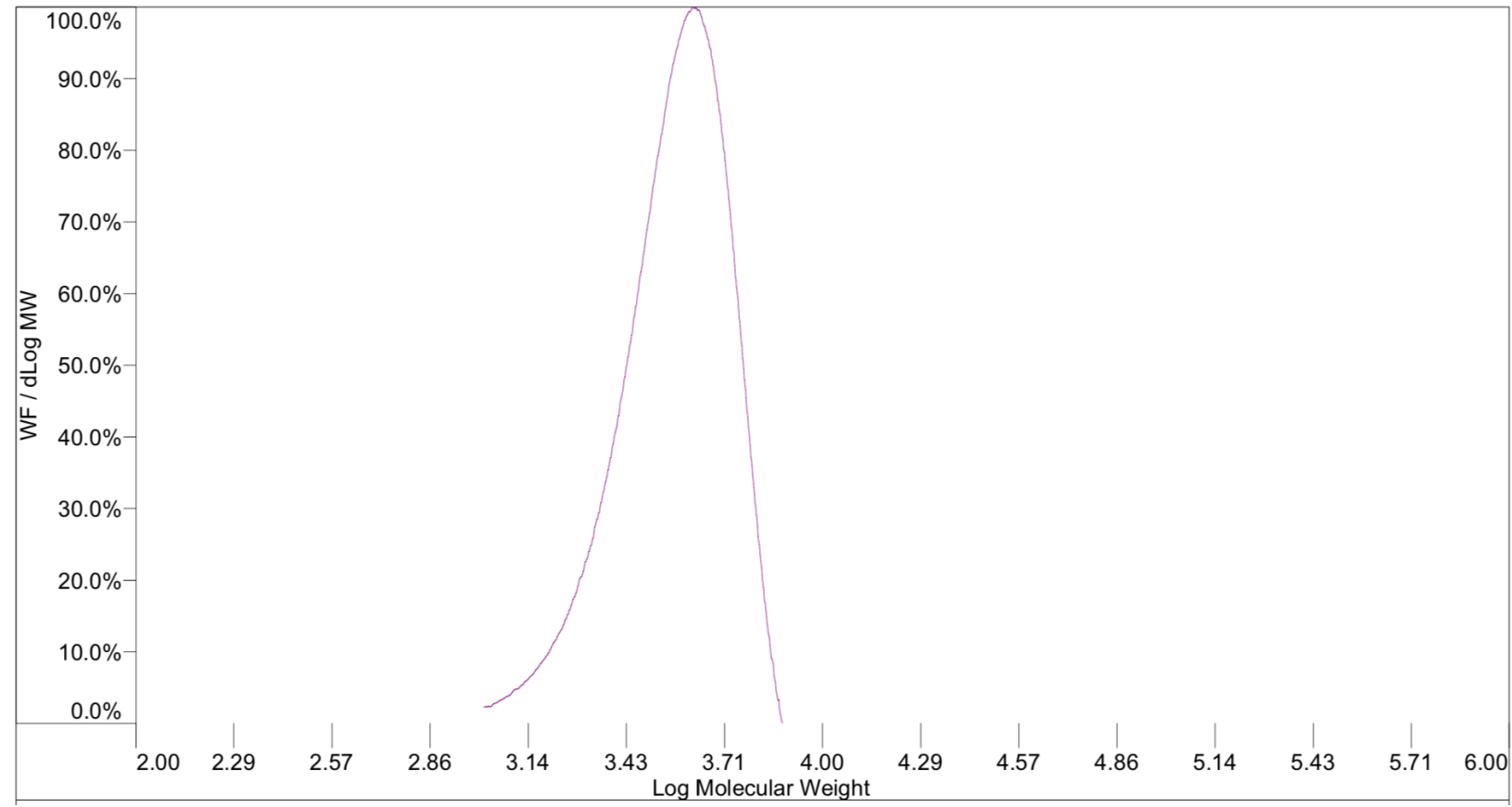

Figure S17. GPC trace of PMCP from run 4. 


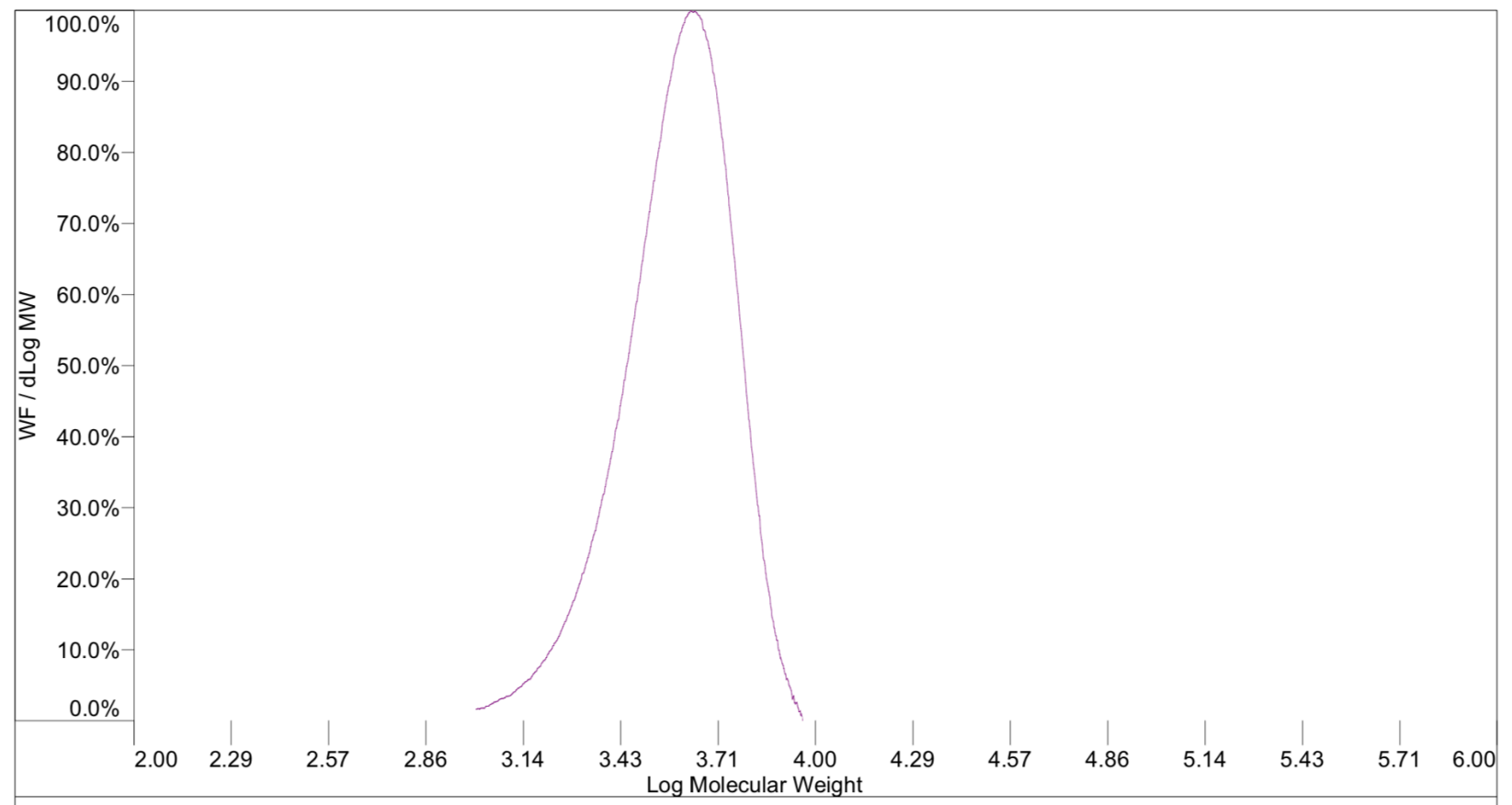

Figure S18. GPC trace of I-PMCP from run 5.

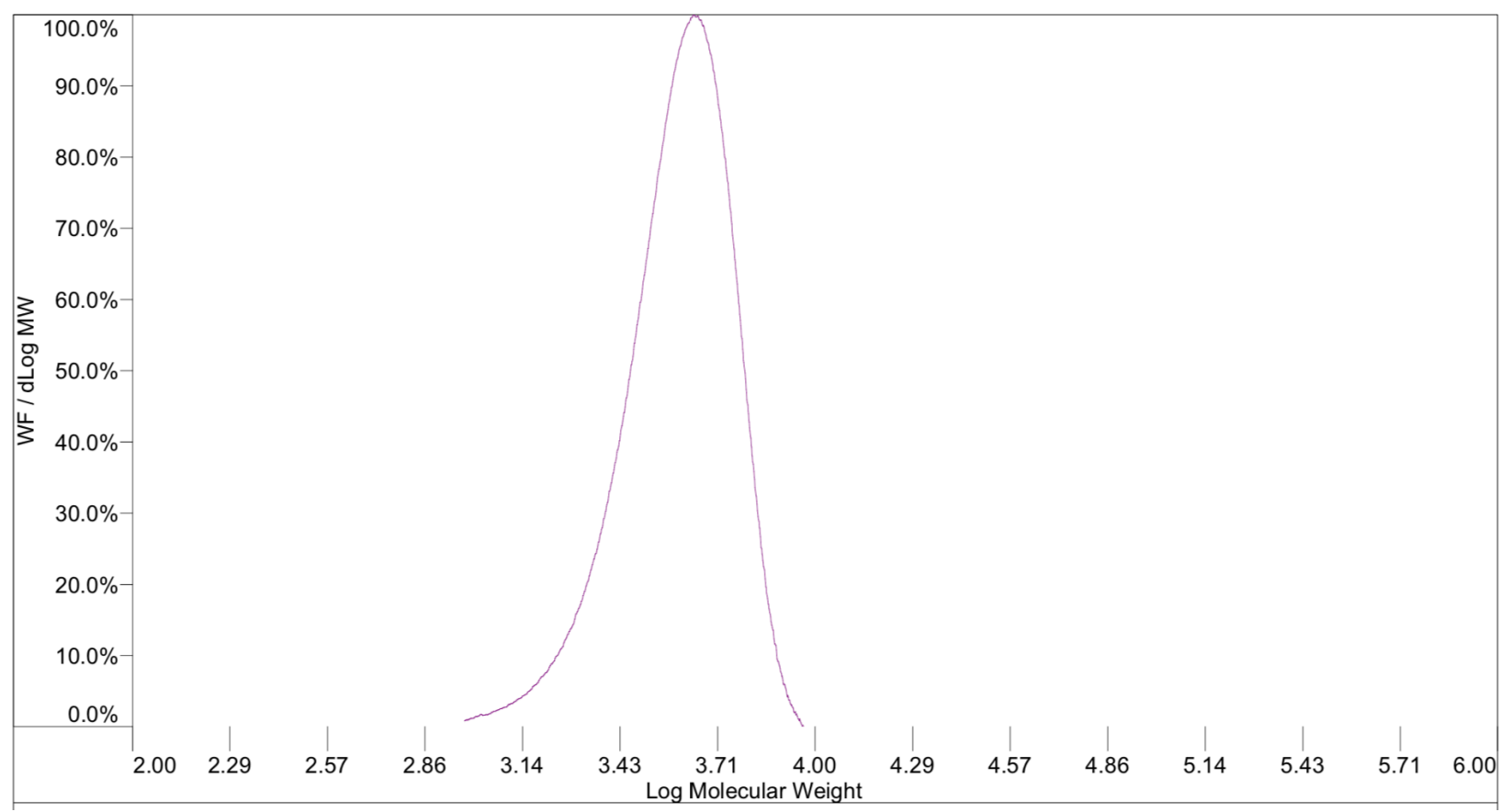

Figure S19. GPC trace of I-PMCP from run 6. 


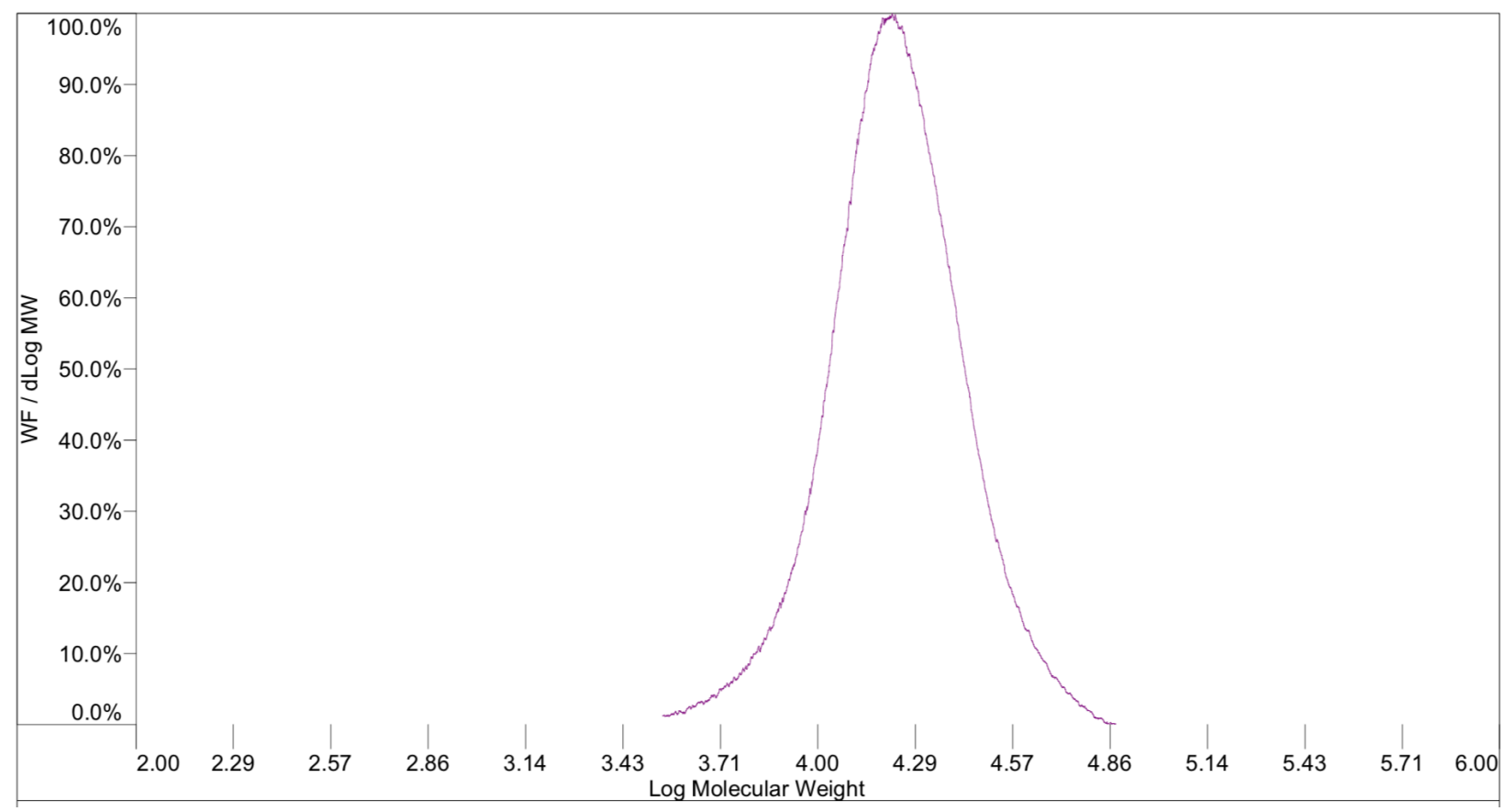

Figure S20. GPC trace of PH from run 7. 


\section{Crystallographic Information}

\section{Cp*Hf[(N-(-)-(S)-1-phenylethyl)(Nimcap $N)](M e)_{2}$}

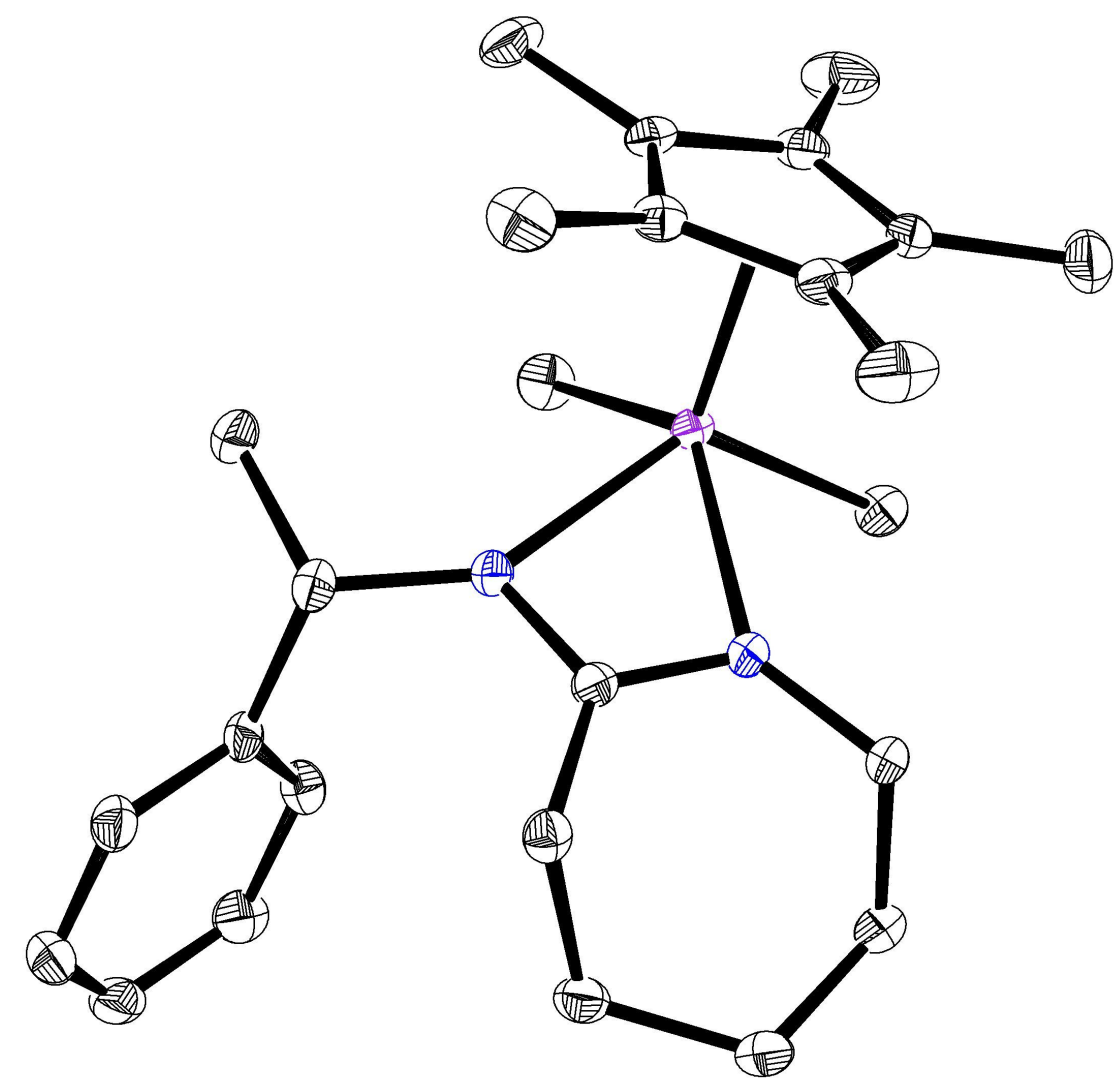

Figure S21. Crystal structure of $\left(S_{c}, S_{H f}\right)$-1 with hydrogen atoms omitted for clarity, ellipsoids for nonhydrogen atoms are shown at the $30 \%$ probability level.

Crystal Structure Collection, Structure Solution and Refinement Data for $\left(\mathbf{S}_{c}, \mathbf{S}_{H f}\right)$ 1. A suitable single crystal of $\left(S_{c}, S_{H f}\right)-1$ was selected and measured on a Bruker Smart Apex II CCD diffractometer. The crystal was kept at 150(2) K during data collection. The integral intensity was corrected for absorption using SADABS software using multi-scan method. Resulting minimum and maximum transmission are 0.307 and 0.415 respectively. The structure was solved with the ShelXT (Sheldrick, 2015a) program and refined with the ShelXL (Sheldrick, 2015c) program and least-square minimization using ShelX software package. Number of restraints used $=1$.

Crystal Data for $\left(\left(\boldsymbol{S}_{\mathrm{c}}, \boldsymbol{S}_{\boldsymbol{H f}}\right)-\mathbf{1}_{2}(M=559.09 \mathrm{~g} / \mathrm{mol})\right.$ : tetragonal, space group P4 $4_{3}$ (no. 78), $a=$ 9.4877(6) $\AA, c=27.766(2) \AA, V=2499.4(4) \AA^{3}, Z=4, T=150(2) \mathrm{K}, \mu(\mathrm{MoK \alpha})=4.187$ $\mathrm{mm}^{-1}$, Dcalc $=1.486 \mathrm{~g} / \mathrm{cm}^{3}, 33785$ reflections measured $\left(4.294^{\circ} \leq 2 \Theta \leq 62.496^{\circ}\right), 8065$ unique $\left(R_{\text {int }}=0.0216, R_{\text {sig }}=0.0201\right)$ which were used in all calculations. The final $R_{1}$ was $0.0165(I>2 \sigma(I))$ and $w R_{2}$ was 0.0378 (all data). 
Table $\mathbf{S} 1$ Crystal data and structure refinement for $\left(\mathrm{S}_{\mathrm{c}}, \mathrm{S}_{H f}\right)-1$.

\begin{tabular}{|c|c|}
\hline Identification code & UM3370R \\
\hline Empirical formula & $\mathrm{C}_{26} \mathrm{H}_{40} \mathrm{HfN}_{2}$ \\
\hline Formula weight & 559.09 \\
\hline Temperature/K & $150(2)$ \\
\hline Crystal system & tetragonal \\
\hline Space group & $\mathrm{P} 43$ \\
\hline $\mathrm{a} / \hat{A}$ & $9.4877(6)$ \\
\hline b/Å & $9.4877(6)$ \\
\hline$c / \AA$ & $27.766(2)$ \\
\hline$\alpha /^{\circ}$ & 90 \\
\hline$\beta /^{\circ}$ & 90 \\
\hline $\mathrm{y} /^{\circ}$ & 90 \\
\hline Volume/ $/ \AA^{3}$ & $2499.4(4)$ \\
\hline $\mathbf{Z}$ & 4 \\
\hline$\rho_{\text {calc }} \mathbf{g} / \mathrm{cm}^{3}$ & 1.486 \\
\hline$\mu / \mathrm{mm}^{-1}$ & 4.187 \\
\hline$F(000)$ & 1128.0 \\
\hline Crystal size/mm ${ }^{3}$ & $0.35 \times 0.22 \times 0.21$ \\
\hline Radiation & $\operatorname{MoKa}(\lambda=0.71073)$ \\
\hline 20 range for data collection $/^{\circ}$ & 4.294 to 62.496 \\
\hline Index ranges & $-13 \leq h \leq 13,-13 \leq k \leq 13,-40 \leq \mathrm{I} \leq 40$ \\
\hline Reflections collected & 33785 \\
\hline Independent reflections & $8065\left[R_{\text {int }}=0.0216, R_{\text {sigma }}=0.0201\right]$ \\
\hline Data/restraints/parameters & $8065 / 1 / 271$ \\
\hline Goodness-of-fit on $F^{2}$ & 1.063 \\
\hline Final $R$ indexes $[\mid>=2 \sigma(I)]$ & $\mathrm{R}_{1}=0.0165, w \mathrm{R}_{2}=0.0375$ \\
\hline Final $\mathbf{R}$ indexes [all data] & $R_{1}=0.0186, w R_{2}=0.0378$ \\
\hline
\end{tabular}


Table S2 Fractional Atomic Coordinates and Equivalent Isotropic Displacement Parameters $\left(\AA^{2}\right)$ for $\left(\left(S_{c}, S_{H f}\right)-1\right.$. $U_{\text {eq }}$ is defined as $1 / 3$ of of the trace of the orthogonalised $U_{I J}$ tensor.

\begin{tabular}{|c|c|c|c|c|}
\hline Atom & $x$ & $y$ & $\mathbf{z}$ & $\mathrm{U}(\mathrm{eq})$ \\
\hline $\mathrm{Hf} 1$ & $0.19076(2)$ & $0.36144(2)$ & $0.53258(2)$ & $0.02138(3)$ \\
\hline N1 & $0.1034(3)$ & $0.5289(3)$ & $0.48606(10)$ & $0.0282(6)$ \\
\hline N2 & $0.2899(2)$ & $0.5777(2)$ & $0.53028(12)$ & $0.0256(4)$ \\
\hline C1 & $0.0887(4)$ & $0.1932(4)$ & $0.59354(11)$ & $0.0305(7)$ \\
\hline $\mathrm{C} 2$ & $0.1632(3)$ & $0.2966(3)$ & $0.61990(10)$ & $0.0257(6)$ \\
\hline C3 & $0.0939(3)$ & $0.4276(3)$ & $0.61341(11)$ & $0.0266(6)$ \\
\hline $\mathrm{C} 4$ & $-0.0234(3)$ & $0.4050(4)$ & $0.58325(12)$ & $0.0309(7)$ \\
\hline C5 & $-0.0280(4)$ & $0.2615(4)$ & $0.57105(11)$ & $0.0338(7)$ \\
\hline $\mathrm{C} 6$ & $0.1233(6)$ & $0.0380(4)$ & $0.59227(16)$ & $0.0531(11)$ \\
\hline $\mathrm{C} 7$ & $0.2851(4)$ & $0.2693(5)$ & $0.65305(13)$ & $0.0423(9)$ \\
\hline C8 & $0.1273(4)$ & $0.5631(4)$ & $0.63955(14)$ & $0.0411(8)$ \\
\hline $\mathrm{C9}$ & $-0.1322(4)$ & $0.5166(5)$ & $0.57114(16)$ & $0.0516(12)$ \\
\hline C10 & $-0.1444(5)$ & $0.1895(6)$ & $0.54388(14)$ & $0.0589(13)$ \\
\hline C11 & $0.1928(3)$ & $0.6276(3)$ & $0.49992(10)$ & $0.0240(6)$ \\
\hline C12 & $0.1816(3)$ & $0.7782(3)$ & $0.48221(12)$ & $0.0276(6)$ \\
\hline C13 & $0.2081(4)$ & $0.7903(4)$ & $0.42756(12)$ & $0.0324(7)$ \\
\hline C14 & $0.0843(4)$ & $0.7457(4)$ & $0.39581(13)$ & $0.0369(8)$ \\
\hline C15 & $0.0335(4)$ & $0.5940(4)$ & $0.40206(12)$ & $0.0368(8)$ \\
\hline C16 & $-0.0128(3)$ & $0.5582(4)$ & $0.45283(12)$ & $0.0321(7)$ \\
\hline C17 & $0.4027(3)$ & $0.6687(4)$ & $0.54882(11)$ & $0.0287(6)$ \\
\hline C18 & $0.4877(4)$ & $0.5887(4)$ & $0.58661(13)$ & $0.0388(8)$ \\
\hline C19 & $0.5011(3)$ & $0.7234(3)$ & $0.50968(12)$ & $0.0268(6)$ \\
\hline C20 & $0.5426(4)$ & $0.6366(4)$ & $0.47164(13)$ & $0.0315(7)$ \\
\hline $\mathrm{C} 21$ & $0.6337(3)$ & $0.6879(4)$ & $0.43590(14)$ & $0.0372(8)$ \\
\hline $\mathrm{C} 22$ & $0.6832(4)$ & $0.8250(5)$ & $0.43883(16)$ & $0.0443(10)$ \\
\hline C23 & $0.6422(4)$ & $0.9108(4)$ & $0.47644(16)$ & $0.0446(9)$ \\
\hline $\mathrm{C} 24$ & $0.5532(4)$ & $0.8594(4)$ & $0.51165(13)$ & $0.0355(7)$ \\
\hline $\mathrm{C} 25$ & $0.1308(4)$ & $0.2152(4)$ & $0.47069(13)$ & $0.0333(7)$ \\
\hline C26 & $0.4039(3)$ & $0.2548(4)$ & $0.53572(18)$ & $0.0384(7)$ \\
\hline
\end{tabular}


Table S3 Anisotropic Displacement Parameters $\left(\AA^{2}\right)$ for $\left(\left(S_{c}, S_{H f}\right)-1\right.$. The Anisotropic displacement factor exponent takes the form: -

$$
2 \pi^{2}\left[h^{2} a * 2 U_{11}+2 h k a * b^{*} U_{12}+\ldots\right] \text {. }
$$

\begin{tabular}{|c|c|c|c|c|c|c|}
\hline Atom & $\mathrm{U}_{11}$ & $\mathbf{U}_{22}$ & $\mathrm{U}_{33}$ & $\mathbf{U}_{23}$ & $\mathrm{U}_{13}$ & $U_{12}$ \\
\hline Hf1 & $0.01976(6)$ & $.02422(6)$ & $.02015(5)$ & $0.00091(5)$ & $0.00087(5)$ & $0.00320(4)$ \\
\hline N1 & $0.0256(13)$ & $0.0300(14)$ & $0.0291(13)$ & $0.0076(11)$ & $-0.0075(10)$ & $-0.0053(11)$ \\
\hline N2 & $0.0204(10)$ & $0.0305(11)$ & $0.0259(10)$ & $0.0030(12)$ & $-0.0014(12)$ & $-0.0020(9)$ \\
\hline C1 & $0.0423(19)$ & $0.0266(16)$ & $0.0225(14)$ & $0.0025(12)$ & 0.004 & $-0.0041(14)$ \\
\hline $\mathrm{C} 2$ & $0.0281(15)$ & $0.0307(16)$ & $0.0183(12)$ & $0.0035(11)$ & $0.0022(10)$ & $0.0057(12)$ \\
\hline $\mathrm{C3}$ & $0.0265(15)$ & $0.0288(15)$ & $0.0244(13)$ & $-0.0004(11)$ & $0.0062(11)$ & $0.0050(12)$ \\
\hline C4 & $0.0195(14)$ & $0.0453(19)$ & $0.0278(15)$ & $0.0085(14)$ & $0.0065(11)$ & $0.0073(13)$ \\
\hline C5 & $0.0307(17)$ & $0.048(2)$ & $0.0225(14)$ & $0.0047(13)$ & $0.0010(12)$ & $-0.0129(15)$ \\
\hline C6 & $0.089(4)$ & $0.0274(18)$ & $0.043(2)$ & 0.0 & 0.0 & (2) \\
\hline$C 7$ & $0.0373(19)$ & $0.062(3)$ & $0.0274(16)$ & $0.0087(16)$ & $-0.0063(14)$ & $0.0094(18)$ \\
\hline 58 & $0.052(2)$ & $0.0342(18)$ & $0.0366(18)$ & $-0.0102(15)$ & $0.0114(16)$ & $0.0004(16)$ \\
\hline 9 & $0.036(2)$ & $0.072(3)$ & $0.047(2)$ & $0.019(2)$ & 17) & $0.027(2)$ \\
\hline C10 & $0.045(2)$ & $0.096(4)$ & $0.035(2)$ & $-0.004(2)$ & -0.00 & $-0.035(2)$ \\
\hline 11 & $0.0198(13)$ & $0.0294(15)$ & $0.0226(12)$ & 0.00 & 0.00 & $0.0019(11)$ \\
\hline 12 & $0.0237(14)$ & $0.0254(15)$ & $0.0336(15)$ & $-0.0003(12)$ & -0.0 & $0.0027(11)$ \\
\hline$C 13$ & $0.0332(17)$ & $0.0303(16)$ & $0.0337(16)$ & $0.0088(13)$ & 0.00 & $0.0001(13)$ \\
\hline 14 & $0.0388(18)$ & $0.0401(19)$ & $0.0317(16)$ & $0.0124(14)$ & -0.0 & $-0.0016(16)$ \\
\hline C15 & $0.0394(19)$ & $0.043(2)$ & $0.0283(15)$ & 0.00 & -0.0 & $63(15)$ \\
\hline 16 & $0.0249(15)$ & $0.0393(18)$ & $0.0321(16)$ & $0.0100(13)$ & $-0.0097(13)$ & $-0.0059(13)$ \\
\hline C17 & $0.0242(14)$ & $0.0333(16)$ & $0.0286(14)$ & $-0.0034(12)$ & $-0.0045(11)$ & $-0.0008(12)$ \\
\hline 18 & $0.0288(17)$ & $0.053(2)$ & $0.0345(17)$ & $0.0069(16)$ & $-0.0091(14)$ & $-0.0061(16)$ \\
\hline 19 & $0.0184(13)$ & $0.0299(15)$ & $0.0320(14)$ & $0.0010(12)$ & $-0.0054(11)$ & $-0.0024(11)$ \\
\hline $\mathrm{C} 20$ & $0.0244(15)$ & $0.0314(17)$ & $0.0388(17)$ & $-0.0029(13)$ & $0.0001(13)$ & $-0.0027(12)$ \\
\hline $\mathrm{C} 21$ & $0.0279(17)$ & $0.046(2)$ & $0.0373(18)$ & $0.0050(15)$ & $0.0030(13)$ & $0.0050(15)$ \\
\hline $\mathrm{C} 22$ & $0.0277(17)$ & $0.058(2)$ & $0.047(2)$ & $0.0239(19)$ & $-0.0061(15)$ & $-0.0057(16)$ \\
\hline $\mathrm{C} 23$ & $0.044(2)$ & $0.037(2)$ & $0.052(2)$ & $0.0133(17)$ & $-0.0147(18)$ & $-0.0141(17)$ \\
\hline 24 & $0.0391(19)$ & $0.0299(16)$ & $0.0376(17)$ & $-0.0015(13)$ & $-0.0113(14)$ & $-0.0065(14)$ \\
\hline $\mathrm{C} 25$ & $0.0354(18)$ & $0.0353(18)$ & $0.0290(15)$ & $-0.0048(13)$ & $0.0014(13)$ & $0.0042(15)$ \\
\hline C26 & $0.0317(15)$ & $0.0381(17)$ & $0.0453(18)$ & $0.0008(19)$ & $0.0024(18)$ & $0.0116(13)$ \\
\hline
\end{tabular}


Table S4 Bond Lengths for $\left(\mathrm{S}_{\mathrm{c}}, \mathrm{S}_{H f}\right)-1$.

\begin{tabular}{llllll}
\multicolumn{5}{c}{ Atom Atom Length/A } & \multicolumn{3}{l}{ Atom Atom Length/A } \\
$\mathrm{Hf} 1$ & $\mathrm{~N} 1$ & $2.209(3)$ & $\mathrm{C} 3$ & $\mathrm{C} 4$ & $1.409(5)$ \\
$\mathrm{Hf} 1$ & $\mathrm{~N} 2$ & $2.258(2)$ & $\mathrm{C} 3$ & $\mathrm{C} 8$ & $1.509(5)$ \\
$\mathrm{Hf} 1$ & $\mathrm{C} 26$ & $2.263(3)$ & $\mathrm{C} 4$ & $\mathrm{C} 5$ & $1.404(5)$ \\
$\mathrm{Hf} 1$ & $\mathrm{C} 25$ & $2.281(3)$ & $\mathrm{C} 4$ & $\mathrm{C} 9$ & $1.517(5)$ \\
$\mathrm{Hf} 1$ & $\mathrm{C} 3$ & $2.505(3)$ & $\mathrm{C} 5$ & $\mathrm{C} 10$ & $1.502(5)$ \\
$\mathrm{Hf} 1$ & $\mathrm{C} 4$ & $2.505(3)$ & $\mathrm{C} 11$ & $\mathrm{C} 12$ & $1.515(4)$ \\
$\mathrm{Hf} 1$ & $\mathrm{C} 2$ & $2.515(3)$ & $\mathrm{C} 12$ & $\mathrm{C} 13$ & $1.543(5)$ \\
$\mathrm{Hf} 1$ & $\mathrm{C} 5$ & $2.519(3)$ & $\mathrm{C} 13$ & $\mathrm{C} 14$ & $1.529(5)$ \\
$\mathrm{Hf} 1$ & $\mathrm{C} 1$ & $2.520(3)$ & $\mathrm{C} 14$ & $\mathrm{C} 15$ & $1.528(5)$ \\
$\mathrm{Hf} 1$ & $\mathrm{C} 11$ & $2.684(3)$ & $\mathrm{C} 15$ & $\mathrm{C} 16$ & $1.515(5)$ \\
$\mathrm{N} 1$ & $\mathrm{C} 11$ & $1.321(4)$ & $\mathrm{C} 17$ & $\mathrm{C} 19$ & $1.524(4)$ \\
$\mathrm{N} 1$ & $\mathrm{C} 16$ & $1.465(4)$ & $\mathrm{C} 17$ & $\mathrm{C} 18$ & $1.526(5)$ \\
$\mathrm{N} 2$ & $\mathrm{C} 11$ & $1.335(4)$ & $\mathrm{C} 19$ & $\mathrm{C} 24$ & $1.382(5)$ \\
$\mathrm{N} 2$ & $\mathrm{C} 17$ & $1.468(4)$ & $\mathrm{C} 19$ & $\mathrm{C} 20$ & $1.396(5)$ \\
$\mathrm{C} 1$ & $\mathrm{C} 2$ & $1.414(5)$ & $\mathrm{C} 20$ & $\mathrm{C} 21$ & $1.403(5)$ \\
$\mathrm{C} 1$ & $\mathrm{C} 5$ & $1.426(5)$ & $\mathrm{C} 21$ & $\mathrm{C} 22$ & $1.385(6)$ \\
$\mathrm{C} 1$ & $\mathrm{C} 6$ & $1.509(5)$ & $\mathrm{C} 22$ & $\mathrm{C} 23$ & $1.380(7)$ \\
$\mathrm{C} 2$ & $\mathrm{C} 3$ & $1.417(4)$ & $\mathrm{C} 23$ & $\mathrm{C} 24$ & $1.381(6)$ \\
$\mathrm{C} 2$ & $\mathrm{C} 7$ & $1.500(4)$ & & &
\end{tabular}

Table S5 Bond Angles for $\left(\mathrm{S}_{\mathrm{c}}, \mathrm{S}_{H f}\right)-1$.

Atom Atom Atom Angle ${ }^{\circ}$ Atom Atom Atom Angle ${ }^{\circ}$

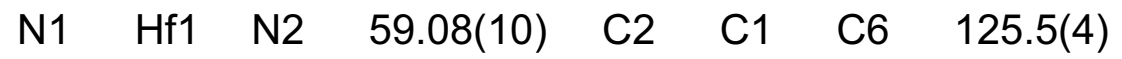

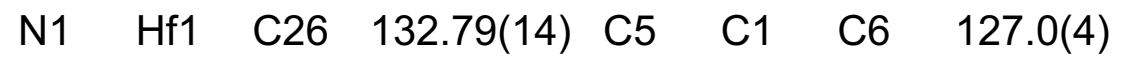

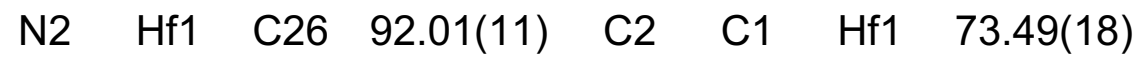

$\mathrm{N} 1 \quad \mathrm{Hf} 1 \quad \mathrm{C} 25 \quad 84.46(12) \quad \mathrm{C} 5 \quad \mathrm{C} 1 \quad \mathrm{Hf} 1 \quad 73.54(18)$

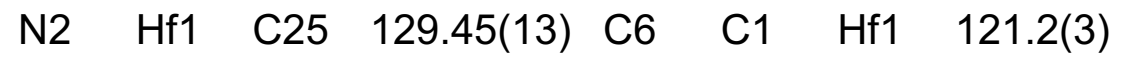

C26 Hf1 C25 88.85(15) C1 $\quad$ C2 $\quad$ C3 $108.1(3)$

N1 Hf1 C3 $101.89(11)$ C1 $\mathrm{C} 2 \quad \mathrm{C} 7 \quad 125.7(3)$

N2 $\mathrm{Hf} 1 \quad \mathrm{C} 3 \quad 87.16(11) \quad \mathrm{C} 3 \quad \mathrm{C} 2 \quad \mathrm{C} 7 \quad 126.0(3)$

C26 Hf1 C3 $113.91(15)$ C1 $\mathrm{C} 2$ Hf1 $73.89(17)$

C25 Hf1 C3 137.39(12) $\mathrm{C} 3$ C2 Hf1 $73.22(16)$ 


\begin{tabular}{|c|c|c|c|c|c|c|c|}
\hline 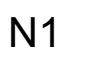 & ff1 & 4 & $34.59(11)$ & $\mathrm{C7}$ & $\mathrm{C} 2$ & $\mathrm{Hf} 1$ & \\
\hline ? & ff1 & 4 & $01.76(11)$ & $\mathrm{C} 4$ & $\mathrm{C} 3$ & $\mathrm{C} 2$ & $08.0(3)$ \\
\hline 26 & 1 & 4 & 4) & C4 & 3 & 8 & (3) \\
\hline 25 & $\mathrm{f} 1$ & 1 & 3) & $\mathrm{C} 2$ & 3 & 58 & $6.0(3)$ \\
\hline & $\mathrm{f} 1$ & 4 & 2.67 & $\mathrm{C} 4$ & C3 & $\mathrm{Hf} 1$ & $3.68(18)$ \\
\hline & f1 & - & 0) & $\mathrm{C} 2$ & C3 & $\mathrm{Hf} 1$ & (16) \\
\hline 2 & f1 & $\mathrm{C} 2$ & 07.0 & C8 & C3 & Hf1 & $24.6(2)$ \\
\hline 26 & f1 & 2 & 6 & 5 & 4 & 3 & $08.4(3)$ \\
\hline 25 & If1 & C2 & 23. & C5 & C4 & C9 & 127.0(4) \\
\hline 3 & $\mathrm{Hf} 1$ & $\mathrm{C} 2$ & 32.8 & $\mathrm{C} 3$ & $\mathrm{C} 4$ & 9 & $24.3(4)$ \\
\hline 4 & $f 1$ & 2 & 4.1 & C5 & C4 & $\mathrm{Hf} 1$ & $4.33(19)$ \\
\hline 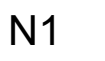 & If1 & C5 & 102. & C3 & C4 & $\mathrm{Hf} 1$ & $3.65(17)$ \\
\hline & f1 & 5 & 1) & C9 & $\mathrm{C} 4$ & $\mathrm{ff} 1$ & $122.9(2)$ \\
\hline 26 & ff1 & C5 & 123. & $\mathrm{C} 4$ & C5 & $\mathrm{C} 1$ & $108.1(3)$ \\
\hline 25 & If1 & C5 & 83.3 & C4 & C5 & C10 & $25.8(4)$ \\
\hline 3 & If1 & 5 & ) & 1 & 5 & 10 & \\
\hline 4 & 1 & $\mathrm{C} 5$ & 32. & $\mathrm{C} 4$ & C5 & f1 & 3.2 \\
\hline $\mathrm{C} 2$ & 1 & 5 & 5 & C1 & C5 & If1 & 19) \\
\hline 1 & $\mathrm{f} 1$ & 1 & 2) & C10 & $\mathrm{C} 5$ & $\mathrm{Hf} 1$ & $24.3(2)$ \\
\hline 2 & ff1 & C1 & 138 & N1 & C11 & 12 & $112.0(3)$ \\
\hline 26 & $\mathrm{f} 1$ & $\mathrm{C} 1$ & 10 & N1 & $\mathrm{C} 1$ & $\mathrm{C} 12$ & $122.0(3)$ \\
\hline 25 & ff1 & $\mathrm{C} 1$ & 12 & N2 & C11 & $\mathrm{C} 12$ & $126.0(3)$ \\
\hline 3 & $\mathrm{Hf} 1$ & $\mathrm{C} 1$ & 1.2 & $\mathrm{~N} 1$ & C11 & $\mathrm{Hf} 1$ & 54.9 \\
\hline 4 & $\mathrm{Hf} 1$ & 1 & ) & 2 & 11 & f1 & $7.16(15)$ \\
\hline 2 & ff1 & C1 & & C12 & C11 & $\mathrm{Hf1}$ & $175.5(2)$ \\
\hline 5 & ff1 & $\mathrm{C} 1$ & 32.8 & C11 & $\mathrm{C} 12$ & $\mathrm{C} 13$ & $112.2(3)$ \\
\hline 1 & f1 & C11 & 29.3 & 14 & 13 & 12 & $14.9(3)$ \\
\hline 2 & ff1 & C11 & 29.7 & C15 & C14 & C13 & $116.0(3)$ \\
\hline 26 & $f f 1$ & C11 & 115 & C16 & C15 & C14 & $114.1(3)$ \\
\hline $\mathrm{C} 25$ & $\mathrm{Hf} 1$ & C11 & 108. & N1 & C16 & C15 & $114.2(3)$ \\
\hline 3 & $\mathrm{Hf} 1$ & C11 & 93.95 & N2 & C17 & C19 & $113.4(3)$ \\
\hline $\mathrm{C4}$ & $\mathrm{Hf} 1$ & C11 & 92.3 & 12 & C17 & 18 & $09.5(3)$ \\
\hline 2 & 1 & C11 & 123 & C19 & C17 & C18 & 109.6(3) \\
\hline 65 & $\mathrm{Hf1}$ & C11 & 120. & $\mathrm{C} 24$ & C19 & $\mathrm{C} 20$ & 118.7(3) \\
\hline C1 & $\mathrm{Hf} 1$ & C11 & 145. & $\mathrm{C} 24$ & C19 & C17 & $120.6(3)$ \\
\hline C11 & N1 & & 122. & $\mathrm{C} 20$ & C19 & C17 & $120.7(3)$ \\
\hline C11 & N1 & $\mathrm{Hf} 1$ & 95.67 & C19 & $\mathrm{C} 20$ & $\mathrm{C} 21$ & $120.3(3)$ \\
\hline C16 & N1 & $\mathrm{Hf} 1$ & $142.0(2)$ & $\mathrm{C} 22$ & $\mathrm{C} 21$ & $\mathrm{C} 20$ & $119.5(4)$ \\
\hline
\end{tabular}




$\begin{array}{llllllll}\mathrm{C} 11 & \mathrm{~N} 2 & \mathrm{C} 17 & 121.0(3) & \mathrm{C} 23 & \mathrm{C} 22 & \mathrm{C} 21 & 120.2(4) \\ \mathrm{C} 11 & \mathrm{~N} 2 & \mathrm{Hf} 1 & 93.04(18) & \mathrm{C} 22 & \mathrm{C} 23 & \mathrm{C} 24 & 120.0(4) \\ \mathrm{C} 17 & \mathrm{~N} 2 & \mathrm{Hf} 1 & 145.9(2) & \mathrm{C} 23 & \mathrm{C} 24 & \mathrm{C} 19 & 121.4(4) \\ \mathrm{C} 2 & \mathrm{C} 1 & \mathrm{C} 5 & 107.4(3) & & & & \end{array}$

Table S6 Hydrogen Atom Coordinates and Isotropic Displacement Parameters $\left(\AA^{2}\right)$ for $\left(S_{c}, S_{H f}\right)-1$.

\begin{tabular}{lllll}
\multicolumn{1}{rr}{ Atom } & \multicolumn{1}{c}{$\boldsymbol{x}$} & \multicolumn{1}{c}{$\boldsymbol{y}$} & \multicolumn{1}{c}{$\boldsymbol{z}$} & \multicolumn{1}{c}{ U(eq) } \\
H6A & 0.086297 & -0.007575 & 0.621308 & 0.08 \\
H6B & 0.22578 & 0.025672 & 0.590955 & 0.08 \\
H6C & 0.080304 & -0.004869 & 0.563715 & 0.08 \\
H7A & 0.249511 & 0.241268 & 0.684778 & 0.063 \\
H7B & 0.341647 & 0.355185 & 0.65622 & 0.063 \\
H7C & 0.343579 & 0.193506 & 0.639807 & 0.063 \\
H8A & 0.119729 & 0.64245 & 0.617108 & 0.062 \\
H8B & 0.223353 & 0.55858 & 0.65242 & 0.062 \\
H8C & 0.060425 & 0.576135 & 0.666076 & 0.062 \\
H9A & -0.182438 & 0.489558 & 0.541722 & 0.077 \\
H9B & -0.084952 & 0.607268 & 0.566105 & 0.077 \\
H9C & -0.199449 & 0.525068 & 0.597766 & 0.077 \\
H10A & -0.216353 & 0.156726 & 0.566596 & 0.088 \\
H10B & -0.106024 & 0.108846 & 0.526185 & 0.088 \\
H10C & -0.186904 & 0.256124 & 0.521131 & 0.088 \\
H12A & 0.251108 & 0.837206 & 0.499573 & 0.033 \\
H12B & 0.08641 & 0.814969 & 0.489664 & 0.033 \\
H13A & 0.232256 & 0.889405 & 0.419965 & 0.039 \\
H13B & 0.290846 & 0.731614 & 0.419204 & 0.039 \\
H14A & 0.004153 & 0.809632 & 0.402405 & 0.044 \\
H14B & 0.111696 & 0.759226 & 0.361715 & 0.044 \\
H15A & -0.0465 & 0.577419 & 0.379867 & 0.044 \\
H15B & 0.110537 & 0.529288 & 0.392634 & 0.044 \\
H16A & -0.06874 & 0.637854 & 0.465714 & 0.038 \\
H16B & -0.075304 & 0.474711 & 0.451587 & 0.038 \\
H1 & 0.357927 & 0.75173 & 0.564879 & 0.034 \\
H18A & 0.424944 & 0.557697 & 0.61256 & 0.058 \\
H18B & 0.560723 & 0.650621 & 0.599865 & 0.058
\end{tabular}




$\begin{array}{lllll}\text { H18C } & 0.532165 & 0.506285 & 0.571682 & 0.058 \\ \text { H2O } & 0.50894 & 0.542414 & 0.469938 & 0.038 \\ \text { H21 } & 0.661333 & 0.628974 & 0.409886 & 0.045 \\ \text { H22 } & 0.745428 & 0.860081 & 0.414869 & 0.053 \\ \text { H23 } & 0.675306 & 1.005183 & 0.478121 & 0.054 \\ \text { H24 } & 0.527201 & 0.918635 & 0.537753 & 0.043 \\ \text { H25A } & 0.032452 & 0.231998 & 0.461553 & 0.05 \\ \text { H25B } & 0.142029 & 0.116945 & 0.480903 & 0.05 \\ \text { H25C } & 0.192148 & 0.233739 & 0.443009 & 0.05 \\ \text { H26A } & 0.461921 & 0.287016 & 0.508671 & 0.058 \\ \text { H26B } & 0.39147 & 0.152471 & 0.533746 & 0.058 \\ \text { H26C } & 0.450613 & 0.278894 & 0.56611 & 0.058\end{array}$




\section{Cp*Hf[(N-(+)-(R)-1-phenylethyl)(Nimcap $N)](M e)_{2}$}

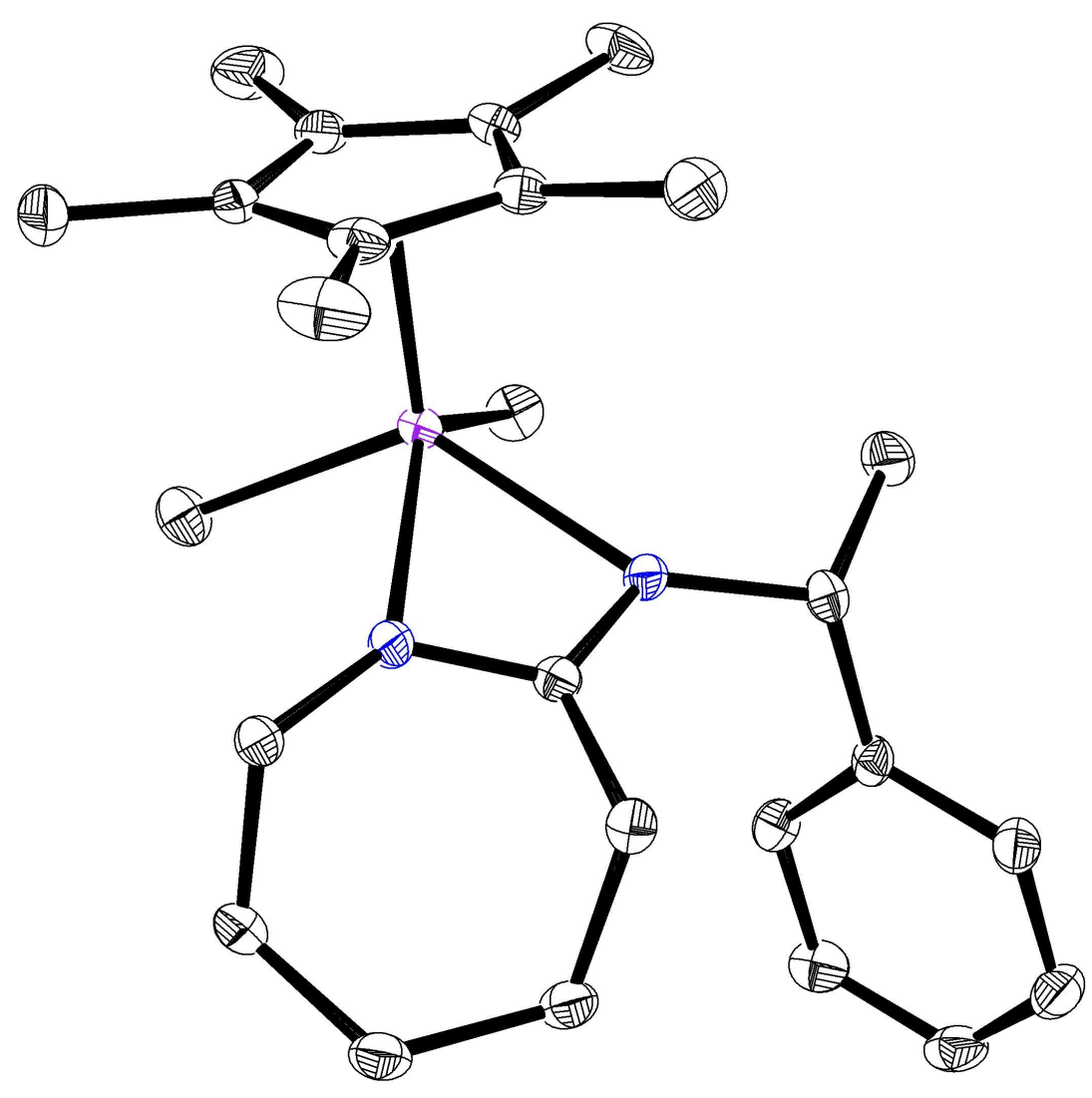

Figure S22. Crystal structure of $\left(\boldsymbol{R}_{\mathrm{c}}, \boldsymbol{R}_{\boldsymbol{H}}\right)$-1 with hydrogen atoms omitted for clarity, ellipsoids for nonhydrogen atoms are shown at the $30 \%$ probability level.

Crystal Structure Collection, Structure Solution and Refinement Data for $\left(\boldsymbol{R}_{\mathrm{c}}, \boldsymbol{R}_{H f}\right)$ 1. A suitable single crystal of $\left(\boldsymbol{R}_{\mathrm{c}}, \boldsymbol{R}_{\boldsymbol{H f}}\right)-1$ was selected and measured on a Bruker Smart Apex II CCD diffractometer. The crystal was kept at 150(2) K during data collection. The integral intensity was corrected for absorption using SADABS software using multi-scan method. Resulting minimum and maximum transmission are 0.360 and 0.451 respectively. The structure was solved with the ShelXT (Sheldrick, 2015a) program and refined with the ShelXL (Sheldrick, 2015c) program and least-square minimization using ShelX software package. Number of restraints used $=1$.

Crystal Data for $\left(\boldsymbol{R}_{\mathrm{c}}, \boldsymbol{R}_{\boldsymbol{H f}}\right)-\mathbf{1}(M=559.09 \mathrm{~g} / \mathrm{mol})$ : tetragonal, space group P41 (no. 76), $a=$ 9.4910(4) $\AA, c=27.7364(11) \AA, V=2498.5(2) \AA^{3}, Z=4, T=150(2) \mathrm{K}, \mu(\mathrm{MoK \alpha})=4.189$ $\mathrm{mm}^{-1}$, Dcalc $=1.486 \mathrm{~g} / \mathrm{cm}^{3}, 44784$ reflections measured $\left(4.292^{\circ} \leq 2 \Theta \leq 62.496^{\circ}\right), 8106$ unique $\left(R_{\text {int }}=0.0207, R_{\text {sig }}=0.0173\right)$ which were used in all calculations. The final $R_{1}$ was $0.0181(\mathrm{I}>2 \sigma(\mathrm{I}))$ and $w R_{2}$ was 0.0429 (all data). 
Table S7 Crystal data and structure refinement for $\left(\boldsymbol{R}_{\mathrm{c}}, \boldsymbol{R}_{H f}\right)-1$.

\begin{tabular}{|c|c|}
\hline Identification code & UM3379R \\
\hline Empirical formula & $\mathrm{C} 26 \mathrm{H} 40 \mathrm{HfN} 2$ \\
\hline Formula weight & 559.09 \\
\hline Temperature/K & $150(2)$ \\
\hline Crystal system & tetragonal \\
\hline Space group & $\mathrm{P} 41$ \\
\hline $\mathrm{a} / \AA \AA \AA$ & $9.4910(4)$ \\
\hline b/Å & $9.4910(4)$ \\
\hline c/Å & $27.7364(11)$ \\
\hline$\alpha /^{\circ}$ & 90 \\
\hline$\beta /^{\circ}$ & 90 \\
\hline $\mathrm{y} /{ }^{\circ}$ & 90 \\
\hline Volume/Å3 & $2498.5(2)$ \\
\hline $\mathbf{z}$ & 4 \\
\hline$\rho c a l c g / c m 3$ & 1.486 \\
\hline$\mu / m m-1$ & 4.189 \\
\hline$F(000)$ & 1128.0 \\
\hline Crystal size/mm3 & $0.38 \times 0.24 \times 0.19$ \\
\hline Radiation & $\operatorname{MoKa}(\lambda=0.71073)$ \\
\hline $2 \Theta$ range for data collection $/{ }^{\circ}$ & 4.292 to 62.496 \\
\hline Index ranges & $-13 \leq h \leq 13,-13 \leq k \leq 13,-40 \leq \mathrm{I} \leq 40$ \\
\hline Reflections collected & 44784 \\
\hline Independent reflections & $8106[$ Rint $=0.0207$, Rsigma $=0.0173]$ \\
\hline Data/restraints/parameters & $8106 / 1 / 270$ \\
\hline Goodness-of-fit on F2 & 1.070 \\
\hline Final $R$ indexes $[\mid>=2 \sigma(I)]$ & $\mathrm{R} 1=0.0181, w R 2=0.0426$ \\
\hline Final $R$ indexes [all data] & $\mathrm{R} 1=0.0199, \mathrm{wR} 2=0.0429$ \\
\hline Largest diff. peak/hole / e Â-3 & $1.10 /-1.00$ \\
\hline
\end{tabular}


Table S8 Fractional Atomic Coordinates and Equivalent Isotropic Displacement Parameters $\left(\AA^{2}\right)$ for $\left(R_{\mathrm{c}}, R_{H f}\right)-1$. $U_{\text {eq }}$ is defined as $1 / 3$ of of the trace of the orthogonalised $U_{I J}$ tensor.

\begin{tabular}{|c|c|c|c|c|}
\hline Atom & $x$ & $y$ & $z$ & $\mathrm{U}(\mathrm{eq})$ \\
\hline $\mathrm{Hf1}$ & $0.36187(2)$ & $0.19119(2)$ & $0.50889(2)$ & $0.02040(3)$ \\
\hline N1 & $0.5293(3)$ & $0.1038(3)$ & $0.46228(11)$ & $0.0273(6)$ \\
\hline N2 & $0.5787(3)$ & $0.2901(2)$ & $0.50646(13)$ & $0.0241(5)$ \\
\hline C1 & $0.1930(4)$ & $0.0891(4)$ & $0.56995(12)$ & $0.0294(7)$ \\
\hline $\mathrm{C} 2$ & $0.2969(4)$ & $0.1642(3)$ & $0.59651(11)$ & $0.0248(6)$ \\
\hline C3 & $0.4276(4)$ & $0.0943(4)$ & $0.58981(11)$ & $0.0260(6)$ \\
\hline C4 & $0.4051(4)$ & $-0.0231(4)$ & $0.55929(13)$ & $0.0299(7)$ \\
\hline C5 & $0.2610(4)$ & $-0.0272(4)$ & $0.54735(12)$ & $0.0315(7)$ \\
\hline C6 & $0.0386(5)$ & $0.1244(6)$ & $0.56857(17)$ & $0.0515(12)$ \\
\hline $\mathrm{C7}$ & $0.2695(5)$ & $0.2867(4)$ & $0.62937(13)$ & $0.0406(9)$ \\
\hline $\mathrm{C} 8$ & $0.5631(4)$ & $0.1279(5)$ & $0.61598(15)$ & $0.0396(9)$ \\
\hline C9 & $0.5154(6)$ & $-0.1323(5)$ & $0.54762(17)$ & $0.0493(12)$ \\
\hline C10 & $0.1899(7)$ & $-0.1437(5)$ & $0.52037(15)$ & $0.0555(13)$ \\
\hline C11 & $0.6286(3)$ & $0.1931(3)$ & $0.47617(11)$ & $0.0223(6)$ \\
\hline C12 & $0.7787(4)$ & $0.1817(4)$ & $0.45837(13)$ & $0.0263(6)$ \\
\hline C13 & $0.7907(4)$ & $0.2081(4)$ & $0.40396(13)$ & $0.0310(7)$ \\
\hline C14 & $0.7458(4)$ & $0.0847(4)$ & $0.37201(14)$ & $0.0356(8)$ \\
\hline C15 & $0.5942(4)$ & $0.0344(4)$ & $0.37826(13)$ & $0.0344(8)$ \\
\hline C16 & $0.5587(4)$ & $-0.0126(4)$ & $0.42937(13)$ & $0.0310(7)$ \\
\hline C17 & $0.6691(4)$ & $0.4032(3)$ & $0.52507(12)$ & $0.0265(6)$ \\
\hline C18 & $0.5885(5)$ & $0.4885(4)$ & $0.56287(14)$ & $0.0373(8)$ \\
\hline C19 & $0.7239(3)$ & $0.5012(3)$ & $0.48612(12)$ & $0.0244(6)$ \\
\hline C20 & $0.6375(4)$ & $0.5432(4)$ & $0.44793(14)$ & $0.0300(7)$ \\
\hline $\mathrm{C} 21$ & $0.6887(4)$ & $0.6343(4)$ & $0.41261(15)$ & $0.0360(8)$ \\
\hline $\mathrm{C} 22$ & $0.8259(5)$ & $0.6834(4)$ & $0.41507(17)$ & $0.0411(10)$ \\
\hline C23 & $0.9113(5)$ & $0.6426(5)$ & $0.45271(17)$ & $0.0436(10)$ \\
\hline $\mathrm{C} 24$ & $0.8599(4)$ & $0.5528(4)$ & $0.48808(15)$ & $0.0346(8)$ \\
\hline $\mathrm{C} 25$ & $0.2183(5)$ & $0.1311(5)$ & $0.44775(15)$ & $0.0370(9)$ \\
\hline $\mathrm{C} 26$ & $0.2550(4)$ & $0.4057(4)$ & $0.51156(19)$ & $0.0359(7)$ \\
\hline
\end{tabular}




\section{Table S9 Anisotropic Displacement Parameters $\left(\AA^{2}\right)$ for $\left(R_{\mathrm{c}}, \boldsymbol{R}_{H f}\right)-1$. The Anisotropic displacement factor exponent takes the form: - $2 \pi^{2}\left[h^{2} a^{* 2} U_{11}+2 h k a * b^{*} U_{12}+\ldots\right]$.}

\begin{tabular}{|c|c|c|c|c|c|c|}
\hline Atom & $\mathbf{U}_{11}$ & $\mathrm{U}_{22}$ & $\mathrm{U}_{33}$ & $\mathbf{U}_{23}$ & $\mathrm{U}_{13}$ & $\mathbf{U}_{12}$ \\
\hline Hf1 & $0.02290(6)$ & $.01896(6)$ & $0.01935(5)$ & $0.00091(5)$ & $0.00073(5)$ & $0.00307(4)$ \\
\hline N1 & $0.0295(15)$ & $.0237(14)$ & $5(14)$ & $0.0082(10)$ & $.0073(11)$ & $-0.0046(11)$ \\
\hline N2 & $0.0274(12)$ & (10) & 1) & $-0.0022(12)$ & $.0035(12)$ & $-0.0026(9)$ \\
\hline $\mathrm{C} 1$ & 0.024 & $0.041(2)$ & (14) & $0.0033(13)$ & $0.0015(12)$ & $-0.0020(14)$ \\
\hline $\mathrm{C} 2$ & $0.0301(16)$ & & & $.0005(10)$ & & $.0053(13)$ \\
\hline $\mathrm{C} 3$ & $0.0288(16)$ & & & 1) & $.0004(11)$ & $.0038(13)$ \\
\hline$T$ & $0.043(2)$ & 0191(15) & $0275(15)$ & $.0051(11)$ & 4) & $.0055(14)$ \\
\hline C5 & $0.045(2)$ & 7) & $221(14)$ & $-0.0006(12)$ & $.0043(13)$ & $17(15)$ \\
\hline C6 & $0.0260(19)$ & 0.08 & $0.041(2)$ & $0.010(2)$ & $0.0032(16)$ & $-0.002(2)$ \\
\hline $\mathrm{C7}$ & $0.061(3)$ & & 1 & & & (18) \\
\hline$\angle 0$ & $0.034(2)$ & $.049(2)$ & $.0363(19)$ & 17) & $3(15)$ & $-0.0017(17)$ \\
\hline $\mathrm{C} 9$ & $0.071(3)$ & 2) & (2) & $.0099(17)$ & (2) & $0.026(2)$ \\
\hline $\mathrm{C} 10$ & $0.088(4)$ & 2) & (2) & $-0.0001(16)$ & $-0.005(2)$ & $-0.035(2)$ \\
\hline $\mathrm{C} 11$ & 0.028 & & & 0.00 & 0.00 & (11) \\
\hline $\mathrm{C} 12$ & 5) & & & 12) & 12) & (12) \\
\hline $\mathrm{C} 13$ & $0.0300(17)$ & 8) & (16) & 0.00 & 3) & $20(14)$ \\
\hline C14 & $0.042(2)$ & $0355(19)$ & $.0294(17)$ & $-0.0036(14)$ & $0.0119(14)$ & $-0.0014(16)$ \\
\hline $\mathrm{C} 15$ & $0.042(2)$ & $0.0352(19)$ & $0.0263(15)$ & $-0.0083(13)$ & $0.0057(14)$ & $-0.0040(15)$ \\
\hline C16 & $0.0369(1$ & (16) & $0.0319(17)$ & -0.00 & $0.0092(13)$ & 7(14) \\
\hline $\mathrm{C} 17$ & 0.0 & & & -0 & 2) & $2(12)$ \\
\hline C18 & $0.051(2)$ & $0.0270(18)$ & $3(18)$ & $-0.0104(14)$ & $0.0072(16)$ & $-0.0042(16)$ \\
\hline C19 & $0.0255(15)$ & $0.0166(13)$ & $0.0312(15)$ & $-0.0044(11)$ & $0.0000(12)$ & $-0.0015(11)$ \\
\hline $\mathrm{C} 20$ & $0.0290(17)$ & $0.0250(16)$ & $0.0360(17)$ & $0.0016(13)$ & $-0.0014(13)$ & $-0.0020(13)$ \\
\hline $\mathrm{C} 21$ & $0.046(2)$ & 0.02 & 19) & 0.00 & 0.00 & $\partial(16)$ \\
\hline $\mathrm{C} 22$ & $0.053(2)$ & & & $-0.0056(15)$ & $0.0216(19)$ & $54(17)$ \\
\hline $\mathrm{C} 23$ & $0.038(2)$ & $0.042(2)$ & $0.051(2)$ & $-0.0133(18)$ & $0.0139(18)$ & $-0.0144(19)$ \\
\hline $\mathrm{C} 24$ & $0.0285(18)$ & $0.040(2)$ & $0.0356(17)$ & $-0.0092(15)$ & $0.0003(14)$ & $-0.0054(15$ \\
\hline $\mathrm{C} 25$ & $0.039(2)$ & $0.041(2)$ & $0.0313(18)$ & $0.0008(15)$ & $-0.0103(15)$ & $0.0050(17)$ \\
\hline $\mathrm{C} 26$ & $0.0329(17)$ & $0.0318(16)$ & $0.0431(18)$ & $0.0023(19)$ & $0.0005(19)$ & $0.0097(3)$ \\
\hline
\end{tabular}


Table S10 Bond Lengths for $\left(\boldsymbol{R}_{\mathrm{c}}, \boldsymbol{R}_{H f}\right)-1$.

\begin{tabular}{llllll}
\multicolumn{2}{c}{ Atom Atom Length/A } & \multicolumn{3}{l}{ Atom Atom Length/A } \\
$\mathrm{Hf1}$ & $\mathrm{N} 10$ & $2.212(3)$ & $\mathrm{C} 18$ & $\mathrm{C} 20$ & $1.520(5)$ \\
$\mathrm{H} 11$ & $\mathrm{C} 1$ & $2.253(4)$ & $\mathrm{C} 18$ & $\mathrm{C} 19$ & $1.529(5)$ \\
$\mathrm{Hf} 1$ & $\mathrm{~N} 17$ & $2.262(3)$ & $\mathrm{C} 20$ & $\mathrm{C} 25$ & $1.384(5)$ \\
$\mathrm{Hf1}$ & $\mathrm{C} 2$ & $2.277(3)$ & $\mathrm{C} 20$ & $\mathrm{C} 21$ & $1.396(5)$ \\
$\mathrm{Hf1}$ & $\mathrm{C} 30$ & $2.503(3)$ & $\mathrm{C} 21$ & $\mathrm{C} 22$ & $1.393(5)$ \\
$\mathrm{H} 1$ & $\mathrm{C} 31$ & $2.505(3)$ & $\mathrm{C} 22$ & $\mathrm{C} 23$ & $1.383(6)$ \\
$\mathrm{H} 11$ & $\mathrm{C} 32$ & $2.519(3)$ & $\mathrm{C} 23$ & $\mathrm{C} 24$ & $1.381(7)$ \\
$\mathrm{H} 1$ & $\mathrm{C} 34$ & $2.520(4)$ & $\mathrm{C} 24$ & $\mathrm{C} 25$ & $1.386(6)$ \\
$\mathrm{H} 1$ & $\mathrm{C} 33$ & $2.524(4)$ & $\mathrm{C} 30$ & $\mathrm{C} 31$ & $1.412(5)$ \\
$\mathrm{N} 10$ & $\mathrm{C} 16$ & $1.324(4)$ & $\mathrm{C} 30$ & $\mathrm{C} 34$ & $1.415(6)$ \\
$\mathrm{N} 10$ & $\mathrm{C} 11$ & $1.455(4)$ & $\mathrm{C} 30$ & $\mathrm{C} 35$ & $1.505(5)$ \\
$\mathrm{C} 11$ & $\mathrm{C} 12$ & $1.527(5)$ & $\mathrm{C} 31$ & $\mathrm{C} 32$ & $1.416(5)$ \\
$\mathrm{C} 12$ & $\mathrm{C} 13$ & $1.523(6)$ & $\mathrm{C} 31$ & $\mathrm{C} 36$ & $1.510(5)$ \\
$\mathrm{C} 13$ & $\mathrm{C} 14$ & $1.527(6)$ & $\mathrm{C} 32$ & $\mathrm{C} 33$ & $1.425(5)$ \\
$\mathrm{C} 14$ & $\mathrm{C} 15$ & $1.535(5)$ & $\mathrm{C} 32$ & $\mathrm{C} 37$ & $1.501(5)$ \\
$\mathrm{C} 15$ & $\mathrm{C} 16$ & $1.512(5)$ & $\mathrm{C} 33$ & $\mathrm{C} 34$ & $1.423(6)$ \\
$\mathrm{C} 16$ & $\mathrm{~N} 17$ & $1.336(4)$ & $\mathrm{C} 33$ & $\mathrm{C} 38$ & $1.502(6)$ \\
$\mathrm{N} 17$ & $\mathrm{C} 18$ & $1.469(4)$ & $\mathrm{C} 34$ & $\mathrm{C} 39$ & $1.502(5)$
\end{tabular}

Table S11 Bond Angles for $\left(\boldsymbol{R}_{\mathrm{c}}, \boldsymbol{R}_{H f}\right)-1$.

\begin{tabular}{|c|c|c|c|c|c|c|c|}
\hline Ator & Atc & tom & Angle $/^{\circ}$ & to & 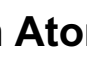 & tom & Angle $/^{\circ}$ \\
\hline N1 & $\mathrm{Hf} 1$ & $\mathrm{C} 25$ & $84.23(14)$ & $\mathrm{C} 2$ & $\mathrm{C} 1$ & $\mathrm{C} 6$ & $125.2(4)$ \\
\hline $\mathrm{N} 1$ & $\mathrm{Hf1}$ & N2 & $58.97(10)$ & C5 & $\mathrm{C} 1$ & C6 & 127.1(4) \\
\hline $\mathrm{C} 25$ & $\mathrm{Hf} 1$ & N2 & $129.33(15)$ & $\mathrm{C} 2$ & $\mathrm{C} 1$ & $\mathrm{Hf} 1$ & $73.43(19)$ \\
\hline N1 & $\mathrm{Hf} 1$ & $\mathrm{C} 26$ & $132.47(14)$ & C5 & $\mathrm{C} 1$ & $\mathrm{Hf} 1$ & $73.4(2)$ \\
\hline 25 & $\mathrm{Hf} 1$ & C26 & $88.94(16)$ & C6 & $\mathrm{C} 1$ & $\mathrm{Hf} 1$ & $121.1(3)$ \\
\hline $\mathrm{N} 2$ & $\mathrm{Hf1}$ & C26 & $92.0 \mathrm{C}$ & C3 & $\mathrm{C} 2$ & C1 & 107.7(3) \\
\hline V1 & $\mathrm{Hf} 1$ & $\mathrm{C} 4$ & 84.48 & C3 & $\mathrm{C} 2$ & $\mathrm{C} 7$ & $126.4(3)$ \\
\hline $\mathrm{C} 25$ & $\mathrm{Hf} 1$ & C4 & $108.34(14)$ & $\mathrm{C} 1$ & $\mathrm{C} 2$ & $\mathrm{C} 7$ & $125.6(3)$ \\
\hline $\mathrm{N} 2$ & $\mathrm{Hf1}$ & C4 & $101.82(12)$ & C3 & $\mathrm{C} 2$ & $\mathrm{Hf} 1$ & $73.00(17)$ \\
\hline $\mathrm{C} 26$ & $\mathrm{Hf} 1$ & $\mathrm{C} 4$ & $141.46(15)$ & $\mathrm{C} 1$ & $\mathrm{C} 2$ & $\mathrm{Hf} 1$ & $73.81(19)$ \\
\hline N1 & $\mathrm{Hf1}$ & $\mathrm{C} 3$ & $101.95(12)$ & $\mathrm{C7}$ & $\mathrm{C} 2$ & $\mathrm{Hf} 1$ & $123.4(2)$ \\
\hline
\end{tabular}




\begin{tabular}{|c|c|c|c|c|c|c|c|}
\hline 25 & ff1 & 3 & 14) & $\mathrm{C} 4$ & C3 & $\mathrm{C} 2$ & \\
\hline o & ff1 & 3 & $7.27(12)$ & $\mathrm{C} 4$ & C3 & co & $125.6(3)$ \\
\hline 26 & If1 & 3 & 6) & $\mathrm{C} 2$ & C3 & 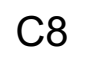 & \\
\hline 4 & If1 & 3 & 2.84( & C4 & 3 & & (19) \\
\hline & f1 & & 2) & $\mathrm{C} 2$ & 3 & f1 & 18) \\
\hline 25 & ff1 & 5 & 3.13( & $\mathrm{C} 8$ & C3 & $\mathrm{f} 1$ & $24.4(2)$ \\
\hline 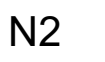 & If1 & 5 & 2) & C5 & $\mathrm{C} 4$ & 3 & $08.0(3)$ \\
\hline 26 & $\mathrm{f} 1$ & 5 & 4) & C5 & $\mathrm{C} 4$ & 9 & 4) \\
\hline 4 & If1 & 5 & $2.57(13)$ & C3 & C4 & C9 & $24.4(4)$ \\
\hline 3 & $\mathrm{f} 1$ & 5 & 4.08 & C5 & $\mathrm{C} 4$ & $\mathrm{Hf} 1$ & 4.4(2) \\
\hline 1 & If1 & 2 & 1) & C3 & C4 & $\mathrm{Hf1}$ & $3.70(1$ \\
\hline 25 & f1 & $\mathrm{C} 2$ & 23.5 & C9 & $\mathrm{C} 4$ & f1 & $23.5(2)$ \\
\hline & ff1 & 5 & & $\mathrm{C} 4$ & & & \\
\hline 26 & f1 & 2 & 7.16 & C4 & C5 & 10 & $125.2(4)$ \\
\hline & f1 & 2 & .4 & C1 & C5 & 10 & (4) \\
\hline 3 & f1 & 2 & 2.8 & $\mathrm{C} 4$ & 5 & $f 1$ & 3.0 \\
\hline 5 & ff1 & $\mathrm{C} 2$ & 4.22 & C1 & $\mathrm{C} 5$ & $f 1$ & 3.8( \\
\hline & 1 & C1 & & $c$ & C5 & ff1 & 3) \\
\hline 25 & ff1 & 1 & ) & 11 & C11 & 2 & 3) \\
\hline 2 & f1 & $\mathrm{C} 1$ & 2) & N1 & & 2 & 3) \\
\hline 26 & f1 & 1 & 22 & 2 & 1 & 12 & 3) \\
\hline & ff1 & C1 & 4.3 & N1 & 1 & $f 1$ & (17) \\
\hline & $\mathrm{Hf} 1$ & 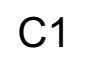 & .2 & N2 & $\mathrm{C} 1{ }^{\prime}$ & $\mathrm{Hf} 1$ & 57.1 \\
\hline 5 & f1 & 1 & .7 & C12 & 11 & f1 & $75.5(2)$ \\
\hline 2 & $\mathrm{f} 1$ & & 2.7 & 1 & 0.12 & C13 & $112.3(3)$ \\
\hline 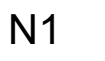 & f1 & 1 & 934 & 4 & 13 & 12 & 15.1(3) \\
\hline 25 & 1 & & . & C15 & 14 & 13 & $5.9(3)$ \\
\hline & $\mathrm{Hf} 1$ & & 29.68 & C16 & 15 & 14 & $13.9(3)$ \\
\hline 26 & $\mathrm{Hf} 1$ & 1 & 1151 & N1 & 16 & 15 & 13.7(3) \\
\hline & $\mathrm{f} 1$ & C11 & 92.2 & 2 & 17 & C19 & $113.5(3)$ \\
\hline 3 & $\mathrm{Hf} 1$ & C11 & 94.03 & N2 & C17 & C18 & $109.6(3)$ \\
\hline C5 & $\mathrm{Hf} 1$ & C11 & 120.4 & C19 & C17 & C18 & 109.5(3) \\
\hline & 1 & C11 & 123.8 & C24 & C19 & $\mathrm{C} 20$ & 118.5(3) \\
\hline C1 & $\mathrm{Hf} 1$ & C11 & $145.73(11)$ & $\mathrm{C} 24$ & C19 & 17 & $120.6(3)$ \\
\hline C11 & 1 & C16 & $122.0(3)$ & C20 & C19 & C17 & $120.9(3)$ \\
\hline & 11 & & $95.8(2)$ & C21 & $\mathrm{C} 20$ & C19 & $120.3(3)$ \\
\hline C16 & N1 & $\mathrm{Hf} 1$ & $141.9(2)$ & $\mathrm{C} 22$ & $\mathrm{C} 21$ & $\mathrm{C} 20$ & $120.1(4)$ \\
\hline C11 & $\mathrm{N} 2$ & C17 & 121.2(3) & C23 & $\mathrm{C} 22$ & C21 & $119.7(4)$ \\
\hline
\end{tabular}


C11 N2 Hf1 93.16(19) C22 C23 C24 120.1(4)

$\begin{array}{llllllll}\mathrm{C} 17 & \mathrm{~N} 2 & \mathrm{H} f 1 & 145.6(2) & \mathrm{C} 19 & \mathrm{C} 24 & \mathrm{C} 23 & 121.2(4)\end{array}$

C2 $\quad$ C1 C5 $107.6(3)$

Table S12 Torsion Angles for $\left(R_{\mathrm{c}}, \boldsymbol{R}_{H f}\right)-1$.
$\begin{array}{llllllllll} & \text { A } & \text { C } & \text { D } & \text { Angle } l^{\circ} & \text { A } & \text { B } & \text { C } & \text { D } & \text { Angle } l^{\circ}\end{array}$

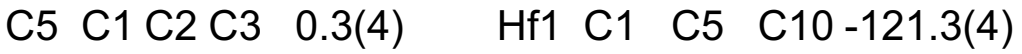

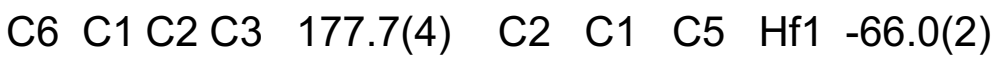

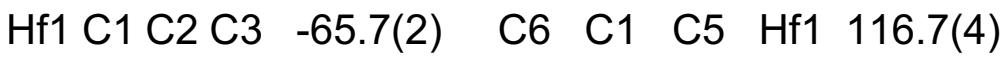
C5 $\mathrm{C} 1 \mathrm{C} 2 \mathrm{C} 7-174.3(3) \quad \mathrm{C} 16 \mathrm{~N} 1 \quad \mathrm{C} 11 \mathrm{~N} 2 \quad-179.5(3)$
$\begin{array}{lllllll}\mathrm{C} 6 & \mathrm{C} 1 \mathrm{C} 2 \mathrm{C} 7 & 3.1(6) \quad \mathrm{Hf} 1 \mathrm{~N} 1 & \mathrm{C} 11 \mathrm{~N} 2 & -4.2(3)\end{array}$
Hf1 C1 C2 C7 119.8(3) C16 N1 C11 C12 0.8(5)
C5 C1 C2 Hf1 66.0(2) Hf1 N1 C11 C12 176.1(3)
C6 C1 C2 Hf1 $-116.7(4) \quad$ C16 N1 C11 Hf1 $-175.3(4)$
$\begin{array}{lllllll}\text { C1 } & \mathrm{C} 2 \mathrm{C} 3 \mathrm{C} 4 & 0.1(4) & \mathrm{C} 17 \mathrm{~N} 2 & \mathrm{C} 11 \mathrm{~N} 1 & -177.7(3)\end{array}$
C7 C2 C3 C4 $174.7(3)$ Hf1 N2 C11 N1 $4.1(3)$
$\mathrm{Hf} 1 \mathrm{C} 2 \mathrm{C} 3 \mathrm{C} 4 \quad-66.1(2) \quad \mathrm{C} 17 \mathrm{~N} 2 \quad \mathrm{C} 11 \mathrm{C} 122.0(5)$
C1 C2 C3 C8 $-172.5(3)$ Hf1 N2 C11 C12 -176.2(3)
C7 C2 C3 C8 2.1(5) C17 N2 C11 Hf1 178.2(4)
Hf1 C2 C3 C8 121.3(3) N1 C11 C12 C13 65.2(4)
C1 C2 C3 Hf1 66.2(2) N2 C11 C12 C13 -114.5(4)
C7 C2 C3 Hf1 -119.3(3) C11 C12 C13 C14 -77.6(4)
C2 C3 C4 C5 -0.5(4) C12 C13 C14 C15 59.1(5)
C8 C3 C4 C5 172.1(3) C13 C14 C15 C16-58.5(5)
Hf1 C3 C4 C5 -67.1(2) C11 N1 C16 C15 -68.2(5)
C2 C3 C4 C9 $-173.6(3)$ Hf1 N1 C16 C15 119.3(4)
C8 C3 C4 C9 -1.0(6) C14 C15 C16 N1 79.8(4)
Hf1 C3 C4 C9 119.8(3) C11 N2 C17 C19 63.2(4)
C2 $\mathrm{C} 3 \mathrm{C} 4 \mathrm{Hf} 1$ 66.6(2) Hf1 N2 C17 C19-119.9(4)
C8 C3 C4 Hf1 -120.8(3) C11 N2 C17 C18 -174.0(3)
C3 $\mathrm{C} 4 \mathrm{C} 5 \mathrm{C} 1 \quad 0.7(4) \quad \mathrm{Hf} 1 \mathrm{~N} 2 \quad \mathrm{C} 17 \mathrm{C} 182.9(6)$
C9 C4 C5 C1 173.5(3) N2 C17 C19 C24 -141.3(3)
Hf1 C4 C5 C1 -65.9(3) C18 C17 C19 C24 95.8(4)
C3 C4 C5 C10 -172.7(3) N2 C17 C19 C20 39.7(4) 

C9 C4 C5 C10 0.1(6) C18 C17 C19 C20 -83.2(4)
Hf1 C4 C5 C10 120.7(4) C24 C19 C20 C21 0.4(5)
C3 C4 C5 Hf1 66.6(2) C17 C19 C20 C21 179.4(3)
C9 C4 C5 Hf1 -120.6(4) C19 C20 C21 C22 0.3(6)
C2 C1 C5 C4 -0.7(4) C20 C21 C22 C23 -0.4(6)
C6 C1 C5 C4 -177.9(4) C21 C22 C23 C24 -0.1(6)
Hf1 C1 C5 C4 65.3(2) C20 C19 C24 C23 -1.0(5)
C2 C1 C5 C10 172.7(3) C17 C19 C24 C23 -180.0(3)
C6 C1 C5 C10 -4.6(6) C22 C23 C24 C19 0.8(6)

Table S13 Hydrogen Atom Coordinates and Isotropic Displacement Parameters $\left(\AA^{2}\right)$ for $\left(R_{\mathrm{c}}, R_{H f}\right)$-1.

\begin{tabular}{lllll} 
Atom & \multicolumn{1}{c}{$\boldsymbol{x}$} & \multicolumn{1}{c}{$\boldsymbol{y}$} & \multicolumn{1}{c}{$\boldsymbol{z}$} & \multicolumn{1}{c}{$\mathbf{U}(\mathbf{e q})$} \\
H6A & -0.00332 & 0.085793 & 0.539114 & 0.077 \\
H6B & 0.026741 & 0.226986 & 0.56893 & 0.077 \\
H6C & -0.00822 & 0.083516 & 0.596782 & 0.077 \\
H7A & 0.243612 & 0.25186 & 0.661431 & 0.061 \\
H7B & 0.19226 & 0.34368 & 0.616341 & 0.061 \\
H7C & 0.354693 & 0.344613 & 0.631818 & 0.061 \\
H8A & 0.576777 & 0.06045 & 0.64232 & 0.059 \\
H8B & 0.557996 & 0.223554 & 0.629175 & 0.059 \\
H8C & 0.642301 & 0.121506 & 0.593434 & 0.059 \\
H9A & 0.607034 & -0.086187 & 0.543559 & 0.074 \\
H9B & 0.4898 & -0.180955 & 0.51771 & 0.074 \\
H9C & 0.521067 & -0.200759 & 0.574001 & 0.074 \\
H10A & 0.159926 & -0.216918 & 0.543092 & 0.083 \\
H10B & 0.255832 & -0.184231 & 0.496973 & 0.083 \\
H10C & 0.107416 & -0.106429 & 0.503384 & 0.083 \\
H12A & 0.815326 & 0.086491 & 0.465811 & 0.032 \\
H12B & 0.83786 & 0.251076 & 0.475734 & 0.032 \\
H13A & 0.732253 & 0.290969 & 0.395647 & 0.037 \\
H13B & 0.889822 & 0.232023 & 0.396375 & 0.037 \\
H14A & 0.759434 & 0.112352 & 0.337911 & 0.043 \\
H14B & 0.809531 & 0.004307 & 0.378528 & 0.043 \\
H15A & 0.529746 & 0.111843 & 0.369003 & 0.041 \\
H15B & 0.57719 & -0.045171 & 0.355944 & 0.041
\end{tabular}




$\begin{array}{lllll}\text { H16A } & 0.475383 & -0.075285 & 0.42821 & 0.037 \\ \text { H16B } & 0.63857 & -0.068076 & 0.442274 & 0.037 \\ \text { H1 } & 0.752051 & 0.358654 & 0.541266 & 0.032 \\ \text { H18A } & 0.505559 & 0.531777 & 0.547911 & 0.056 \\ \text { H18B } & 0.649843 & 0.56231 & 0.575881 & 0.056 \\ \text { H18C } & 0.558366 & 0.425991 & 0.589048 & 0.056 \\ \text { H20 } & 0.543437 & 0.50943 & 0.446025 & 0.036 \\ \text { H21 } & 0.62934 & 0.662696 & 0.386807 & 0.043 \\ \text { H22 } & 0.860924 & 0.745001 & 0.390885 & 0.049 \\ \text { H23 } & 1.005509 & 0.675945 & 0.454467 & 0.052 \\ \text { H24 } & 0.919325 & 0.526367 & 0.514116 & 0.042 \\ \text { H25A } & 0.237104 & 0.191948 & 0.419943 & 0.055 \\ \text { H25B } & 0.119997 & 0.142447 & 0.457842 & 0.055 \\ \text { H25C } & 0.235209 & 0.032612 & 0.438809 & 0.055 \\ \text { H26A } & 0.275755 & 0.451251 & 0.542467 & 0.054 \\ \text { H26B } & 0.152929 & 0.39372 & 0.508187 & 0.054 \\ \text { H26C } & 0.290336 & 0.464605 & 0.485167 & 0.054\end{array}$

\section{$\underline{\text { References }}$}

1. Schock, L. E.; Marks, T. J. Organometallic thermochemistry. Metal hydrocarbyl, hydride, halide, carbonyl, amide, and alkoxide bond enthalpy relationships and their implications in pentamethylcyclopentadienyl and cyclopentadienyl complexes of zirconium and hafnium. J. Am. Chem. Soc. 1988, 110, 7701-7715.

2. Grisar, J. M.; Claxton, G. P.; MacKenzie, R. D. Substituted a-methylbenzyl and tricyclic arylalkyl lactamimides as inhibitors of blood platelet aggregation. J. Med. Chem. 1976, 19, 503-508. 